\title{
Gelfand-type duality for commutative von Neumann algebras
}

\section{Dmitri Pavlov}

\author{
Department of Mathematics and Statistics, Texas Tech University \\ https://dmitripavlov.org/
}

\begin{abstract}
We show that the following five categories are equivalent: (1) the opposite category of commutative von Neumann algebras; (2) compact strictly localizable enhanced measurable spaces; (3) measurable locales; (4) hyperstonean locales; (5) hyperstonean spaces. This result can be seen as a measure-theoretic counterpart of the Gelfand duality between commutative unital $\mathrm{C}^{*}$-algebras and compact Hausdorff topological spaces. This paper is also available as arXiv:2005.05284v3.
\end{abstract}

\section{Contents}

1. Introduction

1.3. Structure of the paper

1.4. Prerequisites

1.5. Conventions

1.7. Future directions

1.8. Fiberwise measures and disintegrations

1.10. Bundles of Hilbert spaces and topological vector spaces

1.12. The setting of arbitrary elementary toposes

1.13. Acknowledgments

2. Stone-type dualities for locales

2.1. Review of locales

2.18. Valuations on locales

2.23. Stone duality for distributive lattices

2.33. Stone duality for Boolean algebras

2.42. Stonean duality

2.51. Hyperstonean duality

3. Duality between measurable locales and commutative von Neumann algebras

3.1. Commutative von Neumann algebras

3.5. Functors between commutative von Neumann algebras and measurable locales

3.15. Equivalence of commutative von Neumann algebras and measurable locales

4. Point-set measurable spaces

4.1. Enhanced measurable spaces

4.10. Equality almost everywhere

4.19. Comparison of equivalence relations on morphisms

4.24. Coproducts of enhanced measurable spaces

4.27. Measures on enhanced measurable spaces

4.33. Localizable enhanced measurable spaces

4.37. Essential measures on enhanced measurable spaces

4.47. Strictly localizable enhanced measurable spaces

4.56. Compact enhanced measurable spaces

5. Equivalence between measurable spaces and measurable locales

5.1. From hyperstonean spaces to enhanced measurable spaces

5.7. From enhanced measurable spaces to measurable locales

5.13. Equivalence of compact strictly localizable enhanced measurable spaces and measurable locales

6. References 


\section{Introduction}

In 1939, Israel Gelfand 1941] established a duality between compact Hausdorff topological spaces and commutative unital $\mathrm{C}^{*}$-algebras. This duality can be formulated (Negrepontis [1971.a]) as a contravariant equivalence of categories, where one takes continuous maps of topological spaces and unital $\mathrm{C}^{*}$-homomorphisms of $\mathrm{C}^{*}$-algebras respectively as morphisms.

Based on this, one can conjecture an analogous duality between an appropriate variant of measurable spaces and commutative von Neumann algebras. It is fairly easy to guess some ingredients for functors going in both directions for such a duality. Given a measurable space $X$, one can construct a commutative complex *-algebra of bounded complex measurable functions on $X$. If $X$ is equipped with a $\sigma$-ideal of negligible sets (for example, induced from some measure by taking its sets of measure 0), we can define the relation of equality almost everywhere and take the quotient of the above algebra by this equivalence relation. Under additional assumptions, such as $\sigma$-finiteness or, more generally, localizability, this *-algebra is a von Neumann algebra. A $\sigma$-ideal of negligible sets is necessary for the above construction, so we can expect it to be present in some form in the statement of the duality. Thus, we consider triples $(X, M, N)$, where $X$ is a set, $M$ is a $\sigma$-algebra of measurable subset of $X$, and $N \subset M$ is a $\sigma$-ideal of negligible subsets of $X$. We refer to such a triple as an enhanced measurable space (Definition 4.4).

Conversely, given a commutative von Neumann algebra, one can take the Gelfand spectrum of its underlying $\mathrm{C}^{*}$-algebra or, equivalently, the Stone spectrum 1937.a of its Boolean algebra of projections. The resulting topological spaces are known as hyperstonean spaces (Definition 2.66). They were introduced and studied by Dixmier 1951.a]. Dixmier [1951.a, Théorème 2] also proved that hyperstonean spaces are precisely the Gelfand spectra of commutative von Neumann algebras. Any hyperstonean space can be equipped with a $\sigma$-ideal of negligible sets comprising precisely the meager subsets (as already proposed by Loomis [1947] and Sikorski $|\mathbf{1 9 4 8}|$ ) and a $\sigma$-algebra of measurable subsets comprising precisely the symmetric differences of open and meager subsets. This produces an enhanced measurable space (Lemma 5.3). Only a $\sigma$-ideal of negligible sets can be defined canonically in this construction, not a specific measure. Concerning morphisms of commutative von Neumann algebras, von Neumann [1932], C. Ionescu Tulcea [1965, Vesterstrøm-Wils 1969|, Edgar |1976|, Graf [1980|, as well as von Neumann-Maharam |1958] established criteria for lifting homomorphisms of Boolean algebras or commutative von Neumann algebras to point-set measurable maps of measurable spaces.

However, a formulation that promotes these constructions to an actual equivalence of categories does not appear in the literature. In particular, whereas on the von Neumann algebra side it is clear that one should take the category of commutative von Neumann algebras and normal *-homomorphisms, on the measure theory side the situation is far from clear. Some obvious choices for objects and morphisms, such as localizable enhanced measurable spaces with morphisms being equivalence classes of measurable maps modulo equality almost everywhere, fail to produce a category that is contravariantly equivalent to the category of commutative von Neumann algebras.

This paper resolves these issues by establishing the following result.

Theorem 1.1. The following categories are equivalent.

- The category CSLEMS of compact strictly localizable enhanced measurable spaces (Definition 4.59), whose objects are triples $(X, M, N)$, where $X$ is a set, $M$ is a $\sigma$-algebra of measurable subsets of $X$, $N \subset M$ is a $\sigma$-ideal of negligible subsets of $X$ such that the additional conditions of compactness (Definition 4.57) and strict localizability (Definition 4.48) are satisfied. Morphisms $(X, M, N) \rightarrow\left(X^{\prime}, M^{\prime}, N^{\prime}\right)$ are equivalence classes of maps of sets $f: X \rightarrow X^{\prime}$ such that $\left(f^{*}\right) ! M^{\prime} \subset M$ and $\left(f^{*}\right) ! N^{\prime} \subset N$ (superscript $*$ denotes the preimage map, subscript ! denotes the direct image map) modulo the equivalence relation of weak equality almost everywher (Definition 4.13): $f \approx g$ if for all $m \in M^{\prime}$ the symmetric difference $f^{*} m \oplus g^{*} m$ belongs to $N$.

- The category HStonean of hyperstonean spaces and open maps (Definition 2.66).

- The category HStoneanLoo of hyperstonean locales and open maps (Definition 2.61).

- The category MLoc of measurable locales, defined as the full subcategory of the category of locales consisting of complete Boolean algebras that admit sufficiently many continuous valuations (Definition 2.55).

- The opposite category CVNA of of commutative von Neumann algebras, whose morphisms are norma *-homomorphisms of algebras in the opposite direction (Definition 3.3). 
Furthermore, as explained in the paper, the equivalences are implemented by the following four adjoint equivalences, providing a highly structured way to move between these five different settings:

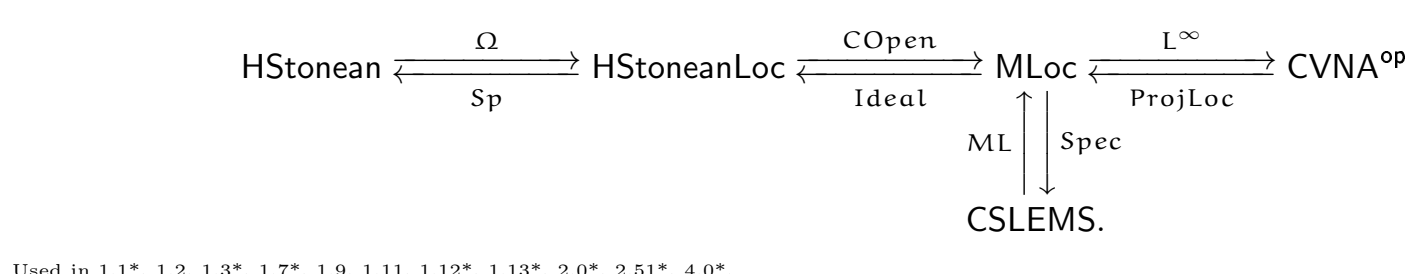

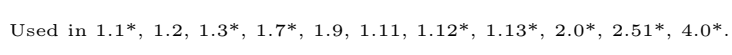

Proof. Combine Proposition 2.72, Proposition 2.73, Theorem 3.20, and Theorem 5.19. I

The last four categories appear to be rather natural, but the first category CSLEMS may appear unfamiliar, both in terms of its objects and morphisms. We offer some clarifying remarks that may help to convince the reader that CSLEMS is a natural and essentially the only possible choice here.

First, the category CSLEMS is sensitive to the choices like localizability versus strict localizability, the presence of compactness property, or equality almost everywhere versus weak equality almost everywhere. Only the choices made in Theorem 1.1 produce a category contravariantly equivalent to commutative von Neumann algebras. In particular, the equivalence relation of weak equality almost everywhere differs from the equivalence relation of equality almost everywhere if the $\sigma$-algebra is not countably separated (Definition 4.21). See Lemma 4.22 and Remark 4.23.

The requirement that $N$ is a $\sigma$-ideal of $2^{X}$ (as opposed to a $\sigma$-ideal of $M$ ) amounts to incorporating the completeness assumption in our definition of an enhanced measurable space. The completeness condition does not change the resulting category, but considerably simplifies the presentation, since in this case we can define morphisms of enhanced measurable spaces as equivalence classes of point-set maps whose associated preimage map preserves measurable and negligible subsets. See Remark 4.8, which explains how the noncomplete version works and why it is equivalent to the complete version.

The strict localizability property is a natural generalization of $\sigma$-finiteness that is necessary since we allow non- $\sigma$-finite commutative von Neumann algebras. The compactness property is the abstract measuretheoretic formulation of Radon measures. Indeed, a Radon measure on a topological space yields a compact enhanced measurable space by Example 4.60. As explained there, the resulting space is also strictly localizable, which means that the overwhelming majority of measurable spaces in analysis are in fact compact strictly localizable enhanced measurable spaces. The properties of compactness and strict localizability are also essential for eliminating pathologies that inevitably arise with larger classes of enhanced measurable spaces. For instance, they are crucial for establishing Proposition 4.65, which says that a morphism of enhanced measurable spaces has a well-defined image, which is a measurable set. Another crucial point at which these conditions are relevant is Proposition 5.16, which lies at the core of the equivalence between CSLEMS and MLoq.

The functor Spec $\circ$ ProjLod: CVNA $\rightarrow$ EMS (Definition 5.5, Definition 3.10) produces the Gelfand spectrum of the underlying commutative $\mathrm{C}^{*}$-algebra equipped with a 6 -algebra of measurable subsets and $\sigma$-ideal of negligible subsets, described above as the Loomis-Sikorski construction. By Proposition 5.4, enhanced measurable spaces in the image of this functor are compact and strictly localizable. Furthermore, by Lemma 4.63 5 such spaces are closed under isomorphisms in the category LDEMS of locally determined enhanced measurable spaces (Definition 4.50). This is not obvious, since the property of compactness is formulated in terms of properties of the $\sigma$-algebra and $\sigma$-ideal that are not manifestly invariant under isomorphisms in LDEMS. In particular, Example 4.49 and 1.62 show that strictly localizable enhanced measurable spaces and compact strictly localizable enhanced measurable spaces are not closed under isomorphisms in EMS. The algebra of equivalence classes of bounded complex-valued functions on a localizable enhanced measurable space is a commutative von Neumann algebra, whose Gelfand spectrum is isomorphic to the original enhanced measurable space if the latter is compact and strictly localizable. Thus, the category CSLEMAS of compact strictly localizable enhanced measurable spaces is the essential image of the functor CVNA ${ }^{\circ \mathrm{op}} \rightarrow$ EMS. In other words, the category CSLEMS is forced on us by the nature of this equivalence and we essentially have no other choice.

Larger categorifs like the category LEMS of localizable enhanced measurable spaces, can also be made equivalent to CVNA ${ }^{\text {o }}$ with additional work, but at the cost of losing the convenient point-set description 
of morphisms, since the category of localizable enhanced measurable spaces that is equivalent to CVNA is not LEMS itself, but rather LEMSd, which is obtained from LEMS by discarding certain morphisms and performing a Gabriel-Zisman localization of the resulting nonfull subcategory [LESC with respect to a certain class of morphisms, see Remark 5.18. In the resulting category LEMSd, morphisms are not equivalence classes of maps of sets, but rather abstract compositions of such morphisms and their inverses, which makes LEMSd' less convenient in practice.

Naturally, after Theorem 1.1 one is led to wonder whether the various notions of a measure defined on spaces in these equivalent categories are also equivalent to each other. This is indeed true, although for simplicity and brevity, we formulate the following result for finite measures, even though it continues to hold for infinite measures (semifinite or not).

Theorem 1.2. In the context of the chain of equivalences in Theorem 1.1, we have canonical bijections between the following notions of a complex finite measure:

- Finite essential measures on an enhanced measurable space (Definition 4.40);

- Finite normal measures on a hyperstonean space (Definition 2.62);

- Normal valuations on a hyperstonean locale (Definition 2.57);

- Continuous valuations on a measurable locale (Definition 2.19);

- Elements of the predual of a commutative von Neumann algebra (Definition 3.3).

Used in $1.2^{*}, 1.8 * 1.9$

Proof. Combine Lemma 2.69, Lemma 2.58, Proposition 3.9, and Proposition 4.45. I

The appearance of essential measures is motivated as follows. A finite measure on an enhanced measurable space $(X, M, N)$ is a countably additive map $\mu: M \rightarrow \mathbf{C}$ that vanishes on $N$ (Definition 4.28). Vanishing on $N$ is analogous to absolute continuity. A measure $\mu$ is essential Definition 4.40) if for any $m \in M$ such that $\left.\mu\right|_{m} \neq 0$ we can find a $\sigma$-finite measurable subset $m^{\prime} \subset m$ such that $\left.\mu\right|_{m^{\prime}} \neq 0$. In other words, if an essential measure vanishes on all $\sigma$-finite measurable subsets of some $m \in M$, then it vanishes on all measurable subsets of $m$. Here a measurable subset $m \in M$ is $\sigma$-finite (Definition 4.38) if there is a faithful finite measure $\nu$ on the induced enhanced measurable space $\left(m, M_{m}, N_{m}\right)$ (Definition 4.5), and a measure is faithful if $\left.\nu\right|_{m}=0$ implies $m \in N$.

The condition of essentiality of a measure is nontrivial only in the non- $\sigma$-finite case. Without this condition, even the most fundamental theorems of measure theory, such as the Radon-Nikodym theorem, fail in the non- $\sigma$-finite case, even assuming compactness and trict localizability, see Remark 4.43 and Remark 4.44. If $(X, M, N)$ is localizable (Definition 2.52), i.e., the Boolean algebra $M / N$ is complete, being essential is equivalent to being a completely additive measure (Proposition 4.41). Thus, given Theorem 1.2, we argue that essential measures are the correct notion of a measure in the non- $\sigma$-finite setting.

We conclude with some remarks on the relation between locales and measure theory, as featured in two out of five categories under consideration. An overview of locales is given in $\$ 2.1$, where one can find further references. The category of measurable locales (Definition 2.55) was proposed by the author on December 15, 2010 in 2010.c as a localic analog of localizable enhanced measurable spaces. One passes from a localizable enhanced measurable space $(X, M, N)$ to the corresponding measurable locale by taking the quotient Boolean algebra $M / N$. Boolean algebras of such nature have been studied for a long time: already in 1942, Halmos and von Neumann [1942] proved that the real line as a measurable space can be characterized in terms of its Boolean algebra of equivalence classes of measurable sets. Volume 3 of Fremlin's Measure theory 2002.d] studies localizable measure algebras, which in our context are precisely measurable locales equipped with a possibly infinite positive faithfu] continuous valuation. Jackson 2006, §3.3] observes that sheaves on a $\sigma$-algebra from a localic topos, with the equality almost everywhere defining a Lawvere-Tierney topology, whose sheaves again form a localic topos. Assuming localizability, the resulting locale is isomorphic to the locale $M / N$ discussed above. However, morphisms of measurable spaces are not discussed in this context.

The category of measurable locales MLod is a full subcategory of the category of locales Loc. In particular, morphisms of measurable locales, which correspond to equivalences classes of measurable maps in point-set measure theory, are in bijective correspondence with morphisms of locales, which correspond to continuous maps in point-set general topology. This demonstrates the power of pointfree general topology: it is capable of treating both the traditional general topology and measure theory using the same formalism of locales. 


\subsection{Structure of the paper}

The structure of this paper follows the structure of the chain of functors in Theorem 1.1.

$\$ 2$ explores in the detail the middle part of the chain of equivalences in Theorem 1.1, concerning the categories HStonean, HStoneanLoc, and MLod together with the two adjoint equivalences (Proposition 2.72, Proposition 2.73) relating them. It obtains these two adjunctions by restricting the classical adjoint equivalences between the categories Stone (Stone spaces), StoneLod (Stone locales), and BAlgop (Boolean algebras). As an intermediate step, we pass through the known adjoint equivalences between the categories Stonean (Stonean spaces), StoneanLod (Stonean locales), and CBAlg $\mathrm{op}$ (complete Boolean algebrat).

$\S 3$ constructs the adjoint equivalence (Theorem 3.20) between MLod and [CVNA ${ }^{\mathrm{ob}}$ using arguments resembling the Lebesgue integration theory. Parts of this result appeared in the literature before (e.g., the essential surjectivity of ProjLod follows from the results by Segal [1951.d]), but there does not appear to be a published source that presents a proof of the whole result.

$\$ 4$ carefully defines the appropriate category CSLEMS of point-set measurable spaces and establishes its elementary properties.

$\overline{85}$ constitutes the central core of this paper. It constructs the functors Spec:MLod $\rightarrow$ CSLEMS and ML: CSLEMS $\rightarrow$ MLod and then constructs from them an adjoint equivalence between CSLEMS and MLod.

\subsection{Prerequisites}

We assume familiarity with the elementary theory of categories, functors, natural transformations, adjunctions, equivalences, limits, colimits, and quotient categories. We also assume familiarity with the elementary theory of topological spaces and topological vector spaces. We review the necessary facts about locales in \$2.1 and von Neumann algebras in \$3.1, but our exposition is not self-contained and we give pointers to the literature where appropriate, in particular we cite Johnstone 1982] as much as we can for Stone duality and Sakai 1971.b for several facts about von Neumann algebras. Our presentation of point-set measure theory is self-contained except that we refer to Corollary 341Q, Theorem 343B, and Lemma 451Q in Fremlin 2002.d, 2003 for the lifting theorems and a technical lemma about compact measure spaces, and we also cite several counterexamples from Fremlin.

\subsection{Conventions}

All rings are by definition unital and all homomorphisms of rings by definition preserve units. This applies, in particular, to $\mathrm{C}^{*}$-algebras and their ${ }^{*}$-homomorphisms, which is different from the standard convention.

We use the sans serif font for categories like Top, Set and the Euler font for functors like F, G, etc.

Notation 16. If $C$ is a category, then $C^{\circ}$ denotes its opposite category. Likewise, if $F: C \rightarrow D$ is a functor, then $\mathrm{F}^{\mathrm{op}}: \mathrm{Cop} \rightarrow \mathrm{D} \mathrm{ep}$ is its oppesite functor. Finally, if $t: \mathrm{F} \rightarrow \mathrm{G}$ is a natural transformation of functors

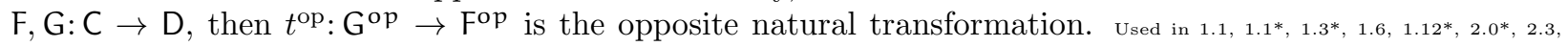

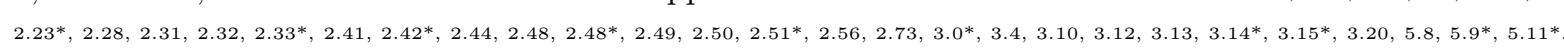

If $f: X \rightarrow Y$ is a map of sets, then $f^{*} B \subset X$ denotes the preimage of $B \subset Y$ and $f ! A \subset Y$ denotes the image of $A \subset X$. The notation $f^{-1}$ is reserved for the inverse of $f$, i.e., the map $f^{-1}: Y \rightarrow X$ such that $f^{-1} \circ f=\operatorname{id}_{X}$ and $f \circ f^{-1}=\operatorname{id}_{Y}$.

We use the terms "meet" and "infimum", as well as "join" and "supremum" interchangeably for any poset. Likewise, we make no distinction between $\bigwedge$ and inf, or between $\bigvee$ and sup.

Hyperlinks are used extensively in this paper, not only for standard items, like numbered statements, bibliography, and back references, including the "Used in" lists at the end of referenced theorems, but also for terms inside the main text, such as "enhanced measurable space" or "hyperstonean locale", as well as identifiers of mathematical objects like "EMS' and "Spec", which are all hyperlinked to their definition.

\subsection{Future directions}

After establishing Theorem 1.1, one almost immediately runs into the question whether various objects that can be defined on measurable spaces, such as measures or measurable fields of Hilbert spaces, also lead to a similar chain of equivalences. This turns out to be true, but considerations of length prevent us from 
proving these results in this paper. The next two subsections briefly sketch the relevant statements. The third subsection explores the setting of elementary toposes.

\subsection{Fiberwise measures and disintegrations}

As explained in Theorem 1.2, each of the five categories introduced above allows for a notion of measure, and all these notions turn out to be equivalent in a precise sense. For applications, it is important to consider relative measures (alias fiberwise measures), conditional expectations in probability theory being an important special case of relative measures. Given a map $f: X \rightarrow Y$, a relative measure $\mu$ on $f$ is roughly a choice of a measure $\mu_{y}$ on $f^{*}\{y\}$ for each point $y \in Y$ so that the family $\left\{\mu_{y}\right\}_{y \in Y}$ is itself in some sense measurable, for example, the map $y \mapsto \mu_{y}\left(m \cap f^{*}\{y\}\right)$ must be measurable for any measurable subset $m \subset X$. If $Y=\{*\}$ is a point, then a relative measure on the unique map $f: X \rightarrow Y$ is simply a measure on $X$ in the usual sense. On the other hand, a relative measure on the identity map id: $X \rightarrow X$ should be a measurable function $X \rightarrow \mathbf{C}$, since a measure on a singleton space is just a number.

If $\mu$ is a relative measure on $f: X \rightarrow Y$ and $\nu$ is a relative measure on $g: Y \rightarrow Z$, then we can construct a relative measure $\nu \circ \mu$ on $g \circ f: X \rightarrow Z$ by assigning to $z \in Z$ the measure $(\nu \circ \mu)_{z}$ on $f^{*} g^{*}\{z\}$ such that

$(\nu \circ \mu)_{z}(m)=\int_{g^{*}\{z\}}\left(y \mapsto \mu_{y}\left(m \cap f^{*}\{y\}\right)\right) \mathrm{d} \nu_{z}$ for any measurable subset $m \subset f^{*} g^{*}\{z\}$. The associativity of composition is established using a generalized Fubini theorem. Thus, we can take any of the above five categories, equip its morphisms with an additional data of a relative measure, and obtain a new category. This allows us to give a rather general statement about equivalence of various notions of relative measures, whose proof will appear elsewhere.

Pretheorem 1.9. In the context of the chain of equivalences in Theorem 1.1, for each of the five categories $C$ in the statement we have a corresponding category $\hat{C}$, which has the same objects and whose morphisms are morphisms in the old sense equipped with an additional fiberwise measure-like structure with individual fibers like in Theorem 1.2 .

The functors in the chain of equivalences in Theorem 1.1 can be promoted to functors between such enhanced categories (so that on the underlying old data they coincide with the previously defined functors), and they remain adjoint equivalences. Restricting to real, positive, and/or finite measures also produces a chain of adjoint equivalences. Finally, the forgetful functors from the above categories to the categories defined in Theorem 1.1 form (contravariant) Grothendieck fibrations.

The last claim about Grothendieck fibrations serves as a really neat formulation of the disintegration theorem of von Neumann, Rohlin, Pachl, and others.

\subsection{Bundles of Hilbert spaces and topological vector spaces}

For each of the five categories introduced above we can define Hilbert bundles, and more generally, bundles of topological vector spaces, and all these notions turn out to be equivalent in a precise sense. We state the following theorem for the case of Hilbert spaces, since this is the more familiar setting.

Pretheorem 1.11. In the context of the chain of equivalences in Theorem 1.1, for each of the five categories $\mathrm{C}$ in the statement we have a corresponding category $\hat{\mathrm{C}}$, whose objects are pairs $(X, V)$, where $X \in \mathrm{C}$ and $V$ is a bundle of Hilbert spaces over $X$. Morphisms $(X, V) \rightarrow\left(X^{\prime}, V^{\prime}\right)$ are pairs $(f, g)$, where $f: X \rightarrow X^{\prime}$ is a morphism in $C$ and $g: V \rightarrow f^{*} V^{\prime}$ is an appropriately defined fiberwise linear map of bundles. The functors in the chain of equivalences in Theorem 1.1 can be promoted to functors between such enhanced categories (so that on the $X$-component they coincide with the previously defined functors), and they remain adjoint equivalences.

The above result, for example, immediately implies the spectral theorem for families of commuting normal operators on a Hilbert space.

The more general version for bundles of topological vector spaces that we did not state above also has many practical applications: it immediately implies the reduction theory for von Neumann algebras, for example. 


\subsection{The setting of arbitrary elementary toposes}

Henry 2014, Theorem 4.2.5] shows that in any elementary topos with a natural numbers object (which generalizes the usual Zermelo-Fraenkel category of sets) one has a contravariant equivalence between the category of commutative localic $\mathrm{C}^{*}$-algebras and the category of compact regular locales.

This improves and strengthens the usual Gelfand duality for $\mathrm{C}^{*}$-algebras by promoting it to arbitrary elementary toposes that need not satisfy the axiom of choice or the law of excluded middle.

As an immediate practical application, one obtains versions of Gelfand duality for smooth or continuous bundles of $\mathrm{C}^{*}$-algebras, as well as equivariant analogs.

Naturally, one is led to wonder to what extent the chain of adjoint equivalences in Theorem 1.1 continues to hold for elementary toposes with a natural number object. Of course, just like in the case of $\mathrm{C}^{*}$-algebras, there is no hope for point-set notions to be equivalent to localic notions due to the lack of the axiom of choice, which is necessary in Proposition 2.27 to show that all coherent locales (in particular, all hyperstonean locales) are spatial, essentially by ensuring the existence of sufficiently many maximal ideals using Zorn's lemma. The axiom of choice is also essential for Proposition 5.16, which is the main ingredient of the equivalence of categories between CSLEMS and MLod The resulting theory makes it clear that the localic notions are the correct ones, so the categories CSLEMS and HStonean must be discarded

Of the remaining three categories HStoneanLod, MLod, and CVNA 9 , the first two are already defined in terms of locales. Of course, instead of merely demanding the existence of sufficiently many hormal valuations respectively continuous valuations one must instead construct the locale of normal valuations respectively continuous valuations in a pointfree manner, and then use it to state the localic analogs of the above properties.

One can also identify some plausible ingredients that are likely to participate in the localic version of the category CVNA of commutative von Neumann algebras. The first ingredient is a complex *-algebra object $A$ in the category of locales (with complex numbers themselves understood as a locale). The underlying locale of $A$ encodes the ultraweak topology on a von Neumann algebra. The second ingredient is a Banach locald $A_{*}$ (defined in Henry [2014, Proposition 4.1.3]) corresponding to the predual equipped with the norm topology. Finally, we should have an isomorphism $A \rightarrow\left(A_{*}\right)^{*}$, where the right side is interpreted as the localic weak-* dual of $A_{*}$ (for the unit ball version see Henry [2014, §4.2.3]).

Whether or not the above categories are actually equivalent in any elementary topos with a natural numbers object is an open problem.

\subsection{Acknowledgments}

I thank the MathOverflow community, where a part of Theorem 1.1 was first stated in [2009], with further developments in [2010.a], 2010.b], and 2010.] proving to be particularly influential in the writing of this paper.

I thank Andre Kornell, whose inquiries about the main theorem in 2012 and 2018 inspired Remark 5.18 and provided an additional impetus to write this paper. I thank Simon Henry for several conversations concerning localic Gelfand duality. I thank Robert Furber for pointing out Lemma 451Q in Fremlin [2003], Remark 5.12, and a missing step in the proof of Lemma 5.11. I thank the anonymous referee of the Journal of Pure and Applied Algebra for suggesting numerous corrections and improvements. 


\section{Stone-type dualities for locales}

This section examines the hyperstonean duality, which is used later to construct enhanced measurable spaces out of measurable locales. The duality is expressed as a chain of adjoint equivalences of categories.

We start with a brief overview of locales and the chain of adjoint equivalences (Proposition 2.27, Proposition 2.31

$$
\text { Coh } \rightleftarrows \text { CohLog } \rightleftarrows \text { DLat }
$$

between coherent spaces, coherent locales, and distributive lattices that serves as a foundation for all Stonetype dualities considered later. The equivalence between Coh and DLat ${ }^{\circ}$ was established by Stone [1938].

Restricting to the full subcategories of Stone spaces, Stone locales, and Boolean algebras yields the classical Stone duality for Boolean algebras (Stone 1936, 1937.a]), expressed via the following chain of adjoint equivalences (Proposition 2.38, Proposition 2.41):

$$
\text { Stone } \rightleftarrows \text { StoneLod } \rightleftarrows \text { BAlgo } 0 \text {. }
$$

Here Stone locales and Stone spaces are defined as compact zero-dimensional locales (respectively, compact zero-dimensional sober topological spaces).

Restricting to (nonfull) subcategories of Stonean spaces, Stonean locales, and complete Boolean algebras produces another chain of adjoint equivalences (Proposition 2.47, Proposition 2.48), known as the Stonean duality (Stone [1937.b]):

$$
\text { Stonean } \rightleftarrows \text { StoneanLog } \rightleftarrows \text { CBAlg }
$$

Here Stonean locales and Stonean spaces are defined as extremally disconnected Stone locales (respectively Stone spaces). CBAlg is the category of complete Boolean algebras with supremum-preserving homomorphisms as morphisms.

Finally, restricting again to full subcategories of hyperstonean space, hyperstonean locales, and localizable Boolean algebras produces the chain of adjoint equivalences from Theorem 1.1 (Proposition 2.72, Proposition 2.73):

$$
\text { HStonean } \rightleftarrows \text { HStoneanLod } \rightleftarrows \text { LBAlg }
$$

\subsection{Review of locales Used in $1.2+1.4$}

For an accessible introduction to locales, see Chapter 1 of Borceux 1994.a.

Definition 2.2. The category of frames Frm is defined as follows. A frame is a poset $L$ that admits finite infima (alias meets, $\wedge$ ), arbitrary suprema (alias joins, $\vee$ ), and for any $a \in A$ the map $x \mapsto x \wedge a(L \rightarrow L)$ preserves suprema. A map of frames $L \rightarrow L^{\prime}$ is a map of sets $L \rightarrow L^{\prime}$ that preserves finite infima and

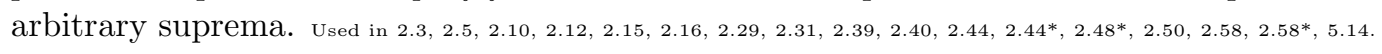

Definition 2.3. The category of locales Loc is defined as Frm

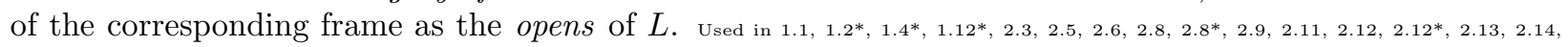

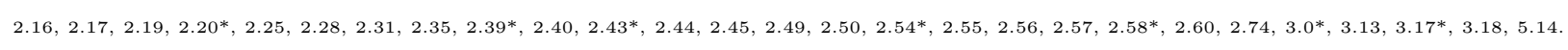

Remark 2.4. To avoid confusion between maps of frames and maps of locales, we use a superscript $*$ for maps of frames, e.g., $f^{*}, g^{*}$, etc.

Remark 2.5. The passage to the opposite category in the definition of Loo is motivated by the desire to make Log like Top. However, most of the actual computations are easier to perform in the category Frm. We automatically transport all notions defined for frames or locales to the opposite category. For instance, below we define open maps of frames, which automatically yields open maps of locales.

Definition 2.6. The functor

$$
\Omega: \text { Top } \rightarrow \text { Log }
$$

from the category of topological spaces and continuous maps to the category of locales sends a topological space $(X, U)$ to its poset of open sets $(U, \subset)$ equipped with the partial order given by inclusion of subsets. It sends a continuous map of topological spaces $f:(X, U) \rightarrow\left(X^{\prime}, U^{\prime}\right)$ to the induced preimage map $f^{*}: U^{\prime} \rightarrow U$.

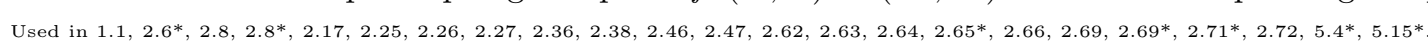

The functor $\Omega$ becomes fully faithful once restricted to the full subcategory of ober topological spaces. 
Definition 2.7. A sober topological space is a topological space for which the operation of closure establishes a bijection from the set of points to the set of irreducible closed subsets, i.e., nonempty closed subsets that cannot be represented as a union of two proper closed subsets. Used in $2.07,2.6 * 2.12 * 2.26,2.37,2.46,2.66$

All Hausdorff topological spaces are sober, and many non-Hausdorff spaces commonly occurring in mathematics, such as the Zariski spectrum of a commutative ring, are also sober.

Definition 2.8. Locales in the image of the functor $\Omega$ are known as spatial. Used in $2.124,2.84,2.19,2.12 ., 2.13$. $2.14,2.26,0.27$.

Thus, to pass from Top to Lod, one must discard the nonsober topological spaces and add the nonspatial locales. Arguably, nonsober spaces carry little practical value for much of mathematics, whereas nonspatial locales are quite important. In fact, below we will define measurable locales, and the only measurable locales that are spatial are precisely the discrete locales, i.e., those obtained by applying $\Omega$ to a discrete topological space. Thus, we are forced to use the pointfree localic formalism in order to formulate our main theorem.

The functor $\Omega$ is a left adjoint functor that fits into the adjunction

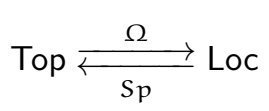

between topological spaces and locales. This adjunction restricts to an adjoint equivalence

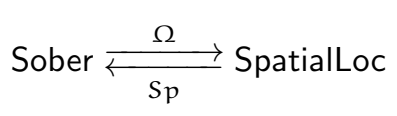

between the categories of sober spaces and spatial locales.

Definition 2.9. The right adjoint functor

$$
\mathrm{Sp}=\mathrm{pt}: \log \rightarrow \text { Top }
$$

sends a locale $L$ to the topological space $S p(L)$ whose set of points $X$ is the set of morphisms of locales $p: 1 \rightarrow L$ and the collection of open sets is constructed as the image of the map of sets $f^{*}: L \rightarrow 2^{X}$, where $f$ is the morphism of locales

$$
\begin{aligned}
& f: \coprod_{p: 1 \rightarrow L} 1 \rightarrow L .
\end{aligned}
$$

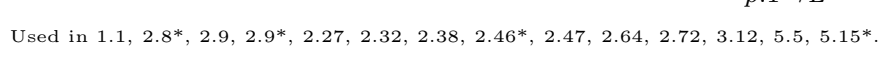

The essential image of $S p$ consists precisely of sober spaces.

Much of general topology can be developed in the setting of locales, see, for instance, Picado-Pultr [2012], which offers a full coverage of basic topics in general topology, including compactness, local compactness, uniformity, paracompactness, completion, metrics, connectedness, real numbers, and localic groups. Furthermore, proofs in the pointfree setting are often more elegant and clear, since we do not have to construct sets of points, which eliminates substantial chunks of arguments. Additional benefits include the elimination of the axiom of choice from most proofs, which allows one to use locales in equivariant or fibered settings codified by arbitrary toposes, where topological spaces simply do not work. We refer the reader to the survey articles by Johnstone [1983] and 1991] for more information.

Remark 2.10. Any map of frames $f^{*}: L^{\prime} \rightarrow L$ preserves suprema, so it admits a right adjoint $f_{*}: L \rightarrow L^{\prime}$. For spatial locales, $f_{*} U$ is the largest open subset of $L^{\prime}$ whose preimage is inside $U$.

Remark 2.11. If $f: L \rightarrow L^{\prime}$ is a map of locales, then we denote the corresponding homomorphism of frames by $f^{*}: L^{\prime} \rightarrow L$ and its right adjoint by $f_{*}: L \rightarrow L^{\prime}$. If $f^{*}$ happens to admit a left adjoint, it is denoted by $f_{!}: L \rightarrow L^{\prime}$. If $L=2^{X}$ and $L^{\prime}=2^{X^{\prime}}$ are discrete locales and the map of locales $L \rightarrow L^{\prime}$ is induced by a map of sets $f: X \rightarrow X^{\prime}$, then the notation $f_{!} \dashv f^{*}$ is consistent with the same notation for images and preimages of subsets with respect to the map of sets $f$.

Definition 2.12. A sublocale of a locale $L$ is a map of locales $f: S \rightarrow L$ such that the map of frames $f^{*}: L \rightarrow S$ is surjective on the underlying sets. Used in $2.12+1,2.50$. 2.74 .

In particular, a subspace $S \subset X$ of a topological space $(X, U)$ equipped with the induced topology yields a sublocale, because open sets in the induced topology are precisely the intersections of $S$ and an element 
of $U$. Conversely, if $S$ is a sublocale of the underlying locale of a sober topological space $X$ and $S$ is a spatia locale, then $S$ is induced by a subspace of $X$. (The underlying locale of a topological space typically also has a lot of sublocales that are not spatial.)

Definition 2.13. The Heyting implication of opens $x, y \in L$ of a locale $L$ is the unique open $x \rightarrow y$ in $L$ such that $w \leq(x \rightarrow y)$ if and only if $(w \wedge x) \leq y$. Used in $2.48 *$

We have $x \rightarrow y=\sup _{w \wedge x \leq y} w$. For spatial locales, $x \rightarrow y$ is the interior of the union of $y$ and the complement of $x$.

Definition 2.14. The pseudocomplement of an open $x \in L$ of a locale $L$ is $\neg x:=(x \rightarrow 0)$.

Thus, $\neg x=\sup _{w \wedge x=0} w$. For spatial locales, $\neg x$ is the interior of the complement of $x$.

Definition 2.15. An open map of frames $f^{*}: L^{\prime} \rightarrow L$ is a map of frames that preserves infima and the Heyting implication (meaning $f^{*}(m \rightarrow n)=\left(f^{*} m \rightarrow f^{*} n\right)$ ), i.e., it is a morphism of complete Heyting

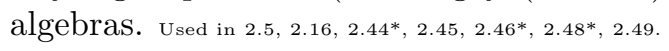

Remark 2.16. If a map of locales $f: L \rightarrow L^{\prime}$ is open, the map of frames $f^{*}: L^{\prime} \rightarrow L$ admits a left adjoint $f_{!}: L \rightarrow L^{\prime}$. One can think of $f_{!}$as sending an open in $L$ to its image in $L^{\prime}$, which is again open because $f$ is an open map. By a theorem of Joyal and Tierney (which is not needed below), one can equivalently define open maps of locales $f: L \rightarrow L^{\prime}$ by requiring that $f^{*}$ admits a left adjoint $f_{!}: L \rightarrow L^{\prime}$ such that the Frobenius reciprocity condition is satisfied: $f_{!}\left(m \wedge f^{*} n\right)=f_{!} m \wedge n$, or, equivalently, $f_{!}\left(a \rightarrow f^{*} b\right)=f_{!} a \rightarrow b$.

Remark 2.17. Any open map $f: X \rightarrow Y$ of topological spaces is sent to an open map $\Omega f: \Omega X \rightarrow \Omega Y$ of locales by the functor Q: Top $\rightarrow$ Log. Conversely, if every point $y \in Y$ has an open neighborhood $U \subset Y$ such that $U \backslash\{y\} \subset Y$ is open (e.g., $Y$ is a $\mathrm{T}_{1}$-space), then whenever $\Omega f$ is an open map of locales, the map $f$ is an open map of topological spaces. Used in 2.46 .

\subsection{Valuations on locales}

Definition 2.19. A positive valuation on a distributive lattice $L$ (e.g., a locale or a Boolean algebra) is a map $\nu: L \rightarrow[0, \infty)$ such that $\nu(0)=0, \nu(x)+\nu(y)=\nu(x \vee y)+\nu(x \wedge y)$, and $x \leq y$ implies $\nu(x) \leq \nu(y)$. A positive valuation is continuous if $\nu$ preserves existing suprema of directed subsets. A complex valuation is a map $\nu: L \rightarrow \mathbf{C}$ such that $\nu(0)=0, \nu(x)+\nu(y)=\nu(x \vee y)+\nu(x \wedge y)$, and we say that it is continuous if $\nu\left(\sup _{x \in I} x\right)=\lim _{x \in I} \nu(x)$ for any directed subset $I \subset L$. A real valuation is a complex valuation that factors through $\mathbf{R} \subset \mathbf{C}$. A valuation is faithful if $\nu(x)=0$ implies $x=0$. Used in $1.1,0.7,2.24,2.12 *, 2.19,2.20,2.20 *$

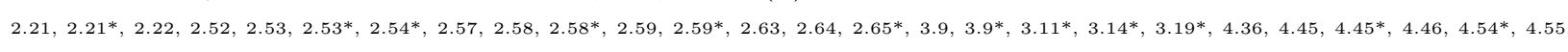
5.4 . 5.11 .

The following definition mimics the usual definition of a measure as a countably additive map from a $\sigma$-algebra, but with countable additivity promoted to complete additivity along the lines of Proposition 4.41.

Definition 2.20. A completely additive valuation $\nu: L \rightarrow[0, \infty)$ on a distributive lattice $L$ is a valuation $\nu: L \rightarrow[0, \infty)$ such that for any family $x: I \rightarrow L$ consisting of pairwise disjoint elements of $L$ (i.e., $x_{i} \wedge x_{j}=0$ whenever $i \neq j$ ) such that $\sup x$ exists, we have

$$
\nu\left(\sup _{i \in I} x_{i}\right)=\sum_{i \in I} \nu\left(x_{i}\right)
$$

where the right sum converges absolutely. Used in $2.20 * 2.21,2.21,2.64$

For arbitrary ocales continuous valuations have better theoretical properties than completely additive valuations. However, for Boolean locales there is no difference.

Lemma 2.21. Assuming the axiom of choice, a map $\nu: A \rightarrow[0, \infty)$ on a Boolean locale (i.e., a complete Boolean algebra) $A$ is a continuous valuation if and only if it is completely additive valuation. Used in 2.64

Proof. Suppose $\nu: A \rightarrow[0, \infty)$ is a continuous valuation. Then for any disjoint family $x: I \rightarrow A$ we have

$$
\nu\left(\sup _{i \in I} x_{i}\right)=\nu\left(\sup _{K \subset I} \sup _{k \in K} x_{k}\right)=\sup _{K \subset I} \nu\left(\sup _{k \in K} x_{k}\right)=\sup _{K \subset I} \sum_{k \in K} \nu\left(x_{k}\right)=\sum_{i \in I} \nu\left(x_{i}\right),
$$


where $K$ runs over all finite subsets of $I$. This shows that $\nu$ is a completely additive valuation.

Conversely, if $\nu: A \rightarrow[0, \infty)$ is a completely additive valuation, i.e.,

$$
\nu\left(\sup _{i \in I} x_{i}\right)=\sum_{i \in I} \nu\left(x_{i}\right)
$$

then substituting $I=\emptyset$ yields $\nu(0)=0$. The identity $z=(z \wedge w) \vee(z \wedge \neg w)$ implies $\nu(x)=\nu(x \wedge y)+\nu(x \wedge \neg y)$ and $\nu(x \vee y)=\nu(y)+\nu(x \wedge \neg y)$, which together imply $\nu(x)+\nu(y)=\nu(x \vee y)+\nu(x \wedge y)$. If $x \geq y$, then $x \wedge y=y$, so $\nu(x)=\nu(x \wedge y)+\nu(x \wedge \neg y)$ implies $\nu(x) \geq \nu(y)$. Thus, $\nu$ is a valuation.

Finally, to show that $\nu$ is continuous, suppose that $S \subset A$ is a directed subset of $A$. We may assume that if $s \in S$ and $s^{\prime} \leq s$, then also $s^{\prime} \in S$. Using Zorn's lemma, choose a maximal disjoint family $P$ that refines $S$. By construction, $\sup P \leq \sup S$. If $\rho=(\sup S) \wedge \neg(\sup P) \neq 0$, then there is $s \in S$ such that $s \wedge \rho \neq 0$ and the family $P \cup\{s \wedge \rho\}$ is a disjoint refinement of $S$, contradicting the maximality of $P$. Thus, $\rho=0$ and $\sup P=\sup S$.

Denote by $Q$ the closure of $P$ under finite joins. Since $S$ is directed, $Q$ refines $S$, so $\sum_{p \in P} \nu(p)=$ $\sup _{q \in Q} \nu(q) \leq \sup _{s \in S} \nu(s)$. On the other hand, for any $s \in S$ we have $s=\sup _{p \in P} s \wedge p$, so $\nu(s)=$ $\sum_{p \in P} \nu(s \wedge p) \leq \sum_{p \in P} \nu(p)$. Thus, $\nu(\sup S)=\nu(\sup P)=\sum_{p \in P} \nu(p)=\sup _{s \in S} \nu(s)$. I

Remark 2.22. In the context of topos theory, where the axiom of choice can be false, only continuous valuations allow for a good theory, with the proviso that $\mathbf{R}$ refers to the lower reals.

\subsection{Stone duality for distributive lattices}

In this section, we briefly review the chain of adjoint equivalences (Proposition 2.27, Proposition 2.31)

$$
\text { Coh } \rightleftarrows \text { CohLod } \rightleftarrows \text { DLat }{ }^{\text {op }}
$$

between coherent spaces, coherent locales, and distributive lattices that serves as a foundation for all Stonetype dualities considered later. Complete proofs of all cited facts are given by Johnstone 1982, §II.3], so we only recall the relevant definitions.

Definition 2.24. A lattice is a poset that admits all finite meets and finite joins. Lattices form a category whose morphisms are maps of posets that preserve finite meets and finite joins. A distributive lattice is a lattice $R$ that satisfies the finite distributive law

$$
x \wedge(y \vee z)=(x \wedge y) \vee(x \wedge z)
$$

for all $x, y, z \in R$. Distributive lattices form a full subcategory DLat of the category of lattices. Used in 2.0. $2.23,2.23,2.29,2.31,2.32,2.34,2.41,2.41$.

Definition 2.25. A compact open of a locale $L$ is an open $a \in L$ such that for any $S \subset L$ with $\bigvee S \geq a$ we have $\bigvee F \geq a$ for some finite $F \subset S$. A locale $L$ is compact if the open $1 \in L$ is a compact open. A topological space $T$ is compact if the locale $\Omega(T)$ is compact, i.e., any open cover of $T$ has a finite subcover. A coherent locale is a locale $L$ such that compact opens of $L$ are closed under finite meets and any open of $L$ is a join of compact opens. Thus, coherent locales are automatically compact. Coherent locales form a category CohLoc whose morphisms are coherent maps of locales, defined as maps of locales $f: L \rightarrow L^{\prime}$ such

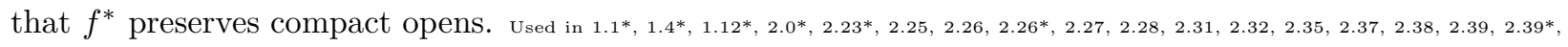
$2.49,2.41,2.41,2.59,4.58,4.61,0.62,5.4,5.11,5.16,5.18$

Definition 2.26. A coherent space is a ober topological space $S$ such that $\Omega(S)$ is a coherent locale. Coherent spaces are automatically compact. Coherent spaces form a category Coh whose morphisms are coherent maps of spaces, defined as continuous maps $f: S \rightarrow S^{\prime}$ such that $\Omega(f)$ is a coherent map of locales. Used in $2.07,2.23 .2 .27,2.37,2.39,2.33,2.40$

The axiom of choice implies that coherent locales are spatial (Johnstone 1982, Theorem II.3.4]), which immediately yields the following result. 
Proposition 2.27. The adjoint equivalence

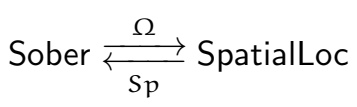

restricts to the adjoint equivalence

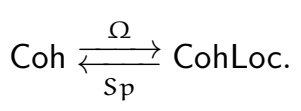

Used in $2.12+2.0 * 2.23 *$

Definition 2.28. The set of compact opens of a locale $L$ is a distributive lattice denoted by COpen $(L)$. Coherent maps of locales preserve compact opens and their finite joins and meets, so we get a functor

$$
\text { COpen: CohLod } \rightarrow \text { DLat } \text { op. }
$$

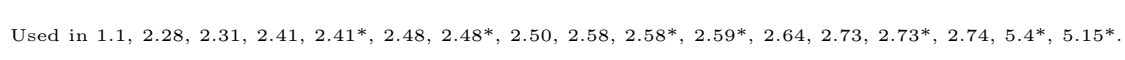

Definition 2.29. An ideal of a distributive lattice $R$ is a subset $I \subset R$ that is closed under finite joins and such that $a \in I$ and $b \leq a$ imply $b \in I$. The set $\operatorname{Idl}(R)$ of all ideals of a distributive lattice $R$ equipped with the inclusion ordering is a frame (Johnstone 1982, Corollary II.2.11]). A morphism $f: R \rightarrow R^{\prime}$ of distributive lattices induces a morphism $\operatorname{Idl}(f): \operatorname{Idl}(R) \rightarrow \operatorname{Idl}\left(R^{\prime}\right)$ that sends an ideal $I \subset R$ to the ideal of $R^{\prime}$ generated by the image of $I$ under $f$. The resulting functor

$$
\text { Idl:DLat } \rightarrow \text { Frm }
$$

is left adjoint to the forgetful functor $\mathrm{Frm} \rightarrow \mathrm{DLat}$ (as shown in the cited result). Used in $2.29,2.30,2.31,5.48$, 5.15

Remark 2.30. The formula $x \rightarrow y=\sup _{w \wedge x \leq y} w$ yields the following formula for the Heyting implication in $\operatorname{Idl}(A)$ :

$$
I \rightarrow J=\{k \in A \mid k I \subset J\} .
$$

In particular,

$$
\neg I=\{k \in A \mid \forall i \in I: k i=0\} .
$$

Used in 2.48 .

Proposition 2.31. (Stone duality for distributive lattices.) There is an adjoint equivalence of categories

$$
\text { CohLod } \underset{\text { Ideal }}{\stackrel{\text { COpen }}{\rightleftarrows} \text { DLat }} \text { ip. }
$$

The functor Ideal is obtained from the functor $I \mathrm{dl}$ op by restricting its codomain to CohLoq. For a coherent locale $L$, the unit $L \rightarrow$ Ideal(COpen $(L))$ is a map of locales whose underlying map of frames sends an ideal $I \subset \operatorname{COpen}(L)$ to the jin of its elements. For a distributive lattice $R$, the counit COpen|(Ideal $(R)) \rightarrow R$ is a morphism in DLat ${ }^{\mathrm{B}}$ such that its opposite morphism in DLat is the map $R \rightarrow$ COpen $\operatorname{lop}(\operatorname{IdV}(R))$ that sends $r \in R$ to the principal ideal of $R$ generated by $r$ (i.e., $\{b \in R \mid b \leq r\}$ ), which is automatically a

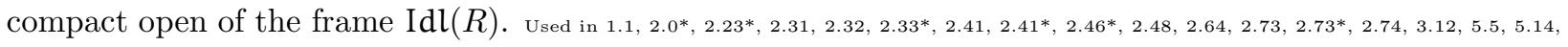
5.15.

Remark 2.32. The composition

$$
\text { DLat } \stackrel{\text { Ideal }}{\longrightarrow \text { CohLog }} \stackrel{\text { Sp }}{\longrightarrow}
$$

is known as the Stone spectrum functor. Used in $1.0 * 2.51 * 0.96 .38$ 


\subsection{Stone duality for Boolean algebras}

In this section we review how the Stone duality for distributive lattices restricts to the following chain of adjoint equivalences (Proposition 2.38, Proposition 2.41):

$$
\text { Stone } \rightleftarrows \text { StoneLod } \rightleftarrows \text { BAlg } 0 \text {, }
$$

essentially due to Stone 1936, 1937.a.

Definition 2.34. A Boolean algebra (alias Boolean ring) is a distributive lattice $R$ in which all elements are complemented: for any $x \in R$ there is $\neg x \in R$ such that $x \wedge \neg x=0$ and $x \vee \neg x=1$. The category of

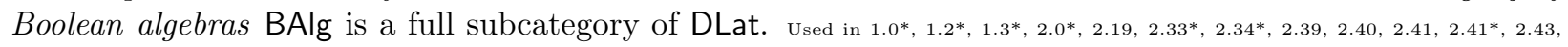

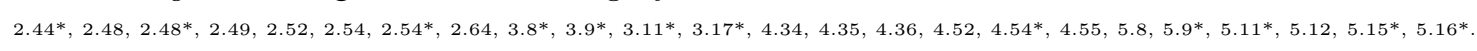

Equivalently, one could define Boolean algebras as rings such that $x^{2}=x$ for all $x$ and the category of Boolean algebras as a full subcategory of the category of rings. In this definition, Boolean algebras are automatically commutative $\mathbf{Z} / 2$-algebras and the ordering can be recovered by defining $x \leq y \equiv(x y=y)$. Recall that by our convention, all rings are unital and all homomorphisms of rings preserve units.

Definition 2.35. A locale $L$ is regular if for any $y \in L$ we have $y=\bigvee_{x \prec x} x$, where $x \prec y$ means $\neg x \vee y=1$. The category StoneLoc of Stone locales is the full subcategory of CohLoc consisting of regular coherent

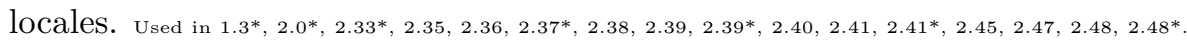

Definition 2.36. A Stone space is a coherent space $T$ such that $\Omega(T)$ is a regular locale (hence a Stone locale). The category of Stone spaces Stone is the full subcategory of Coh consisting of Stone spaces. Used in

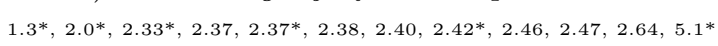

Remark 2.37. Stone originally defined what is now known as Stone spaces using totally disconnected spaces. A topological space is totally disconnected if its only connected subspaces are singletons. Johnstone [1982, Theorem II.4.2] shows that the class of Stone spaces as defined above coincides with the class of compact totally disconnected Hausdorff topological spaces, as used by Stone himself. Alternatively, Stone spaces can be characterized as compact zero-dimensional sober spaces.

The following proposition follows immediately from our definition of Stone spaces and Stone locales.

Proposition 2.38. The adjoint equivalence

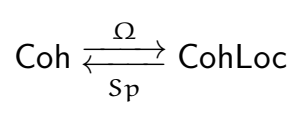

restricts to an adjoint equivalence

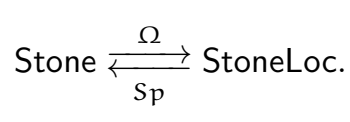

Used in $2.0^{*}, 2.33^{4}$

Lemma 2.39. For a Stone locale $L$, the set of compact opens coincides with the set of clopen (closed and open) elements, i.e., complemented elements of the underlying frame of $L$. Used in $2.397,2.41 ., 2.45,2.48,2.67,2.68$ $2.68,2.69+2.71,2.74,5.47,5.14,5.15 * 5.16 * 5.19 *$

Proof. In a compact locale $L$ any clopen $a \in L$ is a compact open: if for some $S \subset L$ we have $\bigvee S \geq a$, then $S^{\prime}=S \cup\{\neg a\}$ satisfies $\bigvee S=1$, so by compactness of $L$, there is a finite $F^{\prime} \subset S^{\prime}$ such that $\bigvee F^{\prime}=1$, therefore taking $F=F^{\prime} \backslash\{\neg a\} \subset S$ we get $\bigvee F \geq a$.

Conversely, if $a \in L$ is a compact open, then by regularity of $L$ we have $a=\bigvee_{x \prec a} x$, so using compactness of $a$ we can extract a finite subset $F \subset\{x \in L \mid x \prec a\}$ such that $\bigvee F=a$. Since the set $\{z \in L \mid z \prec a\}$ is closed under finite joins, we see that $\bigvee F=a \prec a$, i.e., $\neg a \vee a=1$, so $a$ is clopen. 
Remark 2.40. Maps of frames preserve complemented elements. Thus, maps of locales between Stone locales are automatically coherent, so StoneLod is a full subcategory of Lod, unlike CohLod. Likewise, Stons is a full subcategory of Top, unlike Coh.

Proposition 2.41. (Stone duality for Boolean algebras.) The adjoint equivalence of categories

$$
\text { CohLog } \underset{\text { Ideal }}{\stackrel{\text { COpen }}{\rightleftarrows} \text { DLat }}
$$

restricts to an adjoint equivalence

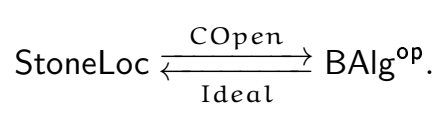

Used in $2.4^{*}, 2.0 * 2.33^{*}, 2.42$.

Proof. It suffices to show that COpen sends Stone locales to Boolean algebras and Ideal sends Boolean algebras to Stone locales. Suppose $L$ is a Stone locale. By Lemma 2.39, compact opens in $L$ are precisely the clopen elements. In particular, every compact open in $L$ is complemented; hence the distributive lattice COpen $(L)$ is a Boolean algebra. Conversely, suppose $A$ is a Boolean algebra. Any principal ideal $(a) \subset A$ is a clopen (hence compact open) element of Ideal $(A)$, with the complement $\neg(a)=(1-a)$. For any open $y \in L$ and a clopen $x \in L$ such that $x \leq y$ we have $x \prec y$ because $\neg x \vee y \geq \neg x \vee x=1$. Thus, for any $I \in \operatorname{Ideal}(A)$ we have $I=\bigvee_{a \in I}(a)$, where $(a) \prec I$; hence the coherent locald Ideal $(A)$ is regulan. I

\subsection{Stonean duality}

Next, we review how the Stone duality for Boolean algebras restricts to a chain of adjoint equivalences (Proposition 2.47, Proposition 2.48)

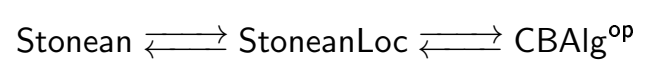

between the (nonfull) subcategories of Stonean spaces, Stonean locales, and complete Boolean algebras, which we refer to as the Stonean duality. Stone's 1937 paper [1937.b] shows that complete Boolean algebras correspond precisely to extremally disconnected Stone spaces under Stone duality.

Definition 2.43. A Boolean algebra is complete if any set of its elements admits a supremum. A morphism of complete Boolean algebras is a complete (i.e., continuous, or supremum-preserving) Boolean homomorphism. The category of complete Boolean algebras CBAlg is a (nonfull) subcategory of the category of Boolean

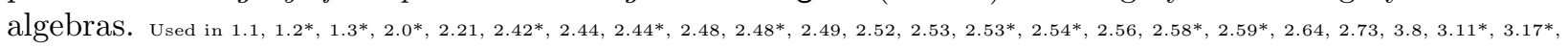
4.33. $4.55 .5 .4^{4}$

A crucial distinguishing feature of Stonean duality is that both categories involved in the adjunction are subcategories of the category of locales. We formulate this as a lemma.

Lemma 2.44. The functor CBAlg $\rightarrow$ Lod defined as the opposite of the forgetful functor CBAlg $\rightarrow$ Frm is a fully faithful functor. Locales $L$ in its essential image BLoc are precisely Boolean locales, i.e., locales for which $a \vee \neg a=1$ for any $a \in L$, or, equivalently, $\neg \neg a=a$ for any $a \in L$. Any map of Boolean locales is

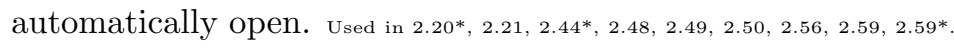

Proof. If $\neg \neg a=a$ for all $a \in L$, then $1=\neg \neg a \vee \neg a=a \vee \neg a$ for all $a \in L$. Conversely, if $a \vee \neg a=1$ for all $a \in L$, then $\neg \neg a=\neg \neg a \wedge(a \vee \neg a)=(\neg \neg a \wedge a) \vee(\neg \neg a \wedge \neg a)=(\neg \neg a \wedge a) \vee 0=\neg \neg a \wedge a=a$ for all $a \in L$. This shows that the two conditions in the statement are equivalent.

First, any $A \in$ CBAlg is indeed a frame: being a complete Boolean algebra, it admits arbitrary suprema and infima, and the map $s \mapsto s \wedge a$ preserves suprema for any $a \in A$ because it admits a right adjoint $t \mapsto(\neg a) \vee t$.

Secondly, any homomorphism $f: A \rightarrow A^{\prime}$ in CBAlg preserves arbitrary suprema and infima, so is indeed a map of frames. Conversely, if $f: A \rightarrow A^{\prime}$ preserves finite infima and arbitrary suprema, then it is a complete homomorphism, so the functor is fully faithful. Furthermore, $f$ preserves the Heyting implication, since in a 
Boolean algebra $x \rightarrow y=\neg x \vee(x \wedge y)=1+x+x y$, and all operations on the right side are preserved by $f$. Thus, $f$ preserves arbitrary infima and the Heyting implication, so $f$ is an open map of frames.

Finally, any complete Boolean algebra is a Boolean locale since $\neg a=1-a$ in this case, so $a \vee \neg a=$ $a \vee(1-a)=1$. Conversely, to show that any Boolean locale has a complete Boolean algebra as its underlying frame, we observe that the condition $\neg \neg a=a$ implies that the underlying Heyting algebra is a Boolean algebra, and hence also a complete Boolean algebra.

Definition 2.45. A locale $L$ is extremally disconnected if $\neg x \vee \neg \neg x=1$ for any $x \in L$, i.e., for any $x \in L$ the element $\neg x \in L$ is clopen. A Stonean locale is an extremally disconnected Stone locale. The category of Stonean locales StoneanLoc has Stonean locales as objects and bpen maps of locales as morphisms. It is a

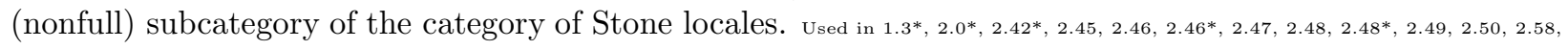
$2.59,2.59 \cdot 2.612 .72,2.73$

If we apply this definition to a locale that comes from a topological space, the condition $\neg x \vee \neg \neg x=1$ boils down to saying that the closure $\bar{x}$ is its own interior, i.e., the closure of any open $x$ is open.

Definition 2.46. A topological space $T$ is extremally disconnected if $\Omega(T)$ is an extremally disconnected locale. A Stonean space is an extremally disconnected Stone space, equivalently, a sober topological space $T$ such that $\Omega(T)$ is a Stonean locale. The category of Stonean spaces Stonean has Stonean spaces as objects and open continuous maps as morphisms. It is a (nonfull) subcategory of the category of Stone spaces. Used

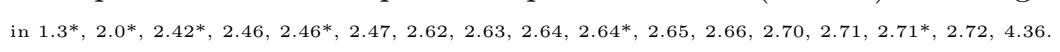

A result of Edwards [2002.b, Theorem 5.1] shows that (in our notation) morphisms in the image of the composition $5 \mathrm{p} \circ \mathrm{Ideal} \circ$ ProjLod are precisely the open maps, which motivates the choice of open maps as morphisms between Stonean locales.

Our definition of Stonean locales and Stonean spaces together with Remark 2.17 immediately implies the following.

Proposition 2.47. The adjoint equivalence

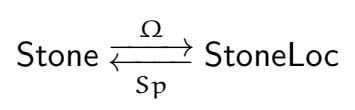

restricts to an adjoint equivalence

Used in $2.0 *$ 2.42.

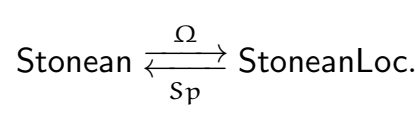

Proposition 2.48. (Stonean duality.) The adjoint equivalence

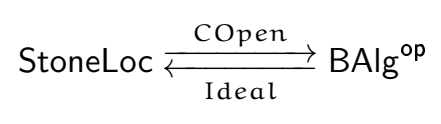

restricts to an adjoint equivalence

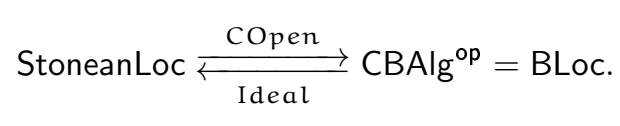

Used in $2.0^{*}, 2.42^{*}, 2.48^{*} 2.51$.

Proof. It suffices to show that both functors preserve the given subcategories. Johnstone 1982, Lemma III.3.5] shows that both functors preserve objects of the given subcategories. We include a short proof of this below together with a proof of preservation of morphisms.

We work with the opposite adjunction

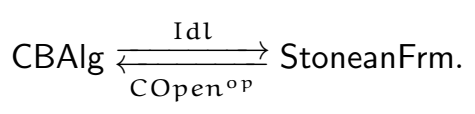


An element $x \in S$ of an extremally disconnected frame $S$ is clopen if and only if $x=\neg \neg x$. Furthermore, $\neg \neg x$ is the smallest clopen element greater than or equal to $x$. If $S \in$ StoneanFrm, then the Boolean algebra COpen $1(S)$ is complete because $\sup R=\neg \neg \bigvee R$ gxists for any $R \subset$ COpen op $(S)$. The supremum on the left is computed in the Boolean algebra COpen $(S)$, whereas the join on the right is computed in the poset $S$.

Recall that a map of frames $f: S \rightarrow S^{\prime}$ is open if it preserves infima and Heyting implications, and hence also negations. If $f: S \rightarrow S^{\prime}$ is an open map of Stonean frames, the induced map of Boolean algebras

$$
\text { COpen op }(f): \text { COpen op }(S) \rightarrow \text { COpen op }\left(S^{\prime}\right)
$$

preserves suprema: given $R \subset$ COpen op $(S)$, we have $\sup R=\neg \neg \bigvee R$, so

$$
f(\sup R)=f(\neg \neg \bigvee R)=\neg \neg f(\bigvee R)=\neg \neg \bigvee f(R)=\sup f(R) .
$$

This shows that the functor COpen restricts to the claimed subcategories.

For any $A \in$ CBAlg, the Stone frame $\operatorname{Idl}(A)$ is extremally disconnected because $\neg \neg I \vee \neg I=A$ for any ideal $I \subset A$. Indeed, according to Remark 2.30, $\neg I=\{k \in A \mid \forall i \in I: k \wedge i=0\}$. Since $A$ is complete, we can rewrite this expression as $\neg I=\{k \in A \mid k \wedge \sup I=0\}$, i.e., $\neg I$ is the principal ideal of $A$ generated by the element $1-\sup I$. Now $\neg \neg I$ is the principal ideal of $A$ generated by the element sup $I$. Therefore, $\neg I \vee \neg \neg I=A$.

Finally, the functor Idl sends a complete homomorphism $g: A \rightarrow A^{\prime}$ of complete Boolean algebras to an open map of frames $\llbracket \mathrm{dd}(g): \operatorname{Id}(A) \rightarrow \llbracket \mathrm{d} d\left(A^{\prime}\right)$. First, $\amalg \mathrm{d} d(g)$ preserves infima; the nontrivial part is

$$
\inf _{k} \operatorname{Idl}(g)\left(I_{k}\right) \leq \operatorname{Idl}(g)\left(\inf _{k} I_{k}\right) .
$$

Take an element $x^{\prime}$ from the left side and recall that infima of ideals coincide with intersections, i.e., for any $k$ we have $x^{\prime} \in \operatorname{Idl}(g)\left(I_{k}\right)$, which means that there is $y_{k} \in I_{k}$ such that $x^{\prime} \leq g\left(y_{k}\right)$. Now $x^{\prime} \leq \inf _{k} g\left(y_{k}\right)=$ $g\left(\inf _{k} y_{k}\right)$ by completeness of $g$, so $x^{\prime} \in \llbracket \mathrm{Id}(g)\left(\inf _{k} I_{k}\right)$. Secondly, Id $(g)$ preserves Heyting implications; the nontrivial part is

$$
\operatorname{Idl}(g)(I) \rightarrow \operatorname{Idl}(g)(J)) \leq \operatorname{Idl}(g)(I \rightarrow J) .
$$

Take an element $x^{\prime}$ from the left side; by Remark 2.30 we have $x^{\prime} \wedge \llbracket \mathrm{dl}(g)(I) \leq \amalg \mathrm{dl}(g)(J)$, so for any $i \in I$ there is $j \in J$ such that

$$
x^{\prime} \wedge g(i) \leq g(j) \Longleftrightarrow x^{\prime} \leq(g(i) \rightarrow g(j))=g(i \rightarrow j) \Longleftrightarrow g_{!}\left(x^{\prime}\right) \leq i \rightarrow j \Longleftrightarrow g_{!}\left(x^{\prime}\right) \wedge i \leq j .
$$

Thus, $g_{!}\left(x^{\prime}\right) \wedge I \leq J$, so $g_{!}\left(x^{\prime}\right) \in I \rightarrow J$. Now $x^{\prime} \leq g\left(g_{!}\left(x^{\prime}\right)\right)$ by adjunction $g_{!} \dashv g$, so $x^{\prime} \in \operatorname{Id} l(g)(I \rightarrow J)$. I

Remark 2.49. According to Lemma 2.44, both sides of the restricted adjunction StoneanLod $\rightleftarrows$ CBAlg subcategories of Lod, with the subcategory CBAlg $\mathrm{OP}=\mathrm{BLod}$ being a full subcategory and the subcategory StoneanLe being a nonfull subcategory (its morphisms are open maps of locales). As shown there, morphisms of CBAlg 9 are automatically open, so both sides are full subcategories of the category of locales and open maps. The two sides are drastically different in terms of their relation to topological spaces: whereas in presence of the rxiom of choice all Stonean locales are spatial, i.e., correspond to topological spaces, the locales in CBAlg $\mathrm{op}=\mathrm{BLog}$ are never spatial unless they are complete atomic Boolean algebras, i.e., Boolean algebras of subsets of a given set $X$. In fact, any complete Boolean algebra splits as a product of a complete atomic Boolean algebra and a complete atomless Boolean algebra. The latter has no points at all when interpreted as a locale. Another difference is that Stonean locales are always compact, whereas the only compact locales in CBAlge are Boolean algebras of subsets of a finite set.

Remark 2.50 Compact opens of any Stonean frame $S$ are characterized by the property $s=\neg \neg s$, so we have COpen $(S)=\neg \neg S$, where for any locale $L$ we define

$$
\neg \neg L=\{s \in L \mid s=\neg \neg s\}=\{\neg \neg s \mid s \in L\} .
$$

The double negation map $\neg \neg: L \rightarrow \neg \neg L$ is a surjective homomorphism of frames and we have a canonical embedding of locales $\neg \neg L \rightarrow L$. The sublocale $\neg \neg L$ of $L$ is also known as the double negation sublocale of $L$ and is the largest sublocale of $L$ that is a Boolean locale. It can also be characterized as the intersection of all dense sublocales of $L$, where a dense sublocale is a sublocale that is not contained in any closed sublocale other than $L$. Used in 2.7 . 


\subsection{Hyperstonean duality}

Finally, we show how the Stonean duality restricts to a chain of adjoint equivalences from Theorem 1.1 (Proposition 2.72, Proposition 2.73)

$$
\text { HStonean } \rightleftarrows \text { HStoneanLod } \rightleftarrows \text { LBAlg }
$$

between the full subcategories of hyperstonean spaces, hyperstonean locales, and localizable Boolean algebras, which we refer to as the hyperstonean duality. Hyperstonean spaces were introduced and studied by Dixmier [1951.a], who in particular shows [1951.a, Théorème 2] that hyperstonean spaces are precisely the Gelfand spectra of commutative von Neumann algebras, equivalently, Stone spectra of localizable Boolean algebras.

Definition 2.52. A localizable Boolean algebra is a Boolean algebra $A$ that is complete and in which the element $1 \in A$ equals the supremum of all $a \in A$ such that the complete Boolean algebra $a A$ admits a (finite) faithful continuous valuation $a A \rightarrow[0, \infty)$. The category of localizable Boolean algebras LBAlg is a

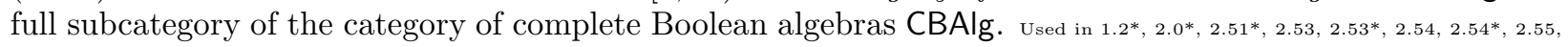

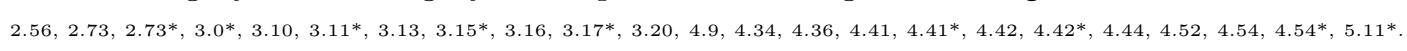

Lemma 2.53. A complete Boolean algebra $A$ is localizable if and only if for any nonzero $b \in A$ there is a continuous valuation $\mu: A \rightarrow[0, \infty)$ such that $\mu(b) \neq 0$. Used in 2.54 . 3.11 .

Proof. Suppose $A$ is localizable, i.e., the supremum of all $a \in A$ such that the complete Boolean algebra $a A$ admits a (finite) faithful continuous valuation equals $1 \in A$. For any nonzero $b \in A$ we have $b=1 \cdot b=$ $\left(\sup _{a} a\right) b=\sup _{a}(a b)$, so one of $a b$ must be nonzero. Fixing such an $a$, there is a faithful continuous valuation $\mu: a A \rightarrow[0, \infty)$. It extends to a continuous valuation $\nu: A \rightarrow[0, \infty)$ via the formula $\nu(c)=\mu(a c)$. We have $\nu(b)=\mu(a b) \neq 0$ since $\mu$ is faithful on $a A$.

Conversely, if for any nonzero $b \in A$ there is a continuous valuation $\mu: A \rightarrow[0, \infty)$ such that $\mu(b) \neq 0$, then denote by $c$ the supremum of all $a \in A$ such that the complete Boolean algebra $a A$ admits a faithfu] continuous valuation. If $c \neq 1$, then there is a continuous valuation $\mu: A \rightarrow[0, \infty)$ such that $\mu(1-c) \neq 0$. By continuity of $\mu$ there is a maximum $d \in A$ such that $\mu(d)=0$, so $\mu$ is faithful on $(1-d) A$ and vanishes on $d A$. By definition of $c$, we must have $1-d \leq c$, but then

$$
\mu(1-c)=\mu((1-c)(1-d+d))=\mu((1-c)(1-d))+\mu((1-c) d)=\mu((1-c)(1-d)) \leq \mu((1-c) c)=0,
$$

which contradicts $\mu(1-c) \neq 0$. I

Proposition 2.54. The category LBAlg admits all small products and the forgetful functor LBAlg $\rightarrow$ BAlg preserves small products.

Proof. Complete Boolean algebras are closed under small products in the category BAlg because suprema in a product $\prod_{i \in I} A_{i}$ of complete Boolean algebras $A_{i}$ can be computed indexwise with respect to $I$. Localizable Boolean algebras are closed under small products in the category BAlg by Lemma 2.53: if $b \in \prod_{i \in I} A_{i}$ is nonzero, then for at least one $j \in I$ the projection $b_{j} \in A_{j}$ is nonzero. Since $A_{j}$ is localizable, we can find a continuous valuation $\mu_{j}: A_{j} \rightarrow[0, \infty)$ such that $\mu_{j}\left(b_{j}\right) \neq 0$. Composing $\mu_{j}$ with the projection map $\prod_{i \in I} A_{i} \rightarrow A_{j}$, we get a continuous valuation $\mu: \prod_{i \in I} A_{i} \rightarrow[0, \infty)$ such that $\mu(b) \neq 0$. Thus, $\prod_{i \in I} A_{i}$ is localizable. I

We now define the category of measurable locales, which is in the same relation to traditional measurable spaces as locales are to traditional topological spaces. Just like for locales we express everything in terms of the complete lattice of open sets, for measurable locales we express everything in terms of the complete lattice of equivalence classes of measurable sets modulo negligible sets.

Definition 2.55. The category MLoc of measurable locales is the full subcategory of the category Lod of

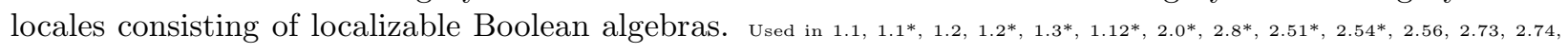

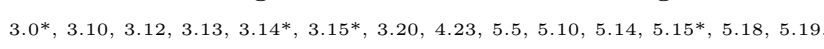

Remark 2.56. According to temma 2.44 the inclusion CBAlgop $\rightarrow$ Log is a fully faithful functor, and hence

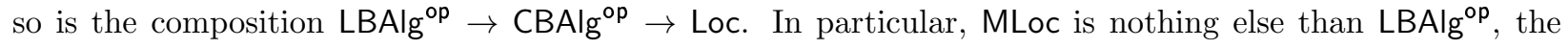


opposite category of localizable Boolean algebras. Also, MLod is a full subcategory of the category BLog of Boolean locales since LBAlg is a full subcategory of CBAlg.

Definition 2.57. A normal valuation on a locale $L$ is a continuous valuation $\nu$ such that $\nu(\neg \neg a)=\nu(a)$

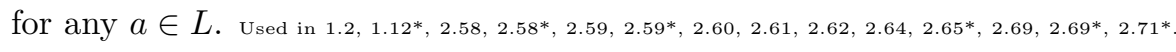

Lemma 2.58. For any Stonean locale $L$, the restriction along the map of posets $\operatorname{COpen}(L) \rightarrow L$ establishes a bijection from the set of hormal valuations on $L$ to the set of continuous valuations on COpen $(L)$. The inverse map is given by precomposing with the map of frames $\neg \neg: L \rightarrow$ COpen $(L)$. Used in $1.24,2.59$, . 2.64 2.73. 5.6.

Proof. The inclusion COpen $(L) \rightarrow L$ preserves finite meets and finite joins, so any valuation $\mu$ on $L$ restricts to a valuation $\nu$ on COpen $(L)$. Given a directed subset $S \subset \operatorname{COpen}(L)$, we have $\sup S=\neg \neg \bigvee S$, where the supremum on the left is taken in the complete Boolean algebra COpen $(L)$, whereas the join on the right is taken in the locale $L$. Given a normal valuation $\mu$ on $L$, we have $\mu(\neg \neg \bigvee S)=\mu(\bigvee S)=\lim _{s \in S} \nu(s)$, which proves that the restriction $\nu$ of $\mu$ to $\operatorname{COpen}(L)$ is a continuous valuation.

Conversely, given a continuous valuation $\nu$ on COpen $(L)$, precomposing it with the map of frames $\neg \neg: L \rightarrow \operatorname{COpen}(L)$ yields a continuous valuation on $L$ that is normal by construction.

The map of frames $\neg \neg: L \rightarrow$ COpen $(L)$ is identity on COpen $(L)$, which immediately implies that the two constructed maps are inverse to each other, so we indeed have a bijection between normal valuations on $L$ and continuous valuations on COpen $(L)$.

Lemma 2.59. (Hahn-Jordan decomposition of normal valuations.) Given a normal valuation $\nu: L \rightarrow \mathbf{R}$ on a Stonean locale $L$, define $\nu_{+}$respectively $\nu_{-}$as the supremum of all $a \in L$ such that $\nu$ respectively $-\nu$ restricted to $\{b \in L \mid b \leq a\}$ is a positive normal valuation. Then the above condition on $a \in L$ is equivalent to $a \leq \nu_{+}$respectively $a \leq \nu_{-}$, in particular, both suprema are also maxima. Furthermore, $\nu_{+} \vee \nu_{-}=1$ (Hahn decomposition of $\nu$ ) and $\nu_{+} \wedge \nu_{-}$is the maximal element $a \in L$ such that $\nu$ vanishes on $\{b \in L \mid b \leq a\}$. Define $\nu^{+}, \nu^{-}: L \rightarrow[0, \infty)$ by setting $\nu^{+}(x)=\nu\left(\nu_{+} \wedge x\right)$ and $\nu^{-}(x)=-\nu\left(\nu_{-} \wedge x\right)$. Then $\nu^{+}$and $\nu^{-}$are positive normal valuations on $L$ and $\nu=\nu^{+}-\nu^{-}$(Jordan decomposition of $\nu$ ). We also set $|\nu|=\nu^{+}+\nu^{-}$ and $\|\nu\|=|\nu|(1)$, the latter known as the variation norm of $\nu$. The above statements also hold if $\nu: L \rightarrow \mathbf{R}$ is a continuous valuation on a Boolean locale $L$. Continuous valuations on Boolean locales are automatically normal valuations.) For complex valuations, we get a canonical decomposition into four summands. Used in 3.9. (3.14.

Proof. Lemma 2.58 establishes a bijection between normal valuations on a Stonean locale $L$ and continuous valuations on the complete Boolean algebra COpen $(L)$. Since $\operatorname{COpen}(L) \subset L$ and $\nu_{+}, \nu_{-} \in \overline{C O p e n}(L)$ because $\nu$ is a normal valuation, it suffices to treat the case of continuous valuations on Boolean locales.

Proceeding exactly as in the proof of the classical Hahn-Jordan decomposition (see, for example, Fremlin [2001, Theorem 231E], whose proof we follow here), we first show that $\nu$ must be bounded. Below, $a \backslash b$ means $a \wedge(1-b)$. Set $N(x)=\sup \{|\nu(y)| \mid y \leq x\}$. If $N(1)=\infty$, construct a sequence $\left\{E_{n}\right\}_{n \geq 0}$ such that $N\left(E_{n}\right)=\infty$ as follows. Set $E_{0}=1 \in L$. Having constructed $E_{n} \in L$ with $N\left(E_{n}\right)=\infty$, choose $F_{n} \leq E_{n}$ such that $\left|\nu\left(F_{n}\right)\right| \geq\left|\nu\left(E_{n}\right)\right|+1$, then also $\left|\nu\left(E_{n} \backslash F_{n}\right)\right| \geq 1$. Since $N\left(E_{n}\right)=\infty$, we must have $N\left(F_{n}\right)=\infty$ or $N\left(E_{n} \backslash F_{n}\right)=\infty$. In the former case, set $E_{n+1}=F_{n}$ and in the latter, set $E_{n+1}=E_{n} \backslash F_{n}$. We have $N\left(E_{n+1}\right)=\infty$ and $\left|\nu\left(E_{n} \backslash E_{n+1}\right)\right| \geq 1$. The sequence $\left\{E_{n} \backslash E_{n+1}\right\}_{n \geq 0}$ is disjoint, so $\nu\left(\bigvee_{n \geq 0} E_{n} \backslash E_{n+1}\right)=$ $\sum_{n \geq 0} \nu\left(E_{n} \backslash E_{n+1}\right)$ is finite, which implies $\nu\left(E_{n} \backslash E_{n+1}\right) \rightarrow 0$ as $n \rightarrow \infty$, and yet $\left|\nu\left(E_{n} \backslash E_{n+1}\right)\right| \geq 1$ for all $n \geq 0$, a contradiction. Thus, $N(1)<\infty$ and $\nu$ is bounded.

Next, we show that $\nu$ attains its supremum $\gamma$. Choose $E_{n} \in L$ such that $\nu\left(E_{n}\right) \geq \gamma-2^{-n}$, for all $n \geq 0$. Set $F_{m, n}=\bigwedge_{m \leq i \leq n} E_{i}$. We have $\nu\left(F_{m, n}\right) \geq \gamma-2^{1-m}+2^{-n}$ (shown by induction on $n$ ). Set $G_{m}=\bigwedge_{n \geq m} F_{m, n}$. We have $\nu\left(G_{m}\right) \geq \gamma-2^{1-m}$ because $F_{m, n} \geq F_{m, n+1}$ for all $m \geq 0$. Set $H=\bigvee_{m>0} G_{m}$. Since $G_{m} \leq G_{m+1}$, we have $\nu(H) \geq \gamma$, so $\nu(H)=\gamma$. For any $K \leq H$ we have $\nu(H)-\nu(K)=\nu(H \backslash K) \leq$ $\gamma=\nu(H)$, so $\nu(K) \geq 0$. For any $K \leq 1-H$ we have $\nu(H)+\nu(K)=\nu(H \vee K) \leq \gamma=\nu(H)$, so $\nu(K) \leq 0$.

Consider the element $Q=1-\left(\nu_{+} \vee \nu_{-}\right)$. The above construction yields some $H \leq Q$ on which $\nu$ attains its supremum on $\{H \mid H \leq Q\}$ and for any $K \leq H$ we have $\nu(K) \geq 0$. Thus, $H=0$ since otherwise $\nu_{+}$ could be replaced by $\nu_{+}+H$, yielding a contradiction with the definition of $\nu_{+}$. Hence, $\nu(K) \leq 0$ for all $K \leq Q$. Likewise, $\nu(K) \geq 0$, and hence $\nu(K)=0$ for all $K \leq Q$. Thus, $Q=0$ since otherwise $\nu_{+}$could be replaced by $\nu_{+}+Q$, yielding a contradiction with the definition of $\nu_{+}$. Hence, $\nu_{+} \vee \nu_{-}=1$. 
Both $\nu^{+}$and $\nu^{-}$are continuous valuations on $L$ because $x \mapsto \nu_{+} \wedge x$ and $x \mapsto \nu_{-} \wedge x$ are morphisms of lattices that preserve suprema of directed subsets. Also, $\nu^{+}$and $\nu^{-}$are positive because $\nu^{+}(x)=\nu\left(\nu_{+} \wedge x\right)=$ $\sup _{a} \nu(a \wedge x) \geq 0$, where $a$ runs over all elements of $L$ such that $\nu$ restricted to $\{b \in L \mid b \leq a\}$ is a positive continuous valuation.

We have

$\nu(x)=\nu\left(\left(\nu_{+} \vee \nu_{-}\right) \wedge x\right)=\nu\left(\left(\nu_{+} \wedge x\right) \vee\left(\nu_{-} \wedge x\right)\right)=\nu\left(\nu_{+} \wedge x\right)+\nu\left(\nu_{-} \wedge x\right)-\nu_{(}\left(\nu_{+} \wedge \nu_{-} \wedge x\right)=\nu^{+}(x)-\nu^{-}(x)$,

which establishes the Jordan decomposition of $\nu$. I

Corollary 2.60. Complex normal valuations on a locale $L$ are precisely elements in the linear span of positive normal valuations on $L$.

Definition 2.61. The category HStoneanLoc of hyperstonean locales is the full subcategory of StoneanLog consisting of Stonean locales $L$ that admit sufficiently many normal valuations, meaning that for any $a \in$

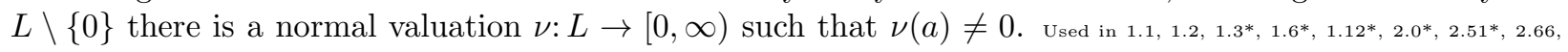
$2.69,2.71+2.72,2.73,2.73+2.74,3.12,5.5$.

Definition 2.62. A finite normal measure $\mu$ on a Stonean space $S$ is a Borel measure $\mu$ on $S$ (i.e., a measure on the $\sigma$-algebra generated by open subsets of $S$ ) whose restriction to open subsets of $S$ is a normal valuation

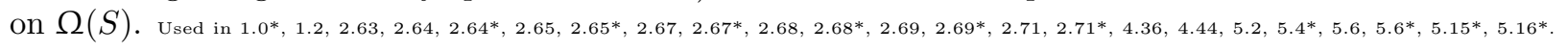

Recall that a subset $A$ of a topological space $X$ is rare (alias nowhere dense) if the interior of the closure of $A$ is empty and meager (alias first category) if it is a countable union of rare sets.

Remark 2.63. The restriction of a finite Borel measure on $S$ to $Q(S)$ is automatically a valuation. The condition that this valuation is continuous is also known as $\tau$-additivity, $\tau$-regularity, or $\tau$-smoothness in measure theory. On a compact regular topological space, finite $\tau$-smooth Borel measures coincide with finite Radon measures, so we define normal measures (not necessarily finite) on a Stonean space $S$ as Radon measures that vanish on all rare subsets of $S$. With such a reformulation it is clear that a hormal measure on $S$ yields an ordinary measure on the enhanced measurable space $\mathrm{TM}(S)$ constructed in Definition 5.2.

Remark 2.64. For a Boolean algebra $A$, finitely additive maps $A \rightarrow[0, \infty)$ are in a canonical bijective correspondence with (positive finite) Radon measures on the Stone space $\operatorname{Sp}]$ Ideal $(A)$ ) by Fremlin [2003, Proposition 416Q]. If $A$ is a complete Boolean algebra, then by Lemma 2.21, completely additive valuations $A \rightarrow[0, \infty)$ are precisely continuous valuations $A \rightarrow[0, \infty)$, by Lemma 2.58, continuous valuations on $A$ are in a canonical bijective correspondence with normal valuations on Ideal $(A)$, and by Remark 2.70, hormal valuations on Ideal $(A)$ are in a canonical bijective correspondence with hormal measures on $\operatorname{Sp} \mid$ Ideal $(A))$. Thus, the condition that a (positive finite) Radon measure on a Stonean space $S$ vanishes on all rare subsets, i.e., is normal, is precisely equivalent to the condition that the corresponding valuation on COpen $\Omega(S)$ ) is completely additive, equivalently, continuous.

We now show that our definition of normal measures on Stonean spaces is equivalent to Dixmier's definition 1951.a, Definition 1]. This lemma is not used anywhere else.

Lemma 2.65. The set of normal measures on a Stonean space $S$ coincides with the set of positive Radon measures $\mu$ such that the integration map $C(S, \mathbf{R}) \rightarrow \mathbf{R}$ with respect to $\mu$ preserves suprema of bounded directed subsets.

Proof. According to Dixmier 1951.a, Proposition 1], a measure $\mu$ is a positive Radon measure for which the integration map $C(S, \mathbf{R}) \rightarrow \mathbf{R}$ with respect to $\mu$ preserves suprema of bounded directed subsets if and only if $\mu$ is a positive Radon measure that vanishes on all are (alias nowhere dense) subsets of $S$. Suppose $\mu$ satisfies these equivalent conditions. Denote by $\nu$ the restriction of $\mu$ to $\Omega(S)$, which is a valuation because $\mu$ is finitely additive. Radon measures are $\tau$-smooth, which means precisely that $\nu$ is a continuous valuation. Given an open subset $U \subset S$, the set $\bar{U} \backslash U$ is rare, so $\mu(\bar{U})=\mu(U)$, which shows that $\nu(\neg \neg a)=\nu(a)$ for any $a \in L$ if we recall that $\bar{U}=\neg \neg U$ for any $U \in \Omega(S)$. Thus, $\nu$ is a normal valuation on $\Omega(S)$.

Conversely, consider a (finite positive) Borel measure $\mu$ on $S$ whose restriction to the open subsets of $S$ is a normal valuation on $\Omega(S)$. First, $\mu$ vanishes on all rare subsets $R \subset S$ because $\mu(S)=\mu(S \backslash \bar{R})$ since 
$\neg \neg(S \backslash \bar{R})=S$, so $\mu(\bar{R})=0$ and $\mu(R)=0$. Secondly, the Borel measure $\mu$ is $\tau$-smooth by definition of a continuous valuation. Every $\tau$-smooth Borel measure on a compact topological space is a Radon measure, which completes the proof. I

Definition 2.66. A hyperstonean space is a sober space $S$ such that $\Omega(S)$ is a hyperstonean locall. The category of hyperstonean spaces $\mathrm{HStonean}$ is a full subcategory of the category of Stonean spaces. Used in 1.0 .

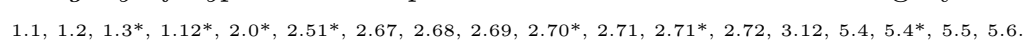

Lemma 2.67. In a hyperstonean space $S$, the class of rare subsets, the class of meager subsets, and the class of measurable subsets (i.e., symmetric differences of open and meagen subsets) on which every normal measure vanishes all coincide. Used in 2.68 .

Proof. (See Dixmier 1951.a, Proposition 5].) All tare subsets are meagen and normal measures vanish on all meager subsets by definition. Suppose $A \subset S$ is a measurable subset on which all normal measures vanish. We have to show that $A$ is rare. The set $\bar{A} \backslash A$ is rare, so all normal measures vanish on $\bar{A}$, and hence also on the clopen subset $\bar{A}^{\circ}$. This means that $\bar{A}^{\circ}=\emptyset$, i.e., $\bar{A}$ is rare, so $A$ is also rare. I

Lemma 2.68. Given a hyperstonean space $S$, the following classes of sets (henceforth known as measurable sets) coincide:

- symmetric differences of clopen sets and rare set;;

- symmetric differences of closed sets and rare sets;

- symmetric differences of open sets and rare sets;

- unions of open sets and rare sets (can be assumed to be disjoint);

- differences of closed sets and rare sets (the former can be assumed to contain the latter).

The class $M$ of measurable sets is a $\sigma$-algebra of subsets of $S$ (Definition 4.2). Every element $X$ of $M$ admits a unique presentation as the symmetric difference of a clopen set and a rare set. Furthermore, for any measurable subset $X$ of $S$, the symmetric difference of any two of the five sets $X, X, X^{\circ}, \overline{X^{\circ}},(\bar{X})^{\circ}$ is a rare set. The class $N$ of rare sets is a $\sigma$-ideal (Definition 4.3) of $M$. Used in 2.69* 5.3.7. 5.15.

Proof. The interior of a closed subset $F$ is a clopen subset $F^{\prime}$ and $F \backslash F^{\prime}$ is a rare set. The closure of an open subset $U$ is a clopen subset $U^{\prime}$ and $U^{\prime} \backslash U$ is a rare set. Thus, $U=U^{\prime} \oplus\left(U^{\prime} \backslash U\right)$ and $F=F^{\prime} \oplus\left(F \backslash F^{\prime}\right)$ are symmetric differences of a clopen set and a rare set, so the first three classes coincide.

We have $U \cup R=U \oplus(R \backslash U)$ and $F \backslash R=F \oplus(R \cap F)$, so the last two classes consist of measurable sets. Conversely, the relations $U \oplus R=(U \backslash \bar{R}) \cup((R \backslash U) \cup((\bar{R} \backslash R) \cap U))$ (the union is disjoint) and $F \oplus R=(F \cup \bar{R}) \backslash((R \cap F) \cup(\bar{R} \backslash(R \cup F)))$ (in the outer difference, the former set contains the latter) show that any measurable set belongs to the fourth respectively fifth class. Thus, all five classes coincide.

By construction, rare sets are measurable and by Lemma 2.67, the class of rare sets coincides with the class of meager sets, which is closed under passage to subsets and countable unions, and hence is a $\sigma$-ideal. Since $S \backslash(U \oplus R)=(S \backslash U) \oplus R$ for any open set $U$ and rare set $R$, measurable sets are closed under complements. Since $\bigcup_{i \geq 0}\left(U_{i} \cup R_{i}\right)=\bigcup_{i \geq 0} U_{i} \cup \bigcup_{i \geq 0} R_{i}$, the set $\bigcup_{i \geq 0} U_{i}$ is open, and the set $\bigcup_{i \geq 0} R_{i}$ is meagen, and hence also rare, measurable sets are closed under countable unions. Hence, the class of measurable sets is a $\sigma$-algebra.

The presentation of a measurable set as the symmetric difference of a clopen subset $U$ and a $\operatorname{are}$ subset $R$ is unique: if $U \oplus R=U^{\prime} \oplus R^{\prime}$, then $U \oplus U^{\prime}=R \oplus R^{\prime}$ is clopen and rare. Clopen rare sets are empty, so $U=U^{\prime}$ and $R=R^{\prime}$.

The relations $\overline{F \backslash R} \subset F$ and $(U \cup R)^{\circ} \supset U$, combined with the fact that subsets of rare sets are again rar, show that the sets $X \oplus \bar{X}$ and $X \oplus X^{\circ}$ are rare, which implies the claim about 5 sets obtained from $X$ in the main statement. I

Lemma 2.69. Given a hyperstonean space $S$, there is a canonical bijective correspondence between hormat measures on $S$ and normal valuations on the hyperstonean locale $\Omega(S)$, given by restricting normal measures on $S$ to open subsets of $S$. Used in $1.2^{+} \mid \frac{2.79}{\text {. }}$.

Proof. By definition, a normal measure on $S$ restricts to a normal valuation on $\Omega(S)$. To show that a normal valuation on $\Omega(S)$ extends to a unique normal measure on $S$, observe that by Lemma 2.68 , any measurable subset $A$ of $S$ has a unique presentation as the symmetric difference of a clopen subset $U$ and a rare subset $R$. For any normal measure $\mu$ that extends $\nu$ we must have $\mu(A)=\mu(U \oplus R)=\mu(U)=\nu(U)$, 
which shows uniqueness. To show existence, we set $\mu(A)=\nu(U)$ and claim that $\mu$ is a normal measure. By construction, $\mu$ extends $\nu$ and vanishes on all meager subsets. To show additivity for a countable family of disjoint measurable subsets $A_{i} \subset S(i \geq 0)$, present $A_{i}$ as the symmetric difference of a clopen subset $U_{i} \subset S$ and a rare subset $R_{i} \subset S$. For any $i \neq j$, the intersection $U_{i} \cap U_{j}$ is clopen and rare, hence empty. Thus, both $\left\{U_{i}\right\}_{i \geq 0}$ and $\left\{A_{i}\right\}_{i \geq 0}$ are disjoint families. We have

$$
\bigcup_{i \geq 0} A_{i} \subset \bigcup_{i \geq 0}\left(U_{i} \cup R_{i}\right)=\bigcup_{i \geq 0} U_{i} \cup \bigcup_{i \geq 0} R_{i}
$$

where the subset $\bigcup_{i \geq 0} R_{i}$ is meager, hence also rar. Hence,

$$
\bigcup_{i \geq 0} A_{i} \oplus \bigcup_{i \geq 0} U_{i}
$$

is a rare subset and

$$
\mu\left(\bigcup_{i \geq 0} A_{i}\right)=\mu\left(\bigcup_{i \geq 0} U_{i}\right) .
$$

Therefore, showing that $\mu\left(\bigcup_{i \geq 0} A_{i}\right)=\sum_{i \geq 0} \mu\left(A_{i}\right)$ reduces to showing that

$$
\mu\left(\bigcup_{i \geq 0} U_{i}\right)=\sum_{i \geq 0} \mu\left(U_{i}\right) .
$$

Since $\bigcup_{i} U_{i}=\bigvee_{k \geq 0} \bigvee_{i<k} U_{i}$, invoking continuity and modularity (hence finite additivity) of $\nu$ completes the proof. I

Remark 2.70. Lemma 2.69 is true for all Stonean spaces. This result follows from Alvarez-Manilla 2002.d, Theorem 4.4], which says that (finite in our case) continuous valuations on a regular topological space extend uniquely to regular $\tau$-smooth Borel measures. Used in 2.64, 2.71. 5.6*

We now show that our definition of hyperstonean spaces is equivalent to Dixmier's definition [1951.a, Definition 3]. This lemma is not used anywhere else and is only established to justify our terminology.

Lemma 2.71. The class of hyperstonean spaces coincides with the class of Stonean spaces such that the union of supports of all positive normal measures is everywhere dense.

Proof. Suppose $S$ is a Stonean space such that the union of supports of all positive normal measures is everywhere dense. Then for any nonempty open subset $U \subset S$ there is a positive normal measure $\mu$ on $S$ such that the support of $\mu$ is a clopen subset of $S$ that intersects $U$. By definition of a normal measure, the measure $\mu$ restricts to a normal valuation $\nu: \Omega(S) \rightarrow[0, \infty)$ such that $\nu(U) \neq 0$. Since $U \in \Omega(S) \backslash\{\emptyset\}$ is arbitrary, this proves that $\Omega(S)$ is a hyperstonean locale and therefore $S$ is a hyperstonean space.

Suppose now that $S$ is a hyperstonean space. By Remark 2.70, any normal valuation on $\Omega(S)$ yields a normal measure on $S$. Since $\Omega(S)$ is hyperstonean, any open subset of $S$ has a nonempty intersection with the support of some normal measure on $S$. This means that the union of all such supports is everywhere dense in $S$, and hence the union of supports of all positive normal measures on $S$ is everywhere dense. I

The following proposition follows immediately from our definition of hyperstonean spaces.

Proposition 2.72. The adjoint equivalence

$$
\text { Stonean } \underset{S p}{\stackrel{\theta}{S}} \text { StoneanLog }
$$

restricts to an adjoint equivalence

$$
\text { HStonean } \underset{S p}{\stackrel{\Omega}{S}} \text { HStoneanLog. }
$$


Used in $1.1 *, 1.3 * 2.0 * 2.51 * 5.15 *$

Proposition 2.73. (Hyperstonean duality.) The adjoint equivalence

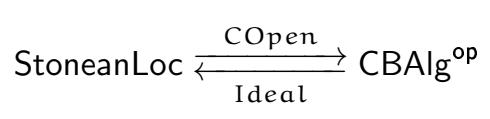

restricts to an adjoint equivalence

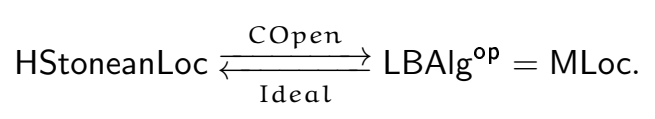

Used in $1.1 * 1.3 * 2.0 * 2.51 *$

Proof. It suffices to show that the functor COpen sends hyperstonean locales to localizable Boolean algebras and the functor Ideal sends localizable Boolean algebras to hyperstonean locales. Both claims follow immediately from Lemma 2.58. I

Remark 2.74. Continuing Remark 2.50, for a hyperstonean locale $H$ we have a canonical dense inclusion of locales

$$
\text { COpen }(H) \rightarrow H
$$

Equivalently, for a measurable locale $L$ we have a canonical dense inclusion

$$
L \rightarrow \operatorname{Ideal}(L)
$$

The inclusions exhibit their codomain, a hyperstonean locale, as the hyperstonean compactification of the domain, a measurable locale. Furthermore, the domain is the smallest dense sublocale of the codomain. The opens of the domain bijectively map to the clopens of the codomains.

\section{Duality between measurable locales and commutative von Neumann algebras}

This section constructs in Theorem 3.20 an adjoint equivalence of categories

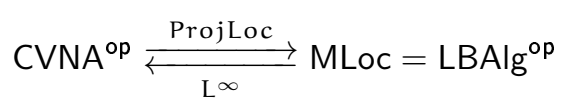

of measurable locales and commutative von Neumann algebras as the opposite adjoint equivalence of

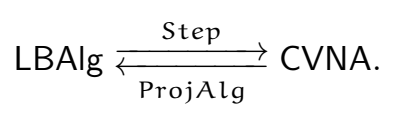

Here ProjLoc sends a commutative von Neumann algebra to its localizable Boolean algebra of projections, whereas $L^{\infty}$ sends a localizable Boolean algebra corresponding to a measurable locale $L$ to the commutative von Neumann algebra of bounded maps of locales $L \rightarrow \mathbf{C}$, where $\mathbf{C}$ denotes the usual locale of complex numbers. The essential surjectivity of ProjLoc is implied by the results of Segal $1951 . c$.

\subsection{Commutative von Neumann algebras Used in}

This section recalls the necessary definitions and facts from the theory of von Neumann algebras. Although we try to give a reasonably detailed account, our exposition is not self-contained, and we refer the reader to Sakai's book 1971.b for more information.

All rings are unital and all homomorphisms of rings preserve units. In particular, we require $\mathrm{C}^{*}$-algebras to be unital, since we do not need the nonunital ones. We use $\mathbf{C}$ as our ring of coefficients throughout this paper. (Real $\mathrm{C}^{*}$-algebras and real von Neumann algebras can be most easily defined and treated through the complexification functor: $A$ is a real $\mathrm{C}^{*}$-algebra if $A \otimes_{\mathbf{R}} \mathbf{C}$ is a complex $\mathrm{C}^{*}$-algebra, and similarly for real von Neumann algebras.) 
Definition 3.2. A $C^{*}$-algebra is a complex unital algebra $A$ equipped with a complex-antilinear map $*: A \rightarrow$ $A$ and a complete norm $\|-\|: A \rightarrow[0, \infty)$ such that $\|1\| \leq 1,\|x y\| \leq\|x\| \cdot\|y\|, 1^{*}=1,(x y)^{*}=y^{*} x^{*}$, $\left\|x^{*}\right\|=\|x\|,\left\|x^{*} x\right\|=\left\|x^{*}\right\| \cdot\|x\|$. A $C^{*}$-homomorphism $f: A \rightarrow A^{\prime}$ of C $^{*}$-algebras is a unital homomorphism of complex algebras such that $f\left(x^{*}\right)=f(x)^{*}$. (Such homomorphisms are automatically contractive: $\|f(x)\| \leq$

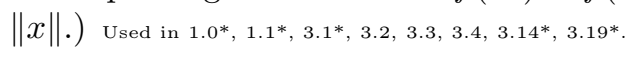

Definition 3.3. A von Neumann algebra (alias $W^{*}$-algebra) is a $\mathrm{C}^{*}$-algebra $A$ that admits a predual, i.e., a complex Banach space $A_{*}$ such that there is a (necessarily isometric) isomorphism $\int: A \rightarrow\left(A_{*}\right)^{*}$ of complex Banach spaces. A morphism of von Neumann algebras (alias normal *-homomorphism or $W^{*}$ homomorphism) is a $\mathrm{C}^{*}$-homomorphism of $\mathrm{C}^{*}$-algebras $f: A \rightarrow A^{\prime}$ that admits a predual, which is given by preduals $\left(A_{*}, \int\right)$ and $\left(A_{*}^{\prime}, \int^{\prime}\right)$ for $A$ and $A^{\prime}$ respectively, together with a (necessarily contractive) map of Banach spaces $f_{*}: A_{*}^{\prime} \rightarrow A_{*}$ such that the following square commutes:

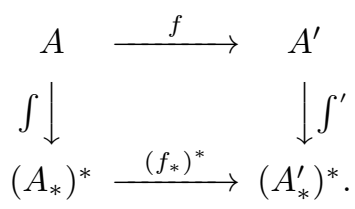

The category of von Neumann algebras is denoted by VNA. The category of commutative von Neumann

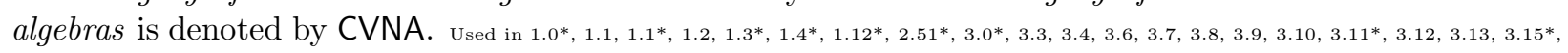
3.19. 3.18 3.19* $3.22,4.23$.

Remark 3.4. If $\left(A, \int\right)$ and $\left(A^{\prime}, \int^{\prime}\right)$ are preduals of a $\mathrm{C}^{*}$-algebra $A$, then by Sakai's theorem 1956] there is a unique (necessarily isometric) isomorphism $g: A \rightarrow A^{\prime}$ such that $\int=g^{*} \circ \int^{\prime}$. Thus all preduals of $A$ are isomorphic via a unique isomorphism, which allows us to talk about the predual of $A$. For a predual $\left(A, \int\right)$ of $A$ the isometric isomorphism $\int: A \rightarrow\left(A_{*}\right)^{*}$ endows $A$ with a topology transferred from the weak topology on $\left(A_{*}\right)^{*}$. This topology on $A$ is independent of the choice of $\left(A, \int\right)$ and is known as the ultraweak topology $(\sigma$-topology in Sakai's book [1971.b] $)$. Taking the dual of the isomorphism $\int$ with these topologies, we get an isomorphism $A_{*} \rightarrow A^{*}$, i.e., $A_{*}$ is the ultraweak dual of $A$. Likewise, given a morphism of von Neumann algebras $f: A \rightarrow A^{\prime}$, we get a commutative square

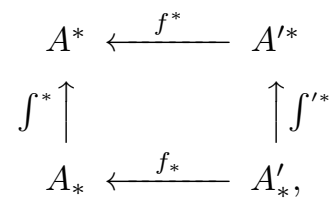

where both vertical maps are isomorphism. Thus, taking the dual in the ultraweak topology yields a functor, the predual functor, which is denoted by

$$
(-)_{*}: \mathrm{VNA}^{\mathrm{pe}} \rightarrow \text { Banach }
$$

Composing (-) with the dual Banach space functor

$$
(-)^{*}: \text { Banach } 0 \text { } \rightarrow \text { Banach }
$$

yields a functor

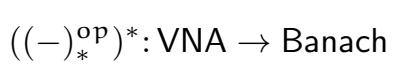

and $\int$ is a natural isomorphism

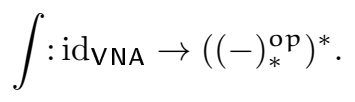

Used in 3.9 . 


\subsection{Functors between commutative von Neumann algebras and measurable locales}

Definition 3.6. For a von Neumann algebra $A$ we define

$$
A^{\geq 0}=A^{*} A=\left\{a^{*} a \mid a \in A\right\} .
$$

If $\int: A \rightarrow\left(A_{*}\right)^{*}$ is the predual isomorphism for a von Neumann algebra $A$, then we define

$$
A_{*}^{\geq 0}=\left\{\mu \in A_{*} \mid\left(\int a\right)(\mu) \geq 0 \text { for all } a \in A^{\geq 0}\right\} .
$$

Remark 3.7. A positive linear functional $A \rightarrow \mathbf{C}$ on a von Neumann algebra $A$ is ultraweakly continuous if and only if it is a normal functional, i.e., preserves suprema of bounded directed subsets. Such functionals are in bijection with elements of $A_{*}^{\geq 0}$. (Sakai [1971.b, Theorem 1.13.2].) Any element $\mu \in A_{*}$ is a complex linear combination of at most four elements of $A_{*}^{>0}$. (Sakai [1971.b, Theorem 1.14.3].) Used in [.8. . [.9.4.

Proposition 3.8. For any commutative von Neumann algebra A, its Boolean algebra of projections

$$
\operatorname{Proj} \operatorname{Alg}(A)=\left\{x \in A \mid x^{*}=x, x^{2}=x\right\}
$$

equipped with the induced operations of addition $a \oplus b=(a-b)^{2}$ and multiplication is a complete Boolean

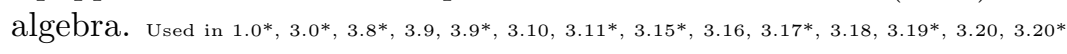

Proof. Idempotents in any commutative unital ring form a Boolean algebra with respect to the operations $a \wedge b=a b$ and $a \vee b=a+b-a b$. Self-adjoint idempotents are preserved under these operations, so they also form a Boolean algebra. The Boolean algebra $\operatorname{ProjAlg}(A)$ is complete: if $S \subset \operatorname{ProjAlg}(A)$ is an arbitrary directed subset of $\operatorname{ProjAlg}(A)$, then for any $\mu \in A_{*}^{\geq 0}$ the directed subset $\mu_{!}(S) \subset[0, \infty)$ is bounded by $\mu(1)$, therefore $\lim _{s \in S} \mu(s)$ exists for all $\mu \in A_{*}^{\geq 0}$. Since any element $\mu \in A_{*}$ is a complex linear combination of at most four elements of $A_{*}^{\geq 0}$ (Remark 3.7), we deduce that $\lim _{s \in S} \mu(s)$ exists for all $\mu \in A_{*}$, which by the definition of the ultraweak topology on $A$ means that $\lim _{s \in S} s$ exists and by continuity of the maps $a \mapsto a^{2}-a$ and $a \mapsto a-a^{*}$ must be a projection itself.

Proposition 3.9. For any commutative von Neumann algebra $A$, restriction along the inclusion of sets $\operatorname{ProjAlg}(A) \rightarrow A$ establishes a bijection from $A_{*}$ to the vector space of complex continuous valuations on $\operatorname{ProjAlg}(A)$. Used in $1.24,3.11$, 3.194

Proof. Given $\mu \in A_{*}$, we show that its restriction $\nu$ to $\operatorname{ProjAlg}(A)$ is a complex continuous valuation on $\operatorname{ProjAlg}(A)$. As established in Proposition 3.8, the Boolean algebra ProjAlg $(A)$ is complete. The properties $\nu(0)=0$ and $\nu(x)+\nu(y)=\nu(x \vee y)+\nu(x \wedge y)$ follow from the linearity of $\mu$. To show that $\nu\left(\sup _{x \in I} x\right)=\lim _{x \in I} \nu(x)$ for any directed subset $I \subset L$, it suffices to observe that $\sup _{x \in I} x=\lim _{x \in I} x$, where the limit is taken in the ultraweak topology (Sakai 1971.b, Lemma 1.7.4]).

Given a complex continuous valuation $\nu$ on $\operatorname{Proj} A l d(A)$, we show that it admits a unique extension to an ultraweakly continuous map $A \rightarrow \mathbf{C}$. By the Hahn-Jordan decomposition of normal valuations (Lemma 2.59), it suffices to treat the positive case. First, since $\nu$ is a valuation, it admits a unique extension to a linear functional on the linear span of $\operatorname{ProjAlg}(A)$ inside $A$. Secondly, since $\nu$ is a continuous valuation, the associated linear functional $f$ is a normal functional (Remark 3.7), hence also ultraweakly continuous by Remark 3.7. Finally, the linear span of all projections is ultraweakly dense in $A$, so $f$ extends uniquely to an ultraweakly continuous functional $A \rightarrow \mathbf{C}$.

Definition 3.10. The projection locale functor for commutative von Neumann algebras is a functor

$$
\text { ProjLoc: CVNA } \rightarrow \text { MLog }=\text { LBAIg }
$$

defined as the opposite functor of

$$
\text { ProjAlg: CVNA } \rightarrow \text { LBAlg }
$$


that sends a commutative von Neumann algebra $A$ to its Boolean algebra of projections (defined in Proposition 3.8), which is localizable. A morphism $f: A \rightarrow A^{\prime}$ is sent to its restriction $\operatorname{ProjAlg}(f): \operatorname{ProjAlg}(A) \rightarrow$

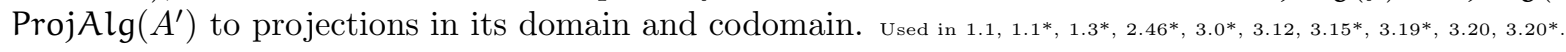

Lemma 3.11. This definition is correct.

Proof. The Boolean algebra ProjAlg $(A)$ is complete by Proposition 3.8. It is also localizable: given $m \in$ $\operatorname{ProjAlg}(A)$ such that $m \neq 0$, its image under the isomorphism $\int: A \rightarrow\left(A_{*}\right)^{*}$ is a nonzero element $\kappa \in\left(A_{*}\right)^{*}$, meaning there is $\nu \in A_{*}$ such that $\kappa(\nu)=\nu(m) \neq 0$. By decomposing $\nu$ as a complex linear combination of at most four elements of $A_{*}^{\geq 0}$, we can assume $\nu \geq 0$ and $\nu(m) \neq 0$. By Proposition 3.9, $\nu$ is a continuous valuation on $\operatorname{Proj} A l g(A)$, so $A$ is localizable by Lemma 2.53.

For any normal *-homomorphism $f: A \rightarrow A^{\prime}$ the map $\operatorname{ProjAlg}(f): \operatorname{ProjAlg}(A) \rightarrow \operatorname{ProjAlg}\left(A^{\prime}\right)$ is a homomorphism of Boolean algebras because $f\left(x^{*}\right)=f(x)^{*}$ and $f\left(x^{2}\right)=f(x)^{2}$. If $S \subset \operatorname{ProjAlg}(A)$ is a directed subset, then for any $\mu \in\left(A_{*}^{\prime}\right)^{\geq 0}$ we have $\mu(\sup f(S))=\sup \mu(f(S))=\mu(f(\sup S))$ because $\mu$ and $\mu \circ f$ preserve suprema by virtue of being elements of $\left(A_{*}^{\prime}\right)^{\geq 0}$ respectively $A_{*}^{\geq 0}$. Since $\mu \in\left(A_{*}^{\prime}\right)^{\geq 0}$ is arbitrary, this shows that $\sup f(S)=f(\sup S)$, so $f$ is a complete homomorphism. I

Remark 3.12. The composition

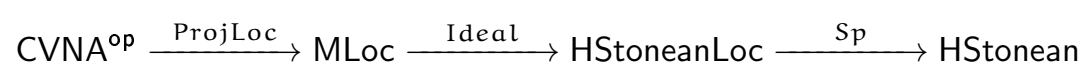

is the Gelfand spectrum functor restricted to the category of commutative von Neumann algebras, as observed

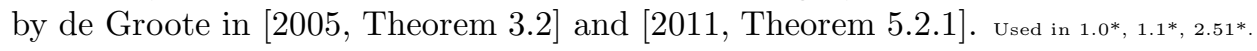

Definition 3.13. The functor

$$
\mathrm{L}^{\infty}: \mathrm{MLog} \rightarrow \text { CVNA }
$$

sends a measurable locale $L$ to the commutative von Neumann algebra of bounded (meaning factoring through some open ball) maps of locales $L \rightarrow \mathbf{C}$, where $\mathbf{C}$ denotes the usual locale of complex numbers. The functor $L^{\infty}$ sends a map of measurable locales $f: L \rightarrow L^{\prime}$ to the morphism of von Neumann algebras $\mathrm{L}^{\infty}\left(L^{\prime}\right) \rightarrow \mathrm{L}^{\infty}(L)$ that sends $h \in \mathrm{L}^{\infty}\left(L^{\prime}\right)\left(h: L^{\prime} \rightarrow \mathbf{C}\right)$ to $h \circ f \in \mathrm{L}^{\infty}(L)$. The opposite functor is denoted by

$$
\text { Step }=\left(\mathrm{L}^{\infty}\right) \text { op: LBAlg } \rightarrow \text { CVNA. }
$$

Used in $3.0^{*}, 3.14 * 3.15^{*}, 3.17,3.17,3.18,3.19 * 3.29,3.20$.

Lemma 3.14. This definition is correct. Used in 3.19 .

Proof. $\mathrm{L}^{\infty}(L)$ is a commutative complex $\mathrm{C}^{*}$-algebra because $\mathbf{C}$ is. Completeness follows from the existence of a predual shown below, so does not require a separate proof, and the other properties are of purely algebraic nature.

Below, we construct the predual in the real setting and then complexify by tensoring with $\mathbf{C}$ over $\mathbf{R}$. We set the real predual $\mathrm{L}^{\infty}(L)_{*}$ to the normed vector space $\mathrm{L}^{1}(L)$ of real continuous valuations on $L$, equipped with the variation norm defined in Lemma 2.59.

Consider the linear span of characteristic maps $\chi_{m}: L \rightarrow \mathbf{C}$ for all $m \in L$. We refer to its elements as step maps. Real step maps are order-dense in the real part of $\mathrm{L}^{\infty}(L)$ because any real $f \in \mathrm{L}^{\infty}(L)$ is the supremum of step functions given by finite subsums of $\sum_{k \in \mathbf{Z}} k / n \cdot \chi_{f^{*}[k / n,(k+1) / n)}$ as $n \rightarrow \infty$. Thus, $\mathrm{L}^{\infty}(L)$ is the Dedekind completion of the Archimedean vector lattice of real step maps. This justifies the notation Step for the functor $\left(\mathrm{L}^{\infty}\right)$ op.

Consider the linear span of characteristic functionals $\psi_{m}: \mathrm{L}^{1}(L) \rightarrow \mathbf{C}(\mu \mapsto \mu(m))$ for all $m \in L$. We refer to its elements as step functionals. Real step functionals are order-dense in the real part of $\left(\mathrm{L}^{1}(L)\right)^{*}$ because any real $F \in\left(\mathrm{L}^{1}(L)\right)^{*}$ is the supremum of step functionals given by finite subsums of $\sum_{k \in \mathbf{Z}} k / n \cdot \psi_{G_{(k+1) / n}-G_{k / n}}$, where $G_{\alpha} \in L$ for $\alpha \in \mathbf{R}$ is defined as the supremum of all $p \in L$ such that $F(\mu(p \wedge-)) \leq \alpha \mu(p)$ for all $\mu \in \mathrm{L}^{1}(L)$. Indeed, if we fix some $\mu \in \mathrm{L}^{1}(L)$, then

$$
F(\mu)-\sum_{k \in \mathbf{Z}} k / n \cdot \psi_{G_{(k+1) / n}-G_{k / n}}(\mu)=\sum_{k \in \mathbf{Z}} F\left(\mu\left(\left(G_{(k+1) / n}-G_{k / n}\right) \wedge-\right)\right)-\sum_{k \in \mathbf{Z}} k / n \cdot \mu\left(G_{(k+1) / n}-G_{k / n}\right)=A,
$$


where

$$
k / n \cdot \mu\left(G_{(k+1) / n}-G_{k / n}\right) \leq F\left(\mu\left(\left(G_{(k+1) / n}-G_{k / n}\right) \wedge-\right)\right) \leq(k+1) / n \cdot \mu\left(G_{(k+1) / n}-G_{k / n}\right),
$$

so $A \geq 0$ and

$$
A \leq \sum_{k \in \mathbf{Z}} 1 / n \cdot \mu\left(G_{(k+1) / n}-G_{k / n}\right)=\mu(1) / n
$$

Thus, $A \rightarrow 0$ as $n \rightarrow \infty$, establishing the claim. Hence, $\left(\mathrm{L}^{1}(L)\right)^{*}$ is the Dedekind completion of the Archimedean vector lattice of real step functionals.

Consider the map that sends $\chi_{m}$ to $\psi_{m}$ for any $m \in L$. This map extends by linearity to an isomorphism of Archimedean vector lattices from step functions to step functionals. Indeed, the resulting map $\zeta$ is surjective by construction. For injectivity, observe first that $\psi_{m} \neq 0$ whenever $m \neq 0$. Furthermore, an arbitrary linear combination of $\psi_{m}$ can be analyzed by restricting separately to each possible meet of elements of the form $m$ and $\neg m$ participating in the linear combination, after which both linear combinations take the form $\alpha \chi_{m}$ respectively $\alpha \psi_{m}$ for some $m \in L$. Thus, the map $\zeta$ is an isomorphism and induces an isomorphism

$$
\int_{L}: \mathrm{L}^{\infty}(L) \rightarrow\left(\mathrm{L}^{1}(L)\right)^{*}
$$

between the Dedekind completions of these Archimedean vector lattices, which shows that $\mathrm{L}^{1}(L)$ is the predual of $\mathrm{L}^{\infty}(L)$. This is the localic analogue of the Lebesgue integral, traditionally denoted by $\int_{L} f \mathrm{~d} \mu$ instead of $\int_{L}(f)(\mu)$.

Finally, given a morphism of measurable locales $f: L \rightarrow L^{\prime}$, we construct a predual of the induced $\mathrm{C}^{*}$ homomorphism of $\mathrm{C}^{*}$-algebras $\mathrm{L}^{\infty}\left(L^{\prime}\right) \rightarrow \mathrm{L}^{\infty}(L)$, which has to be a map of the form $\mathrm{L}^{1}(f): \mathrm{L}^{1}(L) \rightarrow \mathrm{L}^{1}\left(L^{\prime}\right)$. This is precisely the pushforward map on continuous valuations: given $\mu \in \mathrm{L}^{1}(L)$, we set $\mathrm{L}^{1}(f)(\mu)\left(m^{\prime}\right)=$ $\mu\left(f^{*}\left(m^{\prime}\right)\right)$. This is a contractive map of Banach spaces, and to verify the predual property, it suffices to verify the commutativity of the diagram

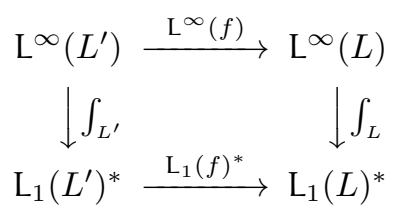

on all characteristic maps $\chi_{m^{\prime}}$, where $m^{\prime} \in L^{\prime}$. Indeed,

$$
\mathrm{L}_{1}(f)^{*}\left(\int_{L^{\prime}}\left(\chi_{m^{\prime}}\right)\right)=\mathrm{L}_{1}(f)^{*}\left(\mu^{\prime} \mapsto \mu^{\prime}\left(m^{\prime}\right)\right)=\left(\mu \mapsto \mu\left(f^{*}\left(m^{\prime}\right)\right)\right)
$$

whereas

$$
\int_{L} \mathrm{~L}^{\infty}(f)\left(\chi_{m^{\prime}}\right)=\int_{L} \chi_{f^{*}\left(m^{\prime}\right)}=\left(\mu \mapsto \mu\left(f^{*}\left(m^{\prime}\right)\right)\right)
$$

so both values coincide.

\subsection{Equivalence of commutative von Neumann algebras and measurable locales}

In this section, we construct the counit and unit of the adjunction

$$
\text { CVNA }
$$

by constructing the unit $\chi$ and counit $\mu$ of the opposite adjunction

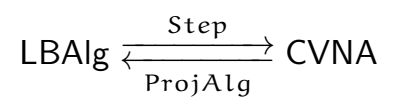

and prove that they are isomorphisms and satisfy the triangle identities. 
Definition 3.16. The natural isomorphism

$$
\chi: \mathrm{id} \text { LBAlg } \rightarrow \text { ProjAlg Step }
$$

sends a localizable Boolean algebra $L$ to the map of localizable Boolean algebras $\chi_{L}: L \rightarrow$ ProjAlg $($ Step $(L))$ that sends $m \in L$ to the projection $\chi_{m}$ in the von Neumann algebra Step $(L)$ given by the characteristic map $\chi_{m}$ of $m$.

Lemma 3.17. This definition is correct.

Proof. We have $\chi_{m m^{\prime}}=\chi_{m} \chi_{m^{\prime}}, \chi_{1}=1, \chi_{m \vee m^{\prime}}=\chi_{m} \vee \chi_{m^{\prime}}$, and $\chi_{0}=0$. Thus, $\chi_{L}$ is a homomorphism of lattices that preserves 0 and 1 , hence also a homomorphism of Boolean algebras. The map $\chi_{L}$ is injective because different $m \in L$ yield different characteristic maps. It is surjective because any map of locales $f: L \rightarrow$ C such that $f^{2}=f$ and $f^{*}=f$ necessarily factors through the map of locales given by the inclusion of the discrete locale on $\{0,1\}$ into $\mathbf{C}$. Since Boolean algebras form a variety of algebras, bijective homomorphisms of Boolean algebras are isomorphisms of Boolean algebras, hence also complete isomorphisms of complete Boolean algebras.

The naturality condition is satisfied because the following square commutes for any morphism of localizable Boolean algebras $h: L^{\prime} \rightarrow L:$

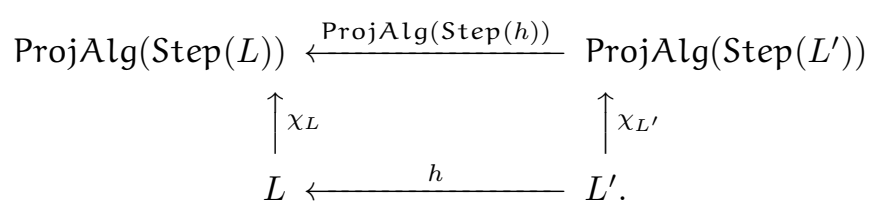

Indeed, evaluating on an arbitrary $m^{\prime} \in L^{\prime}$ yields

$$
\chi_{L}\left(h\left(m^{\prime}\right)\right)=\chi_{h\left(m^{\prime}\right)}
$$

and

$$
\operatorname{ProjAlg}(\operatorname{Step}(h))\left(\chi_{L^{\prime}}\left(m^{\prime}\right)\right)=\operatorname{ProjAlg}(\text { Step }(h))\left(\chi_{m^{\prime}}\right)=\text { Step }(h)\left(\chi_{m^{\prime}}\right)=\chi_{h\left(m^{\prime}\right)} .
$$

This establishes the naturality property and completes the proof.

Definition 3.18. The natural isomorphism

$$
\mu: \text { Step } \circ \text { ProjAlg } \rightarrow \text { id CVNA }
$$

sends a commutative von Neumann algebra $A$ to the morphism of von Neumann algebras

$$
\text { Step } \operatorname{ProjAlg}(A)) \rightarrow A
$$

whose value on the characteristic map $\chi_{m}$ of any projection $m \in A$ equals $m$. (The inverse isomorphism essentially encodes the spectral theorem for bounded operators: it sends an element $a \in A$ to the map of locales ProjAlg $(A) \rightarrow \mathbf{C}$ whose inverse image map sends an open subset of $\mathbf{C}$ to the corresponding spectral projection of $a$.)

Lemma 3.19. This definition is correct.

Proof. We work with the real parts of $\operatorname{Step}(\operatorname{ProjAlg}(A))$ and $A$. The canonical inclusion $\operatorname{ProjAlg}(A) \rightarrow A$ induces a ${ }^{*}$-homomorphism to $A$ from the linear span of all characteristic maps $\chi_{m}: \operatorname{ProjLod}(A) \rightarrow \mathbf{R}$, where

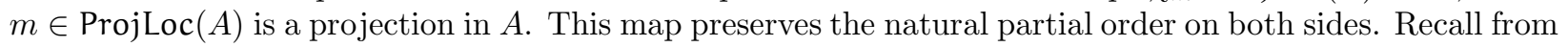
the proof of Lemma 3.14 that this linear span is order-dense in the real part of Step $(\operatorname{ProjAlg}(A))$. Therefore, we have a canonical order-preserving map $\mu_{A}: \operatorname{Step}(\operatorname{ProjAlg}(A)) \rightarrow A$, which is a $\mathrm{C}^{*}$-homomorphism by continuity.

To show that $\mu_{A}$ is a morphism of von Neumann algebras, we construct its predua as a morphism of Banach spaces

$$
A_{*} \rightarrow \mathrm{L}^{1}(\operatorname{ProjAlg}(A)),
$$


where $\mathrm{L}^{1}$ denotes the vector space of real continuous valuations of $\operatorname{ProjAlg}(A)$ as in the proof of Lemma 3.14. Indeed, Proposition 3.9 constructs such a map and proves that it is an isomorphism. Therefore, its dual map, given by the bottom map in the following square diagram, is also an isomorphism. The predual square

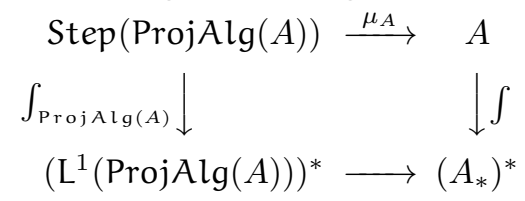

commutes because the two compositions coincide on step maps $\chi_{m}(m \in \operatorname{ProjAlg}(A))$ by construction, therefore they coincide on their linear span and its order-closure. Thus, $\mu_{A}$ is an isomorphism of von Neumann algebras because the other three maps in the square are isomorphisms.

The naturality condition is satisfied because the following square commutes for any homomorphism $h: A \rightarrow A^{\prime}$ of commutative von Neumann algebras:

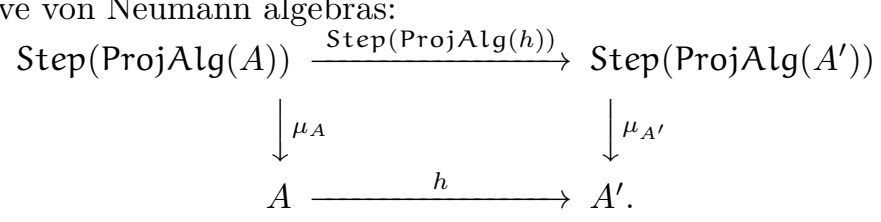

It suffices to verify commutativity when evaluated on projections in Step $(\operatorname{ProjAlg}(A))$. Indeed, given such a projection $\chi_{m}$ for some $m \in \operatorname{ProjAlg}(A)$, we have

$$
h\left(\mu_{A}\left(\chi_{m}\right)\right)=h(m)
$$

and

$$
\mu_{A^{\prime}}\left(\operatorname{Step}(\operatorname{ProjAlg}(h))\left(\chi_{m}\right)\right)=\mu_{A^{\prime}}\left(\chi_{m} \circ \operatorname{ProjAlg}(h)\right)=\mu_{A^{\prime}}\left(\chi_{h(m)}\right)=h(m),
$$

which completes the proof.

Assembling all the results of this section, we obtain the following result. While several parts of this result were known before (e.g., the essential surjectivity of ProjLod falls out of results due to Segal [1951.c]), there does not appear to be a published source that assembles the individual ingredients in a single proof.

Theorem 3.20. The functors

$$
\text { Step: LBAIg } \rightarrow \text { CVNA }
$$

and

together with natural isomorphisms

$$
\text { ProjAlg: CVNA } \rightarrow \text { LBAlg }
$$

$$
\chi: \mathrm{id} \text { LBAlg } \rightarrow \text { ProjAlg } \circ \text { Step }
$$

and

$$
\mu: \text { Step } \circ \text { ProjAlg } \rightarrow \text { id CVNA }
$$

form an adjoint equivalence of categories. Therefore, the opposite adjunction

$$
\text { CVNA op } \underset{\mathrm{L}^{\infty}}{\stackrel{\text { ProjLod }}{\rightleftarrows}} \mathrm{MLod}=\text { LBAIg }
$$

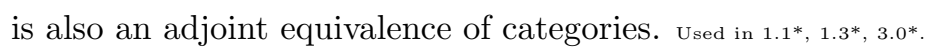

Proof. It remains to show that the exhibited equivalence is an adjoint equivalence. The two triangle identities imply one another, so it suffices to establish just one of them. To show that the composition

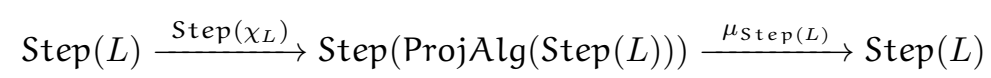

equals identity, it suffices to evaluate it on all characteristic maps $\chi_{m}$ for $m \in L$. We have

$$
\text { Step }\left(\chi_{L}\right)\left(\chi_{m}\right)=\chi_{\chi_{m}} \text {, }
$$

i.e., the characteristic map $\chi_{\chi_{m}}: \operatorname{ProjLod}\left(\mathrm{L}^{\infty}(L)\right) \rightarrow \mathbf{R}$ of the projection $\chi_{m} \in \operatorname{ProjLoc}\left(\mathrm{L}^{\infty}(L)\right)$. Then

$$
\mu \text { Step }(L)\left(\chi_{\chi_{m}}\right)=\chi_{m}
$$

which completes the proof. 


\section{Point-set measurable spaces}

This section defines the category CSLEMS in Theorem 1.1, namely, the category of compact strictly localizable enhanced measurable spaces, and explores its properties that will be necessary later.

\subsection{Enhanced measurable spaces}

Definition 4.2. A $\sigma$-algebra on a set $X$ is a collection of subsets of $X$ closed under complements and

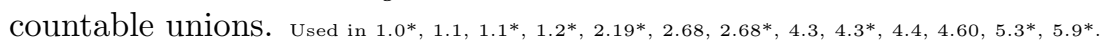

Definition 4.3. A $\sigma$-ideal $N$ of a $\sigma$-algebra $M$ on a set $X$ is a subset of $M$ closed under countable unions and passage to subsets in $M$ (meaning $A \subset B, A \in M$, and $B \in N$ imply $A \in N$ ). Used in t.0. 1.1. [.1. 2. 2.68. 2.68. $5.3 .6 .5 .6 .5,0.65,5.3 * 5.9 .5 .5 .12$

In particular, a $\sigma$-ideal of the $\sigma$-algebra $2^{X}$ consisting of all subsets of $X$ is a collection of subsets of $X$ closed under passage to subsets and countable unions.

Definition 4.4. A (complete) enhanced measurable space is a triple $(X, M, N)$, where $X$ is a set, $M$ is a $\sigma$-algebra on $X$, and $N$ is a $\sigma$-ideal of $2^{X}$ such that $N \subset M$. A measurable set is an element of $M$ and a negligible set is an element of $N$. A conegligible set is the complement of a negligible set with respect to $X$.

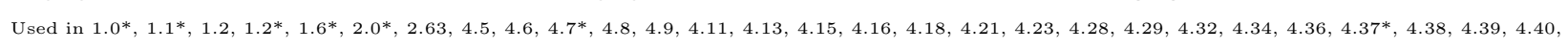

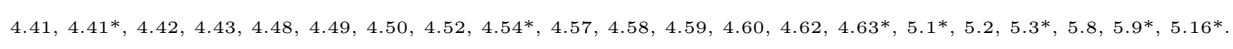

Definition 4.5. If $(X, M, N)$ is an enhanced measurable space and $m \in M$, then the induced enhanced measurable space on $m$ is the triple $\left(m, M_{m}, N_{m}\right)$, where $M_{m}=\left\{m^{\prime} \in M \mid m^{\prime} \subset m\right\}$ and $N_{m}=\{n \in N \mid$ $n \subset m\}$. Used in $1.2+, 4.28,4.38,4.41,4.61,4.65 * 5.11$.

Definition 4.6. The category PreEMS is defined as follows. Its objects are enhanced measurable spaces. Morphisms $\left(X, M_{X}, N_{X}\right) \rightarrow\left(Y, M_{Y}, N_{Y}\right)$ are premaps of enhanced measurable spaces, defined as maps of sets $f: X^{\prime} \rightarrow Y$ such that $X^{\prime} \subset X$ is a conegligible set (denoted by pdom $f$, which stands for point-set domain),

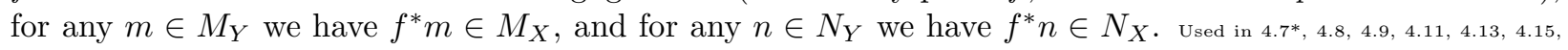

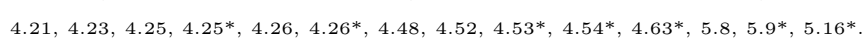

Wendt [1992, 1994.b, 1996, 1998 refers to the last property (i.e., $\left(f^{*}\right) ! N_{Y} \subset N_{X}$ ) as "measure zero reflecting".

Proposition 4.7. This definition is correct.

Proof. We define pdom $(g \circ f)=\operatorname{pdom} f \cap f^{*}(\overline{\operatorname{pdom}} g)$, which is conegligible because both factors are, the latter because $f^{*}$ preserves conegligible subsets. Morphisms are composed like maps of sets, after we restrict them to the new point-set domains. We have

$$
\begin{aligned}
\operatorname{pdom}(h \circ(g \circ f)) & =\operatorname{pdom}(g \circ f) \cap(g \circ f)^{*}(\operatorname{pdom} h) \\
& =\operatorname{pdom} f \cap f^{*}(\underline{\operatorname{pdom}} g) \cap f^{*} g^{*}(\operatorname{pdom} h)
\end{aligned}
$$

and

$$
\begin{aligned}
\operatorname{pdom}((h \circ g) \circ f) & =\underline{\operatorname{pdom}} f \cap f^{*}(\underline{\operatorname{pdom}}(h \circ g)) \\
& =\underline{\operatorname{pdom}} f \cap f^{*}\left(\underline{\operatorname{pdom}} g \cap g^{*}(\text { pdom } h)\right) \\
& =\text { pdom } f \cap f^{*}(\underline{\operatorname{pdom}} g) \cap f^{*} g^{*}(\text { pdom } h),
\end{aligned}
$$

so composition is indeed associative. The point-set composition is a premap because $(g \circ f)^{*}=f^{*} \circ g^{*}$. I

Remark 4.8. One could drop the completeness condition and define a category PrelEMS (I for incomplete) of not necessarily complete enhanced measurable spaces. Its objects are triples $(X, M, N)$, where $X$ and $M$ are as above and $N$ is a $\sigma$-ideal of $M$. A morphism $\left(X, M_{X}, N_{X}\right) \rightarrow\left(Y, M_{Y}, N_{Y}\right)$ is a map of sets $f: X^{\prime} \rightarrow Y$ such that $X^{\prime}$ is conegligible (now meaning that $X \backslash X^{\prime}$ is a subset of some $n \in N_{X}$ ), for any $m_{Y} \in M_{Y}$ we have $f^{*}\left(m_{Y}\right) \oplus m_{X} \subset n_{X}$ for some $m_{X} \in M_{X}$ and $n_{X} \in N_{X}$, and for any $n_{Y} \in N_{Y}$ we have $f^{*}\left(n_{Y}\right) \subset n_{X}$ for some $n_{X} \in N_{X}$. The inclusion functor $\iota:$ PreEMS $\rightarrow$ PrelEMS exhibits PreEMS as a full subcategory of PrelEMS, with its image consisting precisely of complete objects, i.e., objects $(X, M, N)$ for which $N$ is a $\sigma$-ideal of $2^{X}$, meaning $N$ is closed under passage to subsets in $X$. The completion functor 
$\mathrm{C}:$ PrelEMS $\rightarrow$ PreEMS sends a space $\left(X, M_{X}, N_{X}\right)$ to $\left(X, M_{X}^{\prime}, N_{X}^{\prime}\right)$, where $n^{\prime} \in N_{X}^{\prime}$ if there is $n \in N_{X}$ such that $n^{\prime} \subset n$ and $m^{\prime} \in M_{X}^{\prime}$ if there is $m \in M_{X}$ such that $m^{\prime} \oplus m \in N_{X}^{\prime}$. On morphisms, $C$ retains the underlying map of sets. The functor $\mathrm{C}$ is fully faithful. For any $\hat{X}=\left(X, M_{X}, N_{X}\right)$ the identity map of sets $X \rightarrow X$ yields isomorphisms $\hat{X} \rightarrow \mathrm{C} \hat{X} \rightarrow \hat{X}$ in PrelEMS. Thus, the inclusion $\iota:$ PreEMS $\rightarrow$ PrelEMS together with the completion functor $\mathrm{C}:$ PrelEMS $\rightarrow$ PreEMS is an adjoint equivalence of categories. Hence, there is no benefit for us to consider noncomplete spaces. Used in 1.1 .1 .7 .6 .6 .5$.

Remark 4.9. We could require $\operatorname{pdom} f=X$ (i.e., $X^{\prime}=X$ ), which would work in most of the paper. However, a crucial point where it is absolutely necessary to allow pdom $f \neq X$ is Proposition 5.16, where we construct a morphism from an enhanced measurable space $(X, M, N)$ to the Stone spectrum of the localizable Boolean algebra $M / N$. If $X \in N$, then $M / N=0$ and its Stone spectrum is empty, so the only map of sets into it must have pdom $f=\emptyset$. Otherwise we would have to manually exclude the case $X \in N, X \neq \emptyset$ from enhanced measurable spaces, which would create even more problems.

\subsection{Equality almost everywhere}

Definition 4.11. The category StrictEMS of enhanced measurable spaces and strict maps is the quotient of the category PreEMS by the equivalence relation $\sim$ of equality almost everywhere, for which

$$
f \sim f^{\prime}:\left(X, M_{X}, N_{X}\right) \rightarrow\left(Y, M_{Y}, N_{Y}\right)
$$

if $\left\{x \in X \mid f(x) \neq f^{\prime}(x)\right\} \in N_{X}$. (If one of $f(x)$ or $f^{\prime}(x)$ is undefined, then the other one must be defined in order for $f(x) \neq f^{\prime}(x)$ to hold. The easiest way to think about this is to assume $f(x)=*$ for some $* \notin Y$

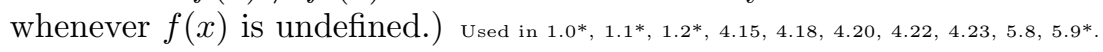

Proposition 4.12. This definition is correct.

Proof. Throughout this proof we follow the convention that $f(x)=* \notin Y$ whenever $f(x)$ is undefined. Observe that $f \sim f^{\prime}$ is indeed an equivalence relation: the reflexivity and symmetry properties are obvious, and if $f \sim f^{\prime}$ and $f^{\prime} \sim f^{\prime \prime}$, then $f \sim f^{\prime \prime}$ because

$$
\left\{x \in X \mid f(x) \neq f^{\prime \prime}(x)\right\} \subset\left\{x \in X \mid f(x) \neq f^{\prime}(x)\right\} \cup\left\{x \in X \mid f^{\prime}(x) \neq f^{\prime \prime}(x)\right\} \in N_{X} .
$$

The equivalence relation is compatible with composition: if

$$
f \sim f^{\prime}:\left(X, M_{X}, N_{X}\right) \rightarrow\left(Y, M_{Y}, N_{Y}\right)
$$

and

$$
g:\left(Y, M_{Y}, N_{Y}\right) \rightarrow\left(Z, M_{Z}, N_{Z}\right),
$$

then $g \circ f \sim g \circ f^{\prime}$ because

$$
\left\{x \in X \mid g(f(x)) \neq g\left(f^{\prime}(x)\right)\right\} \subset\left\{x \in X \mid f(x) \neq f\left(x^{\prime}\right)\right\} \in N_{X} .
$$

Likewise, if $f:\left(X, M_{X}, N_{X}\right) \rightarrow\left(Y, M_{Y}, N_{Y}\right)$ and $g \sim g^{\prime}:\left(Y, M_{Y}, N_{Y}\right) \rightarrow\left(Z, M_{Z}, N_{Z}\right)$, then $g \circ f \sim g^{\prime} \circ f$ because

$$
\left\{x \in X \mid g(f(x)) \neq g^{\prime}(f(x))\right\} \subset f^{*}\left\{y \in Y \mid g(y) \neq g^{\prime}(y)\right\} \in N .
$$

Thus, the quotient category exists. I

Definition 4.13. The category EMS of enhanced measurable spaces is the quotient of the category PreEMS by the equivalence relation $\approx$ of weak equality almost everywhere, for which

$$
f \approx f^{\prime}:\left(X, M_{X}, N_{X}\right) \rightarrow\left(Y, M_{Y}, N_{Y}\right)
$$

if for any $m \in M_{Y}$ we have $\chi_{m} \circ f \sim \chi_{m} \circ f^{\prime}$, where

$$
\chi_{m}:\left(Y, M_{Y}, N_{Y}\right) \rightarrow\left(\{0,1\}, 2^{\{0,1\}},\{\emptyset\}\right)
$$


is the characteristic map of $m$ : we have $\chi_{m}(y)=1$ if and only if $y \in m$, for any $y \in Y$. Equivalently, $f \approx f^{\prime}$

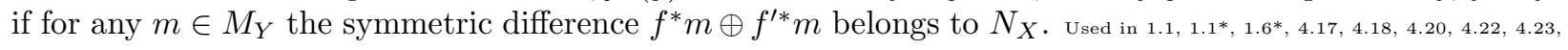

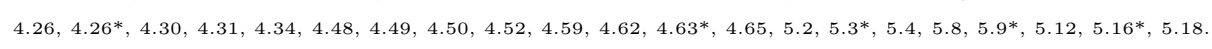

Proposition 4.14. This definition is correct.

Proof. Observe that $f \approx f^{\prime}$ is an equivalence relation: the reflexivity and symmetry properties are obvious, and if $f \approx f^{\prime}$ and $f^{\prime} \approx f^{\prime \prime}$, then $f \approx f^{\prime \prime}$ because $\chi_{m} \circ f \sim \chi_{m} \circ f^{\prime} \sim \chi_{m} \circ f^{\prime \prime}$ for all $m \in M_{Y}$, and we already know that $\sim$ is an equivalence relation. The equivalence relation is compatible with composition: if $f \approx f^{\prime}:\left(X, M_{X}, N_{X}\right) \rightarrow\left(Y, M_{Y}, N_{Y}\right)$ and $g:\left(Y, M_{Y}, N_{Y}\right) \rightarrow\left(Z, M_{Z}, N_{Z}\right)$, then $g \circ f \sim g \circ f^{\prime}$ because for any $m \in M_{Z}$ we have

$$
(g \circ f)^{*} m \oplus\left(g \circ f^{\prime}\right)^{*} m=f^{*}\left(g^{*} m\right) \oplus f^{\prime *}\left(g^{*} m\right) \in N_{X}
$$

since $g^{*} m \in M_{Y}$. Likewise, if $f:\left(X, M_{X}, N_{X}\right) \rightarrow\left(Y, M_{Y}, N_{Y}\right)$ and $g \approx g^{\prime}:\left(Y, M_{Y}, N_{Y}\right) \rightarrow\left(Z, M_{Z}, N_{Z}\right)$, then $g \circ f \approx g^{\prime} \circ f$ because for any $m \in M_{Z}$ we have

$$
(g \circ f)^{*} m \oplus\left(g^{\prime} \circ f\right)^{*} m=f^{*}\left(g^{*} m \oplus g^{\prime *} m\right) \in N_{X}
$$

since $g^{*} m \oplus g^{\prime *} m \in N_{Y}$. Thus, the quotient category exists.

Remark 4.15. If $Y \neq \emptyset$, any premap $f:\left(X, M_{X}, N_{X}\right) \rightarrow\left(Y, M_{Y}, N_{Y}\right)$ with pdom $f \neq X$ can be extended to a premap $f^{\prime}:\left(X, M_{X}, N_{X}\right) \rightarrow\left(Y, M_{Y}, N_{Y}\right)$ by setting $f(x)=y$ for all $x \in X \backslash$ pdom $f$, for some fixed $y \in Y$. We have $f \sim f^{\prime}$, so if $Y \neq \emptyset$, we could require premaps to be everywhere defined. However, if $Y=\emptyset$ and $X \in N_{X}$, there are no premaps $f:\left(X, M_{X}, N_{X}\right) \rightarrow\left(Y, M_{Y}, N_{Y}\right)$ with pdom $f=X$, even though the two enhanced measurable spaces are isomorphic in the category StrictEMS via the empty map of sets $\emptyset \rightarrow \emptyset$, since $\emptyset$ is conegligible in both spaces.

Definition 4.16. We define two enhanced measurable spaces: $\mathbf{C}_{\text {Borel }}=(\mathbf{C}, M \overline{\text { Borel }},\{\emptyset\})$ and $\mathbf{C}_{\text {Lebesgue }}=$ $(\mathbf{C}, M$ Lebesgue,$N$ Lebesgue $)$. Here $M$ Borel is the set of all Borel subsets of $\mathbf{C}, M$ Lebesgue is the set of all Lebesguemeasurable subsets of $\mathbf{C}$, and $N$ Lebesgue is the set of all Lebesgue-negligible subsets of $\mathbf{C}$. In particular, $M$ Lebesgu is the set of unions of elements of $M_{\text {Bore }}$ and $N_{\text {Lebesgue }}$. We define $\mathbf{R}$ Bore and $\mathbf{R}$ Lebesgue in a similar way. Used in $4.19,4.17,4.18,4.23,4.3$.

Remark 4.17. C Borel and CEebesgu are not isomorphic in EMS. As we will see later, the Boolean algebra $M / N$ is an isomorphism invariant of an object in EMS. For C Borel this Boolean algebra has atoms given by singleton subsets of $\mathbf{C}$, whereas for $\mathbf{C}$ Lebesgut this Boolean algebra is atomless.

Example 4.18. Recall that any Lebesgue-measurable function $\mathbf{C} \rightarrow \mathbf{C}$ is equal almost everywher to a Borel measurable function, so after passing to the category EMS there is no difference between the two notions, both give a morphism OLebesgue $\rightarrow$ C Bore. Morphisms C Lebesgu $\rightarrow$ CEebesgu form a proper subset of Lebesgue measurable functions. (Preimages of negligible subsets of $\mathbf{C}$ under Lebesgue measurable functions need not be negligible, as witnessed by constant functions.) Morphisms $\mathbf{C}$ Bore $\rightarrow \mathbf{C}_{\text {Borel }}$ are precisely Borel measurable functions, without any identification. There are no morphisms $\mathbf{C}$ Borel $\rightarrow$ Cebesgue because preimages of negligible subsets must be negligible, hence empty, but any singleton subset of the codomain is negligible.

\subsection{Comparison of equivalence relations on morphisms}

We now compare the two equivalence relations $\sim$ and $\approx$.

Lemma 4.20. If $f \sim f^{\prime}:\left(X, M_{X}, N_{X}\right) \rightarrow\left(Y, M_{Y}, N_{Y}\right)$, then $f \approx f^{\prime}$. In particular, EMS is a quotient of StrictEMS.

Proof. We have

$$
\left\{x \in X \mid \chi_{m}(f(x)) \neq \chi_{m}\left(f^{\prime}(x)\right)\right\} \subset\left\{x \in X \mid f(x) \neq f^{\prime}(x)\right\}
$$

for any $m \in M_{Y}$. 
Definition 4.21. An enhanced measurable space $(X, M, N)$ is countably separated if there is a countable subset $M_{s} \subset M$ such that for any distinct $x, x^{\prime} \in X$ there is $m \in M_{s}$ with $x \in m$ and $x^{\prime} \notin m$ and also for any $x \in X$ there is $m \in M_{s}$ with $x \in m$. (The last condition is nontrivial only when $X$ is a singleton and is necessary below for premaps whose point-set domain is a proper subset.) Used in $4.1 ., 4.22 .4 .23$.

Lemma 4.22. If $f \approx f^{\prime}:\left(X, M_{X}, N_{X}\right) \rightarrow\left(Y, M_{Y}, N_{Y}\right)$ and $\left(Y, M_{Y}, N_{Y}\right)$ is countably separated, then $f \sim f^{\prime}$. In particular, the quotient map StrictEMS $\rightarrow$ EMS becomes an equivalence of categories if we restrict to countably separated spaces on both sides. Used in 6.14 . 1.23 .

Proof. We have

$$
\{x \in X \mid f(x) \neq g(x)\} \subset \bigcup_{m \in M_{s}}\left(f^{*} m \oplus g^{*} m\right) \in N_{X} .
$$

Remark 4.23. Lemma 4.22 is false without the assumption of countable separability, see Fremlin 2002.d, Example 343I], which constructs a morphism $f: X \rightarrow X$ such that $f \approx \operatorname{id}_{X}$ and $\operatorname{pdom} f=X$, but $f(x) \neq x$ for all $x \in X$, where $X=\{0,1\}^{\mathbf{R}}$ is the 6 -finite enhanced measurable space given by the product of a continuum many copies of the two-point space $\{0,1\}$. In particular, the quotient functor StrictEMS $\rightarrow$ EMS is not an equivalence of categories. The condition of countable separability considered above is very restrictive: by Lemma 343E in Fremlin [2002.d] it is equivalent to the existence of a morphism of enhanced measurable spaces $f:(X, M, N) \rightarrow \mathbf{R}$ Borel whose underlying map of sets is injective. Thus, for arbitrary pnhanced measurable spaces (even if assumed to be $\sigma$-finite) the equivalence relation of equality almost everywhere is too strict: it fails to identify distinct premaps of enhanced measurable spaces that are sent to identical maps of the corresponding measurable locales or commutative von Neumann algebras by the relevant functors, since these functors factor through EMS. Used in $1.1^{*}$.

\subsection{Coproducts of enhanced measurable spaces}

Lemma 4.25. The category PreEMS admits small coproducts and the forgetful functor PreEMS $\rightarrow$ Set creates (i.e., preserves and reflects) small coproducts.

Proof. We construct the coproduct $\coprod_{i \in I}\left(X_{i}, M_{i}, N_{i}\right)$ by setting the underlying set to $Y=\coprod_{i \in I} X_{i}$ and declaring that $m \in M_{Y}$ (respectively $n \in N_{Y}$ ) if for every $i \in I$ we have $m \cap X_{i} \in M_{i}$ (respectively $n \cap X_{i} \in$ $\left.N_{i}\right)$. The injection maps $\iota_{i}: X_{i} \rightarrow \coprod_{i \in I} X_{i}$ yield morphisms in PreEMS. To show the universal property of coproducts, it suffices to observe that given a collection of morphisms $\left(f_{i}:\left(X_{i}, M_{i}, N_{i}\right) \rightarrow\left(Z, M_{Z}, N_{Z}\right)\right)_{i \in I}$ (with pdom $f_{i}=X_{i}^{\prime} \subset X_{i}$ ) in PreEMS, the induced map of sets $\left[f_{i}\right]_{i \in I}: \coprod_{i \in I} X_{i}^{\prime} \rightarrow Z$ is a morphism in PreEMS, which follows from the definition of $M_{Y}$ and $N_{Y}$. Since the forgetful functor reflects isomorphisms, reflection of coproducts is implied by preservation of coproducts. I

Lemma 4.26. The category EMS admits small coproducts and the quotient functor PreEMS $\rightarrow$ EMS preserves small coproducts.

Proof. Since PreEMS admits small coproducts, it suffices to show that given a coproduct cocone in PreEMS, its image in EMS is also a coproduct cocone. We prove the latter claim by establishing the universal property of coproducts. Existence follows from the fact that the quotient functor is full and surjective on objects. Uniqueness amounts to showing that $f \circ \iota_{i} \approx g \circ \iota_{i}$ for all $i$ implies $f \approx g$, which follows from the definition of $\approx$ and the construction of negligible sets in the coproduct.

\subsection{Measures on enhanced measurable spaces}

Definition 4.28. A (complex infinite) measure on an enhanced measurable space $(X, M, N)$ is a map $\mu: M^{\prime} \rightarrow \mathbf{C}$, where $M^{\prime} \subset M$ is an ideal of $M$ such that $N \subset M^{\prime}$, the map $\mu$ vanishes on $N$, and the ideal $M^{\prime}$ satisfies the following saturation condition: for any countable family $\left\{m_{i}\right\}_{i \in I}$ of disjoint elements of $M^{\prime}$ such that the sum $\sum_{i \in I} \mu\left(m_{i}^{\prime}\right)$ converges absolutely for any family $\left\{m_{i}^{\prime}\right\}_{i \in I}$ satisfying $m_{i}^{\prime} \subset m_{i}$ and $m_{i}^{\prime} \in M^{\prime}$, we have

$$
\bigcup_{i \in I} m_{i} \in M^{\prime}, \quad \mu\left(\bigcup_{i \in I} m_{i}\right)=\sum_{i \in I} \mu\left(m_{i}\right) .
$$

We say that $\mu$ is a 
- faithful measure if $\left.\mu\right|_{m}=0$ implies $m \in N$ for any $m \in M^{\prime}$;

- finite measure if $M^{\prime}=M$;

- semifinite measure if for any $m \in M \backslash N$ there is $m^{\prime} \in M^{\prime} \backslash N$ such that $m^{\prime} \subset m$;

- real measure (alias signed measure or charge) if $\mu$ factors through $\mathbf{R} \rightarrow \mathbf{C}$;

- positive measure if $\mu$ factors through $[0, \infty) \rightarrow \mathbf{C}$.

Here $\left.\mu\right|_{m}$ for $m \in M$ denotes the restriction of $\mu$ to $M_{m}^{\prime}=\left\{m^{\prime} \in M^{\prime} \mid m^{\prime} \subset m\right\}$, which is a measure on the induced enhanced measurable space $\left(m, M_{m}, N_{m}\right)$. Also, 0 denotes the zero measure, with $M^{\prime}=M$ and $\mu(m)=0$ for all $m \in M^{\prime}$. Two measures are equal if they have the same ideal $M^{\prime}$ and take the same values

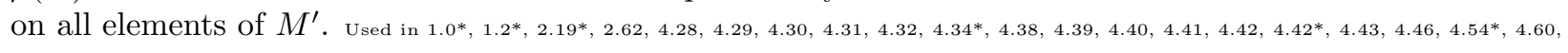

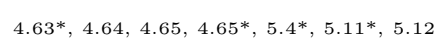

Remark 4.29. The definition of a finite measure can be simplified as follows: a finite measure on an enhanced measurable space $(X, M, N)$ is a countably additive map $M \rightarrow \mathbf{C}$ that vanishes on $N$. Countable additivity means that for any countable family $\left\{m_{i}\right\}_{i \in I}$ of disjoint elements of $M$ the sum $\sum_{i \in I} \mu\left(m_{i}\right)$ converges absolutely and $\mu\left(\bigcup_{i \in I} m_{i}\right)=\sum_{i \in I} \mu\left(m_{i}\right)$.

Remark 4.30. By the Radon-Nikodym theorem, complex semifinite measures on a localizable enhanced measurable space $(X, M, N)$ form a free module of rank 1 over the complex algebra of all morphisms (in the category EMS) of the form $(X, M, N) \rightarrow \mathbf{C}$ Bore. This necessitates the consideration of emifinite measures and not just finite measures. Some definitions of infinite signed measures found in the literature require that either the positive or negative part in the Jordan decomposition is finite. Such a convention would preclude the strong version of the Radon-Nikodym given above, which motivates our version.

Remark 4.31. The pushforward of a semifinite measure along a morphism in EMS need not be semifinite, which requires us to also consider nonsemifinite measures in the above definition.

Remark 4.32. If $\mu$ is a positive measure on an enhanced measurable space $(X, M, N)$, then setting $\mu(m)=$ $\infty$ for every $m \in M \backslash M^{\prime}$ produces a countably additive map $M \rightarrow[0, \infty]$. Thus, $(X, M, \mu)$ is a measure space in the traditional sense. Conversely, if $(X, M, \mu)$ is a complete measure space in the traditional sense, then setting $N=\{m \in M \mid \mu(m)=0\}$ and $M^{\prime}=\{m \in M \mid \mu(m) \neq \infty\}$ produces an enhanced measurable space $(X, M, N)$ together with a positive aaithful measure $\left.\mu\right|_{M^{\prime} \text {. Used in } . .39}$

\subsection{Localizable enhanced measurable spaces}

The following definition was introduced (in the nonenhanced case) by Irving Segal [1951.b, Definition 2.6]. The choice of terminology is motivated by Theorem 5.1 in the cited paper, which proves that being localizable is equivalent to the Lebesgue decomposition property: any $\sigma$-ideal $I \subset M$ is localized at some measurable set $P$ in the sense that $Q \in I$ if and only if $Q \backslash P$ is negligible.

Definition 4.34. A localizable enhanced measurable space is an enhanced measurable space $(X, M, N)$ such that the Boolean algebra $M / N$ is localizable (Definition 2.52). The full subcategory of localizable objects

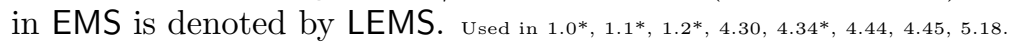

Localizable measure spaces as defined by Segal $1951 . b$ can be now identified with localizable enhanced measurable spaces equipped with a positive faithful semifinite measure.

Remark 4.35. It is useful to reformulate the existence of suprema in $M / N$ without referring to the quotient Boolean algebra. Suppose $\left\{m_{i}\right\}_{i \in I}$ is a family of elements of $M$ and $\hat{m} \in M$. We have $[\hat{m}]=\sup _{i \in I}\left[m_{i}\right]$ if and only if $m_{i} \backslash \hat{m} \in N$ for all $i \in I$ and whenever for some $\hat{m}^{\prime} \in M$ we have $m_{i} \backslash \hat{m}^{\prime} \in N$ for all $i \in I$, then $\hat{m} \hat{m}^{\prime} \in N$. The set $\hat{m}$ is also known as the essential supremum of the family $\left\{m_{i}\right\}_{i \in I}$. Used in 4.41 , 4.41 , 4.42 $4.42,4.45^{3}, 4.63^{3}, 4.65^{4}$

Remark 4.36. Dixmier 1951.a, $\S 7$ starting from Lemma 8] constructs a complete Boolean algebra $A$ whose Stone spectrum is a Stonean space $S$ in which every meager subset is are and the support of every measure is rard. The enhanced measurable space $(X, M, N)=\mathrm{TM}(S)$ is such that the Boolean algebra $M / N \cong A$ is complete and every measure on $(X, M, N)$ vanishes. Thus, the Boolean algebra $A$ is complete but not ocalizable. Therefore, we must include the requirement of existence of sufficiently many continuous valuations on $M / N$ in the definition of localizability. Segal 1951.b imposes this requirement implicitly, 
by virtue of working with a prespecified semifinite measure $m: M \rightarrow[0, \infty]$ whose class of measure 0 sets coincides with our $N$.

\subsection{Essential measures on enhanced measurable spaces}

The goal of this section is to explain the "correct" definition of a measure on an enhanced measurable space that is not $\sigma$-finite. Here "correct" means, in particular, that Proposition 4.45 holds.

Definition 4.38. An enhanced measurable space $(X, M, N)$ is $\sigma$-finite if it admits a faithful finite measure. An element $m \in M$ is $\sigma$-finite if the induced enhanced measurable space on $m$ is $\sigma$-finite. Used in $1.0 * 1.1 .+1.2 .4$.

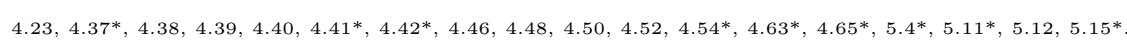

Remark 4.39. If $\mu$ is a $\sigma$-finite measure on a measurable space $(X, M)$ (in the usual sense, meaning $X$ is a countable union of elements of $M$ with a finite $\mu$-measure), then the underlying enhanced measurable space in the sense of Remark 4.32 is a $\sigma$-finite enhanced measurable space. Indeed, in this case $X$ can be presented as a countable disjoint union $\bigcup_{i>0} m_{i}$ of elements of $M$ with a finite nonzero $\mu$-measure, and setting $\nu\left(m^{\prime}\right)=\sum_{i>0} \mu\left(m^{\prime} \cap m_{i}\right) /\left(2^{i} \mu\left(m_{i}\right)\right)$ produces a finite measure on $(X, M)$ with the same class of negligible sets. Conversely, if $\mu$ is a faithful measure on the enhanced measurable $\operatorname{space}(X, M, N)$, then the measure space constructed in Remark 4.32 is $\sigma$-finite in the usual sense.

Definition 4.40. A (complex infinite) measure $\mu: M^{\prime} \rightarrow \mathbf{C}$ on an enhanced measurable space $(X, M, N)$ is essential if for any $m \in M$ such that $\left.\mu\right|_{m} \neq 0$ we can find a $\sigma$-finite $m^{\prime} \in M$ such that $m^{\prime} \subset m$ and $\left.\mu\right|_{m^{\prime}} \neq 0$.

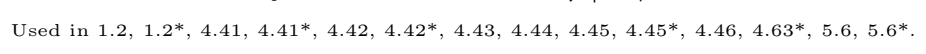

Proposition 4.41. Any essential (complex infinite) measure $\mu: M^{\prime} \rightarrow \mathbf{C}$ on an enhanced measurable space $(X, M, N)$ is completely additive: for any family $\left\{m_{i}\right\}_{i \in I}$ (countable or not) of elements of $M^{\prime}$ disjoint up to a negligible set (meaning $m_{i} \cap m_{j} \in N$ whenever $i \neq j$ ) that admits an essential supremum $S \in M$ and such that the $\operatorname{sum} \sum_{i \in I} \mu\left(m_{i}^{\prime}\right)$ converges absolutely for any family $\left\{m_{i}^{\prime}\right\}_{i \in I}$ satisfying $m_{i}^{\prime} \subset m_{i}$ and $m_{i}^{\prime} \in M^{\prime}$, we have

$$
S \in M^{\prime}, \quad \mu(S)=\sum_{i \in I} \mu\left(m_{i}\right) .
$$

Conversely, if $(X, M, N)$ is localizable, any completely additive measure is essential. Used in 1.2 .2 .19$.

Proof. Suppose $\mu$ is an essential measure and $\left\{m_{i}\right\}_{i \in I}$ is a family with indicated properties. The absolute convergence of $\sum_{i \in I} \mu\left(m_{i}^{\prime}\right)$ implies that the set $J=\left\{i \in I|\mu|_{m_{i}} \neq 0\right\}$ is countable. Set $S^{\prime}=\bigcup_{j \in J} m_{j} \in M$. By assumption, we have $S^{\prime} \in M^{\prime}$ and $\mu\left(S^{\prime}\right)=\sum_{i \in I} \mu\left(m_{i}\right)$. It remains to show that $S \backslash S^{\prime} \in M^{\prime}$ and $\mu\left(S \backslash S^{\prime}\right)=0$. Indeed, $S \backslash S^{\prime}$ is the essential supremum of $\left\{m_{i} \cap\left(S \backslash S^{\prime}\right)\right\}_{i \in I \backslash J}$ and the restriction of $\mu$ to any of these sets vanishes. Hence $\mu$ vanishes on all $\sigma$-finite elements of $M$, therefore $\left.\mu\right|_{S \backslash S^{\prime}}=0$ because $\mu$ is essential.

Conversely, suppose $\mu$ is completely additive. If $\mu$ is not essential, then there is $m \in M$ such that $\left.\mu\right|_{m^{\prime}}=0$ for any $\sigma$-finite $m^{\prime} \in M$ such that $m^{\prime} \subset m$. Since the induced enhanced measurable space $\left(m, M_{m}, N_{m}\right)$ is localizable, we can find a family $\left\{m_{i}\right\}_{i \in I}$ of $\sigma$-finite elements of $M_{m}$ disjoint up to a negligible set whose essential supremum equals $m$. By completely additivity of $\mu$ we must have $\mu(m)=0$, a contradiction. I

Proposition 4.42. Any essential (complex infinite) measure $\mu: M^{\prime} \rightarrow \mathbf{C}$ on an enhanced measurable space $(X, M, N)$ preserves essential suprema: for any directed subset $T \subset M^{\prime}$ that is closed under passage to measurable subsets and admits an essential supremum $S \in M^{\prime}\left(\right.$ Remark 4.35), the limit $\lim _{t \in T} \mu(t)$ exists and $\mu(S)=\lim _{t \in T} \mu(t)$. Conversely, if $(X, M, N)$ is localizable, then any measure that preserves essential suprema is essential. Used in $4.45^{*}$

Proof. Suppose $\mu$ is an essential measure and $T$ is a directed subset of $M^{\prime}$ that is closed under passage to measurable subsets and admits an essential supremum $S \in M^{\prime}$ (Remark 4.35). We want to show that the limit $\lim _{t \in T} \mu(t)$ exists and $\mu(S)=\lim _{t \in T} \mu(t)$. Replacing $(X, M, N)$ by $\left(S, M_{S}, N_{S}\right)$, we can assume $\mu$ to be finite, i.e., $M^{\prime}=M$, and $X=S$ is now the essential supremum of $T$. By linearity, we can assume $\mu$ to be real. By the classical Hahn-Jordan decomposition (Fremlin [2001, Theorem 231E]), we can further assume $\mu$ to be positive and finite. In particular, $\lim _{t \in T} \mu(t)=\sup _{t \in T} \mu(t)$ exists and $\sup _{t \in T} \mu(t) \leq \mu(S)$.

For any $n>0$, set $A_{n}$ to an element of $T$ such that $\sup _{t \in T} \mu(t)-\mu\left(A_{n}\right)<1 / n$ and $A_{n} \supset A_{n-1}$ if $n>1$. Denote by $F$ the union $\bigcup_{n>0} A_{n}$. By construction, $F \in M$ and for any $t \in T$ we have $\mu(t \backslash F)<1 / n$ for 
all $n>0$, so $\mu(t \backslash F)=0$. Furthermore, $S \backslash F$ is the essential supremum of $t \backslash F$ for all $t \in T$. Replacing $(X, M, N)$ by $\left(S \backslash F, M_{S \backslash F}, N_{S \backslash F}\right)$, we may now assume $\mu(t)=0$ for all $t \in T$, and we have to show that

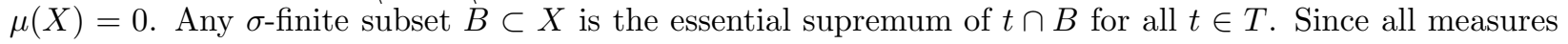
on $\sigma$-finite enhanced measurable spaces are essential, this implies that $\mu(B)=0$. Thus, $\mu(X)=0$ because $\mu$ is essential.

Conversely, suppose $\mu$ is a measure that preserves essential suprema. To show that $\mu$ is essential, suppose $m \in M$ is such that $\left.\mu\right|_{m^{\prime}}=0$ for all $\sigma$-finite $m^{\prime} \in M$ such that $m^{\prime} \subset m$. Since $(X, M, N)$ is localizable, $m$ is the essential supremum of all such $m^{\prime}$. This essential supremum is preserved by $\mu$, therefore, $\mu(m)=0$. I

Remark 4.43. If $(X, M, N)$ is an enhanced measurable space with a faithful semifinite measure $\mu$, then a map $\nu: M \rightarrow \mathbf{R}$ is a (finite real) measure on $(X, M, N)$ if and only if $\nu$ is countably additive and absolutely continuous with respect to $\mu$. Likewise, a map $\nu: M \rightarrow \mathbf{R}$ is an essential measure on $(X, M, N)$ if and only if $\nu$ is countably additive and truly continuous (Fremlin [2001, 232A(b)]) with respect to $\mu$. Thus, essential measures are precisely those measures that admit a Radon-Nikodym derivative with respect to $\mu$ (Fremlin 2001, 232E)). Used in 1.2.

Remark 4.44. Countably additive measures on localizable enhanced measurable spaces that are not essentia] exist if and only if real-valued-measurable cardinals exist (Fremlin [2002.d, Theorem 363S]). Indeed, if $X$ is such a cardinal with a probability measure $2^{X} \rightarrow[0,1]$ that vanishes on all countable subsets of $X$, then $2^{X}$ is a localizable Boolean algebra such that $\mu$ induces a probability measure $\nu$ on $\operatorname{Spec}\left(2^{X}\right)$ that vanishes on all rare subsets but does not preserve the essential supremum $X$ of the family of singleton subsets $\left\{\{x\} \in 2^{X} \mid x \in X\right\}$, since $\nu(\{x\})=0$, but $\nu(X)=1$. In particular, $\nu$ is not a normal measure because it is not a Radon measure due to its violation of the $\tau$-smoothness property. Used in $1.2^{*}$

Proposition 4.45. Given a localizable enhanced measurable space $(X, M, N)$, composition with the quotient map $M \rightarrow M / N$ establishes a canonical bijective correspondence from continuous valuations on $M / N$ to (finite) essential measures on $(X, M, N)$. Used in $1.2 *$, 4.37 , 4.46 . 5.6.

Proof. The quotient map $M \rightarrow M / N$ sends countable unions to countable joins. A valuation on $M / N$ restricts to a finitely additive measure on $M$ that vanishes on $N$, and this correspondence is bijective. A valuation is continuous if it sends suprema of directed subsets of $M / N$ to limits in $\mathbf{C}$, which translates into mapping essential suprema of directed subsets of $M$ to limits in C. By Proposition 4.42, the latter measures are precisely the essential measures. I

Remark 4.46. By definition, any measure $\mu$ on a 6 -finite enhanced measurable space $(X, M, N)$ is essential, so induces a continuous valuation on $M / N$ by Proposition 4.45. Used in 4.54*.

\subsection{Strictly localizable enhanced measurable spaces}

The problems described in Remark 5.18 justify our consideration of strictly localizable enhanced measurable spaces below.

Definition 4.48. An enhanced measurable space $(X, M, N)$ is strictly localizable (alias decomposable) if it is isomorphic in the category PreEMS to the coproduct of a small family of $\sigma$-finite enhanced measurable spaces. A specific choice of such a family will be referred to as a strictly localizing partition. The category of strictly localizable enhanced measurable spaces SLEMS is the full subcategory of EMS consisting of strictly

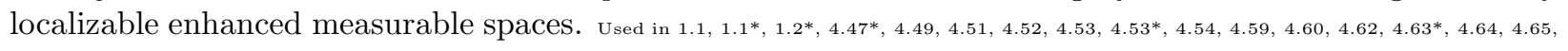
$5.65,5.4^{*}, 5.11^{*}, 5.12,5.15^{*}, 5.16^{*}, 5.18$

Example 4.49. (See Fremlin 2001, 216D].) Strictly localizable enhanced measurable spaces are not closed under isomorphisms in the category EMS. Pick an uncountable set $I$ and consider the enhanced measurable space $(X, M, N)$, where $X=\{0,1\} \times I, N$ is the set of countable subsets of $\{1\} \times I$, and $M$ consists of subsets of the form $(\{0,1\} \times J) \oplus n$, where $J \subset I$ and $n \in N$. The projection map $p:\{0,1\} \times I \rightarrow I$ is an isomorphism of enhanced measurable spaces $(X, M, N) \rightarrow\left(I, 2^{I},\{\emptyset\}\right)$ in the category EMS. The inverse map injects $I \cong\{0\} \times I$ into $\{0,1\} \times I$. The codomain $\left(I, 2^{I},\{\emptyset\}\right)$ is strictly localizable, but the domain $(X, M, N)$ is not. Indeed, elements of any strictly localizing partition of $(X, M, N)$ must be countable subsets of $X$. But then the set $\{0\} \times I$ has a measurable intersection with any element of such a partition, and yet $\{0\} \times I \notin M$ because $I$ is uncountable. Used in $1.1,4.49,4.62$.

The following definition will eliminate Example 4.49. 
Definition 4.50. The category LDEMS of locally determined enhanced measurable spaces is the full subcategory of EMS consisting of enhanced measurable spaces $(X, M, N)$ such for any $A \subset X$, if $A \cap F \in M$ (respectively $A \cap F \in N)$ for all $\sigma$-finite $F \in M$, then also $A \in M$ (respectively $A \in N$ ). Used in $1.1 .4 .4 .54,4.53$ $4.53 .4 .53 .4 .63,4.63 * 4.64 .5 .18$

Remark 4.51. We have an inclusion of full subcategories SLEMS $\subset$ LDEMS by Fremlin [2001, Theorem $211 \mathrm{~L}(\mathrm{~d})]$. Used in 4.52 .

Remark 4.52. Consider an enhanced measurable space $(X, M, N)$. Adding to $M$ respectively $N$ the subsets of $X$ that Definition 4.50 says must be elements of $M$ respectively $N$ produces a locally determined enhanced measurable space $\left(X, M^{\prime}, N^{\prime}\right)$ with $M^{\prime} \supset M$ and $N^{\prime} \supset N$ (Fremlin [2001, Proposition 213D]). Thus, the map $\left(X, M^{\prime}, N^{\prime}\right) \rightarrow(X, M, N)$ induced by the identity map on $X$ is a morphism of enhanced measurable spaces. If $(X, M, N)$ is localizable, then the induced homomorphism of Boolean algebras $M / N \rightarrow M^{\prime} / N^{\prime}$ is an isomorphism (Fremlin [2001, Proposition $213 \mathrm{H}(\mathrm{b})]$ ). However, the identity map on $X$ does not induce a morphism of enhanced measurable spaces $(X, M, N) \rightarrow\left(X, M^{\prime}, N^{\prime}\right)$ since $N^{\prime}$ can be bigger than $N$. Arguably, we want the identity map to be an isomorphism nevertheless. This can be achieved by modifying the definition of a premap of enhanced measurable spaces (Definition 4.6), replacing the condition $f^{*} n \in N_{X}$ for all $n \in N_{Y}$ with the condition that $f^{*} n \cap m \in N_{X}$ for all $n \in N_{Y}$ and $f$-finite $m \in M_{X}$. We did not adopt this modification for the same reason that we required completeness in the definition of enhanced measurable spaces: the resulting definition of a morphism of enhanced measurable spaces is the simplest possible. (Compare Definition 4.4 with Remark 4.8 for the noncomplete case.) Since we quickly restrict to strictly localizable enhanced measurable spaces, which are ocally determined by Remark 4.51, the difference is irrelevant for us anyway. The category LDEMS is only used in purely expository Lemma 4.53, Lemma 4.63, and Remark 5.18, which are not used in the main results.

Lemma 4.53. Strictly localizable enhanced measurable spaces are closed under isomorphisms in the category LDEMS. Used in $4.52,4.63$.

Proof. Suppose $f:\left(X, M_{X}, N_{X}\right) \rightarrow\left(Y, M_{Y}, N_{Y}\right)$ is an isomorphism in LDEMS with a trictly localizable codomain. Fix a strictly localizing partition $\left\{B_{i}\right\}_{i \in I}$ for $\left(Y, M_{Y}, N_{Y}\right)$ together with a finite measure $\mu_{i}$ (for each $i \in I)$ on $\left(Y, M_{Y}, N_{Y}\right)$ such that for any $m \in M_{Y}$ we have $\mu_{i}(m)=0$ if and only if $m \cap B_{i} \in N_{Y}$. The sets $A_{i}=f^{*} B_{i}$ and (if nonempty) $X \backslash$ pdom $f$ form a partition of $X$ into nonempty measurable subsets of $X$. For any $i \in I$ the pushforward measure $\nu_{i}:=\left(f^{-1}\right)_{*}\left(\mu_{i}\right)$ satisfies the following condition: for any $m \in M_{X}$ we have $\nu_{i}(m)=0$ if and only if $m \cap A_{i} \in N_{X}$. We claim that the partition $\left\{A_{i}\right\}_{i \in I} \cup\{X \backslash$ pdom $f\}$ is a strictly localizing partition for $\left(X, M_{X}, N_{X}\right)$. This follows from Fremlin 2001, Proposition 213O(a)]. I

Proposition 4.54. Strictly localizable enhanced measurable spaces are localizable, used in 1.11 .

Proof. The Boolean algebra $M / N$ associated to a coproduct of enhanced measurable spaces in the category PreEMS is the product of the Boolean algebras associated to individual members of the family. Since the inclusion LBAlg $\rightarrow$ BAlg preserves products, it suffices to show that $\sigma$-finite enhanced measurable spaces are localizable. By definition of a $\sigma$-finite enhanced measurable space, it admits a faithful finite measure $\mu$, which descends to a faithful finite continuous valuation $\nu$ on $M / N$ by Remark 4.46. The Boolean algebra $M / N$ admits suprema of countable subsets because the Boolean algebra $M$ does and the ideal $N \subset M$ is closed under suprema of countable subsets. By Lemma 4.55, the Boolean algebra $M / N$ is complete. Thus, $M / N$ belongs to LBAlg. I

Lemma 4.55. Suppose a Boolean algebra $A$ admits a faithful continuous valuation $\nu: A \rightarrow[0, \infty)$. If $A$ admits suprema of countable subsets, then $A$ is complete. In addition, if $A^{\prime}$ is a complete Boolean algebra and $f: A \rightarrow A^{\prime}$ is a homomorphism of Boolean algebras that preserves countable suprema, then $f$ is complete. Used in $4.54,4.654,0.11$.

Proof. Suppose $S \subset A$ is an arbitrary subset of $A$, which we may assume to be closed under finite joins, which does not alter suprema. We want to show that $\sup S$ exists. It suffices to construct a countable subset $T \subset S$ such that for any $s \in S$ we have $s \leq \sup T$, which implies that $\sup S=\sup T$ exists.

Set $r=\sup _{s \in S} \nu(s)$, which exists because $\nu(s) \leq \nu(1)$. Choose a countable subset $T \subset S$ (which we again may assume to be closed under finite joins) such that $r=\sup _{t \in T} \nu(t)$ and set $\tau=\sup T$, so that $\nu(\tau) \geq r$. 
For any $s \in S$ we have

$$
\nu(s \vee \tau)=\nu(s \vee \sup T)=\nu\left(\sup _{t \in T}(s \vee t)\right) \leq \nu(\sup S) \leq r
$$

because $s \vee t \in S$ and $\nu(\sup S)=\sup _{s \in S} \nu(s)=r$ by continuity of $\nu$. Thus,

$$
\nu(s \backslash \tau)=\nu((s \vee \tau) \backslash \tau)=\nu(s \vee \tau)-\nu(\tau) \leq r-\nu(\tau) \leq 0,
$$

so $\nu(s \backslash \tau)=0$ and $s \backslash \tau=0$ because $\nu$ is faithful. Hence, $s \leq \tau=\sup T$ as desired.

To show that the map $f$ preserves arbitrary suprema, consider an arbitrary subset $S \subset A$, which we may assume to be directed. Using the above construction, choose a countable directed subset $T \subset S$ such that $\sup S=\sup T$. Now $f(\sup S)=f(\sup T)=\sup f_{!} T \leq \sup f_{!} S$. Also, $\sup f_{!} S \leq f(\sup S)$ follows from $f(s) \leq f(\sup S)$ for any $s \in S$. Hence $f(\sup S)=\sup f ! S$, i.e., $f$ preserves arbitrary suprema. I

\subsection{Compact enhanced measurable spaces}

The following definition was introduced (with an additional countability condition) by Edward Marczewski [1953, §4]. An extensive treatment of the underlying theory was given by Pfanzagl-Pierlo [1966].

Definition 4.57. A collection $K \subset 2^{X}$ of subsets of a set $X$ is a compact class if for any $K^{\prime} \subset K$ the following finite intersection property holds: if for any finite $K^{\prime \prime} \subset K^{\prime}$ we have $\bigcap K^{\prime \prime} \neq \emptyset$, then also $\bigcap K^{\prime} \neq \emptyset$. A compact enhanced measurable space is an enhanced measurable space $(X, M, N)$ for which there is a compact class $K \subset M$ such that for any $m \in M \backslash N$ there is $k \in K \backslash N$ such that $k \subset m$. Used in 1.1 .1 .1 .4 .2 .4 .

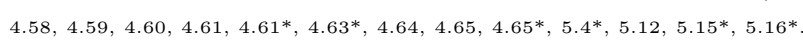

Remark 4.58. The choice of terminology is motivated by the fact (Fremlin [2002.d, Lemma 342D(a)]) that $K \subset 2^{X}$ is a compact class if and only if there is a compact topology on the set $X$ such that every element of $K$ is a closed (and hence compact) subset of $X$. Thus, an enhanced measurable space is compact if and only if it admits a compact topology such that every nonnegligible measurable set contains a nonnegligible subset that is closed (Fremlin 2002.d, Corollary 342F]).

Definition 4.59. The category of compact strictly localizable enhanced measurable spaces CSLEMS is the full subcategory of EMS consisting of enhanced measurable spaces that are compact (Definition 4.57) and

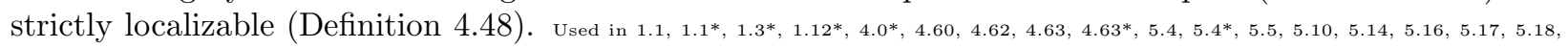
5.19 .

Example 4.60. The class of compact strictly localizable enhanced measurable spaces contains Radon enhanced measurable spaces, i.e., enhanced measurable spaces equipped with a structure of a Hausdorff topological space such that the $\sigma$-algebra of measurable sets contains open sets and there is a faithful measure $\mu$ that is locally finite (every point has a neighborhood of finite measure) and inner regular with respect to compact subsets (the measure of any measurable subset is the supremum of measures of its compact subsets). (See Fremlin 2003, Definition 411H(b)].) Thus, CSLEMS includes the vast majority of enhanced measurable spaces used in analysis.

Indeed, any Radon enhanced measurable space is strictly localizable by Fremlin [2003, Theorem 414J]. Also, compact subsets of a Hausdorff topological space form a compact clas because compact subsets of Hausdorff spaces are closed. Furthermore, the inner regularity property implies that for any $m \in M \backslash N$ we have $\mu(m)=\sup _{k \subset m} \mu(k)>0$, so there is $k \subset m$ such that $k$ is compact and $k \in K \backslash N$, as required by the definition of a compact enhanced measurable space. Slightly more generally, compactness continues to hold for non-Hausdorff Radon enhanced measurable spaces, which are defined using closed compact subsets instead of compact subsets. Used in 1.1 .

Lemma 4.61. If $(X, M, N)$ is a compact enhanced measurable space and $m \in M$, then the nduced enhanced measurable space $\left(m, M_{m}, N_{m}\right)$ is also compact. Used in t.11.

Proof. If $K \subset M$ is a compact class that exhibits the compactness of $(X, M, N)$, then $K \cap M_{m}$ is a compact class that exhibits the compactness of $\left(m, M_{m}, N_{m}\right)$. I 
Example 4.62. Compact strictly localizable enhanced measurable spaces are not closed under isomorphisms in the category EMS. Indeed, Example 4.49 constructs an isomorphism $p$ of enhanced measurable spaces whose domain is not strictly localizable and codomain is strictly localizable. Both domain and codomain are compact, as witnessed by the compact class $\{\{0\} \times\{i\}\}_{i \in I}$ for the domain and $\{\{i\}\}_{i \in I}$ for the codomain. Used in $1.1^{+}$.

The proof of the following lemma resembles Part $(\gamma)$ of the proof of Theorem in $\S 3$ of Fremlin 1999] or Part (g) of the proof of Theorem 343B in Fremlin [2002.d].

Lemma 4.63. Compact strictly localizable enhanced measurable spaces are closed under isomorphisms in the category LDEMS (Definition 4.50). Used in 6.1 .4 .4 .52 . 5.18

Proof. Suppose $f:\left(X, M_{X}, N_{X}\right) \rightarrow\left(Y, M_{Y}, N_{Y}\right)$ is an isomorphism in EMS whose codomain is a compact strictly localizable enhanced measurable space. By Lemma 4.53, the enhanced measurable spaces $\left(X, M_{X}, N_{X}\right)$ is strictly localizable because it is locally determined. If each element of a strictly localizing partition of $(X, M, N)$ is compact, then the union of compact classes of each part is a compact class for $\left(X, M_{X}, N_{X}\right)$. Thus we may assume $\left(Y, M_{Y}, N_{Y}\right)$ to be $\sigma$-finite.

Denote by $g=f^{-1}:\left(Y, M_{Y}, N_{Y}\right) \rightarrow\left(X, M_{X}, N_{X}\right)$ the inverse of $f$ in the category EMS. Observe that $M_{X}=N_{X}$ if and only if $M_{Y}=N_{Y}$, in which case $\left(X, M_{X}, N_{X}\right)$ is compact because $M_{X} \backslash N_{X}=\emptyset$. Otherwise, we can always choose point-set representatives for $f$ and $g$ so that their point-set domains have empty complements, i.e., both $f$ and $g$ are everywhere defined. Observe that $f^{*}\left(M_{Y} \backslash N_{Y}\right) \subset M_{X} \backslash N_{X}$ and $g^{*}\left(M_{X} \backslash N_{X}\right) \subset M_{Y} \backslash N_{Y}$.

Suppose $K_{Y} \subset M_{Y}$ is a compact clas that exhibits the compactness of $Y$. If $K_{Y}^{\prime} \supset K_{Y}$ is a bigger compact class, then $K_{Y}^{\prime}$ also exhibits the compactness of $Y$. In particular, we may assume $K_{Y}$ to be closed under finite unions and countable intersections. Take $K_{X}$ to be the set of $k_{X} \in M_{X}$ such that there is $k_{Y} \in K_{Y}$ for which $g_{!} k_{Y} \subset k_{X} \subset f^{*} k_{Y}$. We fix such $k_{Y}$ for each $k_{X} \in K_{X}$ and denote it by $L_{k_{X}}$. We claim that the set $K_{X}$ is a compact class that exhibits the compactness of $\left(X, M_{X}, N_{X}\right)$.

To show that $K_{X}$ is a compact class, suppose that $K^{\prime} \subset K_{X}$ has the finite intersection property, i.e., for any finite $K^{\prime \prime} \subset K^{\prime}$ we have $\bigcap K^{\prime \prime} \neq \emptyset$. We have $f^{*}\left(\bigcap_{k \in K^{\prime \prime}} L_{k}\right)=\bigcap_{k \in K^{\prime \prime}} f^{*}\left(L_{k}\right) \supset \bigcap_{k \in K^{\prime \prime}} k \neq \emptyset$, so $\bigcap_{k \in K^{\prime \prime}} L_{k} \neq \emptyset$. Thus, the family $\left\{L_{k}\right\}_{k \in K^{\prime}}$ has the finite intersection property and $W=\bigcap_{k \in K^{\prime}} L_{k} \neq \emptyset$. Now $g_{!} W \subset g_{!}\left(L_{k}\right) \subset k$ for all $k \in K^{\prime}$, so $g ! W \subset \bigcap_{k \in K^{\prime}} k$, and hence $\bigcap_{k \in K^{\prime}} k \neq \emptyset$, i.e., $K_{X}$ is a compact class.

We now show that $K_{X}$ exhibits the compactness of $X$. Fix a faithful finite measure $\mu$ on $\left(X, M_{X}, N_{X}\right)$ and its pushforward $\nu=f_{*} \mu$ on $\left(Y, M_{Y}, N_{Y}\right)$. Given $m \in M_{X} \backslash N_{X}$, we must demonstrate that there is $k_{X} \in K_{X} \backslash N_{X}$ such that $k_{X} \subset m$. To this end, we also construct $k_{Y} \in K_{Y} \backslash N_{Y}$ such that $g_{!} k_{Y} \subset k_{X} \subset f^{*} k_{Y}$. Fix some $\gamma>0$ such that $\gamma<\mu(m)$.

Construct sequences $\left\{V_{n}\right\}_{n \geq 0}$, where $V_{n} \in M_{Y} \backslash N_{Y}, \nu\left(V_{n}\right)>\gamma$, and $\left\{F_{n}\right\}_{n \geq 0}$, where $F_{n} \in M_{X} \backslash N_{X}$, $\mu\left(F_{n}\right)>\gamma$ by induction as follows. Set $F_{0}=m$. Suppose we already constructed $F_{k}$ for $k \leq n$ and $V_{k}$ for $k<n$. Observe that $g^{*} F_{n} \in M_{Y} \backslash N_{Y}$ because $\nu\left(g^{*} F_{n}\right)=\mu\left(F_{n}\right)>\gamma$. Consider the set $Q$ of all $R \in K_{Y} \backslash N_{Y}$ such that $R \subset g^{*} F_{n}$ and $\nu(R)>0$, which is nonempty by compactness of $\left(Y, M_{Y}, N_{Y}\right)$ exhibited by $K_{Y}$ and is closed under finite unions by definition of $K_{Y}$. Denote by $P$ the essential supremum of $Q$. We have $\nu(P)=\nu\left(g^{*} F_{n}\right)$, since otherwise $g^{*} F_{n} \backslash P \in M_{Y} \backslash B_{Y}$ would contain some $R \in K_{Y} \backslash N_{Y}$ such that $R \subset g^{*} F_{n} \backslash P$ and $\nu(R)>0$, and hence $R \in Q$, contradicting the definition of $P$. Now set $V_{n}$ to an element $R \in Q$ such that $\nu(R)>\gamma$. Such an element exists because $\nu(P)=\nu\left(g^{*} F_{n}\right)=\mu\left(F_{n}\right)>\gamma$ and $\nu$ is an essential measure.

Set $F_{n+1}$ to $f^{*} V_{n} \backslash\left(f^{*} g^{*} F_{n} \backslash F_{n}\right)$. Observe that $f^{*} g^{*} F_{n} \backslash F_{n} \in N_{X}$, so $\mu\left(F_{n+1}\right)=\mu\left(f^{*} V_{n}\right)=\nu\left(V_{n}\right)>\gamma$. Also $F_{n+1} \subset F_{n}$ by construction.

After the induction, set $k_{X}=\bigcap_{n \geq 0} F_{n}$ and $k_{Y}=\bigcap_{n \geq 0} V_{n}$. We have $k_{Y} \in K_{Y}$ because $V_{n} \in K_{Y}$ by construction and $K_{Y}$ is closed under countable intersections. We have

$$
k_{Y}=\bigcap_{n \geq 0} V_{n} \subset \bigcap_{n \geq 0} g^{*} F_{n}=g^{*} \bigcap_{n \geq 0} F_{n}=g^{*} k_{X},
$$

so $g ! k_{Y} \subset k_{X}$. Also

$$
k_{X}=\bigcap_{n \geq 0} F_{n} \subset \bigcap_{n \geq 0} F_{n+1} \subset \bigcap_{n \geq 0} f^{*} V_{n}=f^{*} \bigcap_{n \geq 0} V_{n}=f^{*} k_{Y} .
$$


Altogether,

$$
g ! k_{Y} \subset k_{X} \subset f^{*} k_{Y},
$$

which shows that $k_{X} \in K_{X}$. Furthermore, $\mu\left(k_{X}\right)=\inf _{n \geq 0} \mu\left(F_{n}\right) \geq \gamma>0$, so $k_{X} \in K_{X} \backslash N_{X}$. By construction, $k_{X} \subset F_{0}=m$, which completes the proof. I

Remark 4.64. The argument in the last paragraph of the above proof heavily uses the existence of a faithful finite measure obtained by exploiting strict localizability. Although it is not necessary below, it would be interesting to know whether compact enhanced measurable spaces are closed under isomorphisms in LDEMS, and if not, whether one can impose a condition weaker than strict localizability that would guarantee such closedness.

Proposition 4.65. Suppose $(X, M, N)$ is a compact enhanced measurable space that admits a faithfu semifinite measure, $\left(X^{\prime}, M^{\prime}, N^{\prime}\right)$ is a strictly localizable enhanced measurable space, and $f:(X, M, N) \rightarrow$ $\left(X^{\prime}, M^{\prime}, N^{\prime}\right)$ is a morphism of enhanced measurable spaces. Then $f$ has a well-defined measurable image $m \in M^{\prime}$ in the following sense: $f^{*}(X \backslash m) \in N$ and for any $m^{\prime} \in M^{\prime}$ such that $m^{\prime} \subset m$ and $f^{*} m^{\prime} \in N$ we have $m^{\prime} \in N^{\prime}$. The class of $m$ in $M^{\prime} / N^{\prime}$ is unique. Used in $6.11^{*} 5.11^{*}$.

Proof. Uniqueness is easy to show: if $m_{0}$ and $m_{1}$ are two elements with such properties, then $f^{*}\left(m_{0} \backslash m_{1}\right) \subset$ $f^{*}\left(X \backslash m_{1}\right) \in N$, so $m_{0} \backslash m_{1} \in N^{\prime}$. Likewise, $m_{1} \backslash m_{0} \in N^{\prime}$ and hence $m_{0} \oplus m_{1} \in N^{\prime}$, so $m_{0}$ and $m_{1}$ map to the same element in $M^{\prime} / N^{\prime}$. To show existence, denote by $p \in M^{\prime}$ the essential supremum of all $m^{\prime} \in M^{\prime}$ such that $f^{*} m^{\prime} \in N$. We claim that the element $m=X \backslash p \in M^{\prime}$ has the desired properties. First, if $m^{\prime} \subset m$ and $f^{*} m^{\prime} \in N$, then $m^{\prime} \backslash p \in N^{\prime}$. Since $m^{\prime} \backslash p=m^{\prime}$ because $m^{\prime} \subset m$ and $m \cap p=\emptyset$, we have $m^{\prime} \in N^{\prime}$.

Secondly, to show that $f^{*} p \in N$, choose a strictly localizing partition $\left\{q_{i}\right\}_{i \in I}$ for $\left(X^{\prime}, M^{\prime}, N^{\prime}\right)$. Set $r_{i}=q_{i} \cap p$. We claim that $f^{*} r_{i} \in N$. Indeed, $r_{i}$ is the essential supremum of the collection $R_{i}=\{m \in$ $\left.M^{\prime} \mid m \subset q_{i}, f^{*} m \in N\right\}$. The Induced enhanced measurable space of $q_{i}$ is $\sigma$-finite, so Lemma 4.55 supplies a countable directed subset $R_{i}^{\prime} \subset R_{i}$ with the same essential supremum. Since $R_{i}^{\prime}$ is countable, its essential supremum can be computed as the union. Since $f^{*}$ preserves unions and $N$ is a $\sigma$-ideal, we deduce that $f^{*} r_{i} \in N$.

Thus, the induced enhanced measurable space of $f^{*} p$ is a compact enhanced measurable space that admits a faithful semifinite measure $\mu$ and is partitioned into a disjoint family of negligible subsets $\left\{f^{*} r_{i}\right\}_{i \in I}$. Furthermore, by definition of a strictly localizing partition, for any subset $J \subset I$ we have $\bigcup_{i \in J} f^{*} r_{i} \in M$. By Fremlin [2003, Lemma 451Q], we have $\bigcup_{i \in I} f^{*} r_{i}=f^{*} p \in N$, which completes the proof. I 


\section{$5 \quad$ Equivalence between measurable spaces and measurable locales}

\subsection{From hyperstonean spaces to enhanced measurable spaces used in 5.7, 5.4.5.4.}

Recall that a continuous map of topological spaces is open if images of open subsets are open. The category of topological spaces and open maps is denoted by TopOpen.

The construction of an enhanced measurable space out of a topological space given below was already known to Loomis [1947] and Sikorski [1948], who defined the enhanced measurable space denoted below by $\operatorname{Spec}(A)$ for an arbitrary $\sigma$-complete Boolean algebra $A$ by applying Definition 5.2 to the Stone space of $A$, and proved that its Boolean algebra of equivalences classes of measurable sets modulo negligible sets is isomorphic to $A$.

Definition 5.2. We define a functor

$$
\text { TM: TopOpen } \rightarrow \text { EMS }
$$

(TM for topology-to-measure) by sending a topological space $(X, U)$ to the enhanced measurable space $\mathbb{T M}(X, U)=(X, M, N)$ defined as follows. Define $N$ to be the collection of all meager subsets of $X$. Define $M$ to be the collection of all subsets of $X$ with the property of Baire, i.e., symmetric differences of elements of $U$ and $N$. An open continuous map $f:(X, U) \rightarrow\left(X^{\prime}, U^{\prime}\right)$ of topological spaces is sent to the morphism

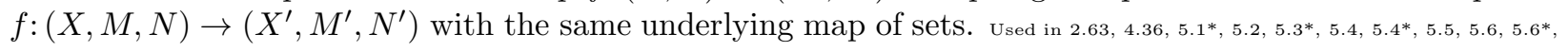
. 15.

Lemma 5.3. This definition is correct. Used in 1.04

Proof. Lemma 2.68 proves that $M$ is a $\sigma$-algebra and $N \subset M$ is a $\sigma$-idea of subsets of $X$. Thus, $(X, M, N)$ is an enhanced measurable space.

Now suppose $f: S \rightarrow S^{\prime}$ is an open map and $\mathrm{TM}(f):(X, M, N) \rightarrow\left(X^{\prime}, M^{\prime}, N^{\prime}\right)$ is the induced map of enhanced measurable spaces. We have to show that $\mathrm{TM}(f)^{*}$ sends elements of $M^{\prime}$ to $M$ and $N^{\prime}$ to $N$. Since $f$ is continuous and $f^{*}$ preserves symmetric differences and countable unions, it suffices to show that $f^{*}$ preserves closed subsets with empty interiors. Indeed, if $V \subset X^{\prime}$ is a closed subset with an empty interior and $U \subset f^{*} V$ is an open subset, then $f(U) \subset V$ is also an open subset because $f$ is an open map, and hence $f(U)=\emptyset$ and $U=\emptyset$, so $f^{*} V \subset X$ is a closed subset with an empty interior. I

Proposition 5.4. The functor

$$
\text { TM: TopOpen } \rightarrow \text { EMS }
$$

restricts to a functor

$$
\text { TM: HStonean } \rightarrow \text { CSLEMS. }
$$

Used in 1.1 .

Proof. Recall that HStonean is a full subcategory of TopOpen, so it suffices to show that TM sends a hyperstonean space $S$ to a compact strictly localizable enhanced measurable space. To show strict localizability, use Zorn's lemma to choose a maximal disjoint family $\left\{a_{i}\right\}_{i \in I}$ of nonzero elements of the complete Boolean algebra $A=$ COpen $(\Omega(S))$ such that the complete Boolean algebra $a_{i} A$ admits a faithful finite continuous valuation for any $i \in I$. In particular, every $a_{i} \subset S$ can be chosen to be a clopen subset of $S$, which admits a finite measure supported on it. The clopen subsets $a_{i} \subset S$ are disjoint and their union is an open subset $b \subset S$ such that $\neg \neg b=S$, so $S \backslash b$ is meager. The partition of $S$ into disjoint $\sigma$-finite measurable subsets $\left\{a_{i}\right\}_{i \in I}$ and $S \backslash b$ exhibits TM $(S)$ as a strictly localizable enhanced measurable space. Indeed, if $\left\{n_{i}\right\}_{i \in I}$ is a collection of negligible (i.e., rare) subsets of $a_{i}$, then $\bigcup_{i \in I} n_{i}$ is rard, hence negligible. Likewise, if $\left\{u_{i} \oplus n_{i}\right\}_{i \in I}$ is a collection of measurable subsets of $a_{i}$, then $\bigcup_{i \in I}\left(u_{i} \oplus n_{i}\right)=\bigcup_{i \in I} u_{i} \oplus \bigcup_{i \in I} n_{i}$ is measurable because $\bigcup_{i \in I} u_{i}$ is open and $\bigcup_{i \in I} n_{i}$ is rare.

To show compactness, recall that hyperstonean spaces are compact, so their clopen subsets are also compact. Thus the class of all clopen subsets of $S$ is a compact class. It remains to show that any nonnegligible measurable subset $a \subset \mathbb{T M}(S)$ contains a nonempty clopen subset. Since any open subset of TM $(S)$ is a union of clopen subsets, it suffices to show that $a$ contains a nonempty open subset. By definition of TM, the subset $a$ is the symmetric difference $u \oplus n$ of an open subset $u$ and a meager subset $n$. The subset $u$ is nonempty because $a$ is nonnegligible. It suffices to show that the subset $n$ is rare, since then $u \backslash \bar{n}$ is a 
nonempty open subset of $a$. By definition of a rare set, it suffices to show that $\bar{n}$ is rare. Denote by $\hat{n}$ the interior of $\bar{n}$. The difference $\bar{n} \backslash \hat{n}$ is rare, so for any hormal measure $\mu$ on $S$ we have $0=\mu(n)=\mu(\bar{n})=\mu(\hat{n})$, which by definition of a hyperstonean space implies $\hat{n}=\emptyset$, i.e., $n$ is rare. This shows that TM $(S)$ is a compact enhanced measurable space. I

Definition 5.5. We define a functor

$$
\text { Spec: MLod } \rightarrow \text { CSLEMS }
$$

as the composition

$$
\begin{aligned}
& \text { MLoo } \stackrel{\text { Ideal }}{\longrightarrow} \text { HStoneanLod } \stackrel{\mathrm{Sp}}{\longrightarrow} \text { HStonean } \stackrel{\mathrm{TM}}{\longrightarrow} \text { CSLEMS. }
\end{aligned}
$$

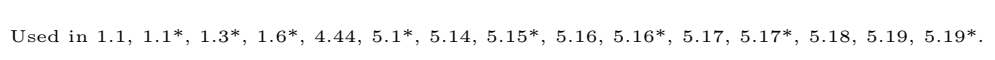

Proposition 5.6. For any hyperstonean space $S$ there is a canonical bijective correspondence between normal measures on $S$ and essential measures on TM $(S)=(S, M, N)$.

Proof. Combine Proposition 4.45, Remark 2.70, and Lemma 2.58. Alternatively, observe directly that any essential measure on $\mathrm{TM}(S)$ restricts to a hormal measure on $S$ (with the essentiality property implying $\tau$-smoothness, which is equivalent to being a Radon measure) and conversely, any normal measure on $S$ extends to a unique essential measure on $\mathrm{TM}(S)$ because it automatically vanishes on elements of $N$. I

\subsection{From enhanced measurable spaces to measurable locales}

Definition 5.8. The functor PreML: PreEMS $\rightarrow$ BAlg 0 sends an enhanced measurable space $(X, M, N)$ to the quotient Boolean algebra $M / N$ and a map of enhanced measurable spaces

$$
f:(X, M, N) \rightarrow\left(X^{\prime}, M^{\prime}, N^{\prime}\right)
$$

to the morphism

$$
\operatorname{PreML}(f): M / N \rightarrow M^{\prime} / N^{\prime}
$$

in BAlgop given by the map of Boolean algebras $M^{\prime} / N^{\prime} \rightarrow M / N$ induced by the map $f^{*}: M^{\prime} \rightarrow M$. Furthermore, the functor PreML descends to functors

$$
\text { StrictML: StrictEMS } \rightarrow \text { BAlg }
$$

and

$$
\text { WeakML: EMS } \rightarrow \text { BAlg }
$$

Used in 5.8 .5 .9 .5 .5 .10 .5 .11 .5 .18

Lemma 5.9. The above definition is correct.

Proof. By definition of a $\sigma$-algebra and $\sigma$-ideal, $N$ is an ideal of the Boolean algebra $M$, so the quotient $M / N$ is a Boolean algebra. The map $f^{*}: M^{\prime} \rightarrow M$ preserves all Boolean operations and it sends the $\sigma$-ideal $N^{\prime}$ to the $\sigma$-ideal $N$ by definition of an enhanced measurable space. Hence, $\operatorname{PreML}(f)^{*}: M^{\prime} / N^{\prime} \rightarrow M / N$ is a homomorphism of Boolean algebras. Composition is preserved because $(g \circ f)^{*}=f^{*} \circ g^{*}$. Likewise, identities are preserved, which proves that we indeed have a functor PreML

Premaps of enhanced measurable spaces that are weakly equal almost everywhere by definition induce identical morphisms $M^{\prime} / N^{\prime} \rightarrow M / N$. This shows that the functor

$$
\text { PreML:PreEMS } \rightarrow \text { BAlg }
$$

descends to a functor

$$
\text { WeakML:EMS } \rightarrow \text { BAlg }
$$

and hence also to a functor

$$
\text { StrictML: StrictEMS } \rightarrow \text { BAlgp, }
$$

since EMS is a quotient of StrictEMS. I 
Definition 5.10. The functor

$$
M L: \text { CSLEMS } \rightarrow \text { MLod. }
$$

(ML for measure-to-locale) is the restriction of the functor WeakML to the corresponding subcategories.

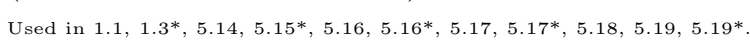

Lemma 5.11. The above definition is correct. Used in $5.13,5.12$

Proof. By Proposition 4.54, the functor WeakML sends strictly localizable enhanced measurable spaces to localizable Boolean algebras. It also sends a morphism $f:(X, M, N) \rightarrow\left(X^{\prime}, M^{\prime}, N^{\prime}\right)$ of strictly localizable enhanced measurable spaces to the homomorphism WeakML $(f)$ op : $M^{\prime} / N^{\prime} \rightarrow M / N$ of Boolean algebras induced by the homomorphism $f^{*}: M^{\prime} \rightarrow M$. The map $f^{*}$ preserves countable suprema, and hence so does WeakML $(f)$ op.

To show that the map WeakML $(f)$ ap also preserves arbitrary suprema, we reduce to the case of $\sigma$-finite enhanced measurable spaces. By Definition 4.48, we can represent $(X, M, N)$ as a coproduct $\coprod_{i \in I}\left(X_{i}, M_{i}, N_{i}\right)$ of $\sigma$-finite enhanced measurable spaces so that $M / N=\prod_{i \in I} M_{i} / N_{i}$. These spaces are compact by Lemma 4.61. The map

$$
\text { WeakML }(f) \text { op: } M^{\prime} / N^{\prime} \rightarrow M / N=\prod_{i \in I} M_{i} / N_{i}
$$

preserves arbitrary suprema if and only if its individual components $M^{\prime} / N^{\prime} \rightarrow M_{i} / N_{i}$ preserve suprema for all $i \in I$. Thus, we may assume that $(X, M, N)$ is a compact $\sigma$-finite enhanced measurable space.

By Proposition 4.65, we have an element $X_{1}^{\prime} \in M^{\prime}$ such that $f^{*}\left(X^{\prime} \backslash X_{1}^{\prime}\right) \in N$ and for any $m^{\prime} \in M^{\prime}$ such that $m^{\prime} \subset X_{1}^{\prime}$ and $f^{*} m^{\prime} \in N$ we have $m^{\prime} \in N^{\prime}$. The Boolean algebra $M^{\prime} / N^{\prime}$ splits as the product of Boolean algebras $M_{1}^{\prime} / N_{1}^{\prime}$ and $M_{2}^{\prime} / N_{2}^{\prime}$, where $\left(X_{1}^{\prime}, M_{1}^{\prime}, N_{1}^{\prime}\right)$ and $\left(X_{2}^{\prime}, M_{2}^{\prime}, N_{2}^{\prime}\right)$ are the induced enhanced measurable spaces of $X_{1}^{\prime}$ and $X_{2}^{\prime}=X^{\prime} \backslash X_{1}^{\prime}$. By construction, the homomorphism of Boolean algebras WeakML $(f)$ op is injective on $M_{1}^{\prime} / N_{1}^{\prime}$ and vanishes on $M_{2}^{\prime} / N_{2}^{\prime}$. Thus, we can replace $\left(X^{\prime}, M^{\prime}, N^{\prime}\right)$ with $\left(X_{1}^{\prime}, M_{1}^{\prime}, N_{1}^{\prime}\right)$ and assume WeakML $(f)$ or to be injective.

If $\mu$ is a faithful finite measure on $(X, M, N)$, then $f_{*} \mu=\mu \circ f^{*}$ is a faithful finite measure on $\left(X^{\prime}, M^{\prime}, N^{\prime}\right)$. Thus, the map WeakML $(f)$ op: $M^{\prime} / N^{\prime} \rightarrow M / N$ is an injective homomorphism of Boolean algebras that preserves countable suprema and its domain and codomain admit faithful (finite) continuous valuations. By Lemma 4.55 the map WeakML $(f)$ op preserves arbitrary suprema, which completes the proof. I

Remark 5.12. Consider an uncountable set $X$ with a measure $\mu: 2^{X} \rightarrow[0,1]$ that vanishes on all singletons. Such measures can be constructed in presence of sufficiently large cardinals, see Fremlin 1993]. Set $N$ to the $\sigma$-ideal of subsets of $X$ on which $\mu$ vanishes. The identity map $X \rightarrow X$ yields a morphism $\left(X, 2^{X}, N\right) \rightarrow$ $\left(X, 2^{X},\{\emptyset\}\right)$ in the category EMS. The domain is a 6 -finite enhanced measurable space and the codomain is a strictly localizable enhanced measurable space. The induced homomorphism of Boolean algebras $2^{X} \rightarrow$ $2^{X} / N$ does not preserve suprema because all singletons in $X$ map to 0 , but their supremum in $2^{X}$ is $X$, which maps to the nonzero class of $X$ in $2^{X} / N$. Thus, we really need compactness in the statement of Lemma 5.11. Used in $5.13 * 5.19$.

\subsection{Equivalence of compact strictly localizable enhanced measurable spaces and measurable locales}

Definition 5.14. The natural isomorphism $\varepsilon$ is defined as a natural transformation from the composition

$$
\mathrm{MLO} \stackrel{\mathrm{Spec}}{\longrightarrow} \mathrm{CSLEMS} \stackrel{\mathrm{ML}}{\longrightarrow} \mathrm{MLod}
$$

to the identity functor on MLod that sends a measurable locale $L$ to the isomorphism of locales

$$
\varepsilon_{L}: \operatorname{ML}(\operatorname{Spec}(L)) \rightarrow L
$$

whose associated map of frames $\varepsilon_{L}^{*}: L \rightarrow \mathrm{ML}(\operatorname{Spec}(L))$ sends $m \in L$ to the equivalence class of the clopen subset of $\operatorname{Spec}(L)$ corresponding to the open element of Ideal $(L)$ given by the principal ideal of $m$.

Lemma 5.15. This definition is correct.

Proof. Lemma 2.68 shows that any measurable subset of $\operatorname{Spec}(L)$ has a unique presentation as the symmetric difference of a clopen subset and a $\operatorname{rar}$ subset of $\operatorname{Spec}(L)$. Since negligible subsets of $\operatorname{Sped}(L)$ coincide 
with rare subsets, the Boolean algebra $\operatorname{ML}(\operatorname{Spec}(L))$ is canonically isomorphic to the Boolean algebra of clopen subsets of $\operatorname{Sped}(L)$, equivalently, clopen subsets of $\operatorname{Sp}(\operatorname{Idea}(L))$, i.e., COpen(Ideal $(L)) \cong L$. By construction, $\varepsilon_{L}^{*}$ is a homomorphism of Boolean algebras. Thus, $\varepsilon_{L}$ is an isomorphism of measurable locales.

To show the naturality of $\varepsilon$, consider the following square for an arbitrary map of measurable locales $f: L \rightarrow L^{\prime}:$

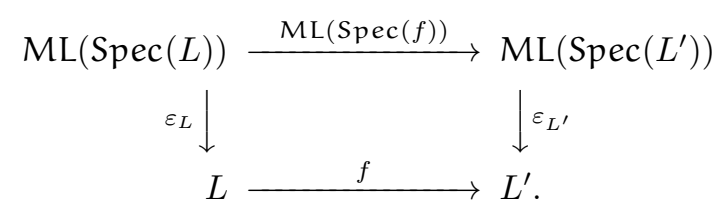

It suffices to show the commutativity of the induced diagram of inverse image maps:

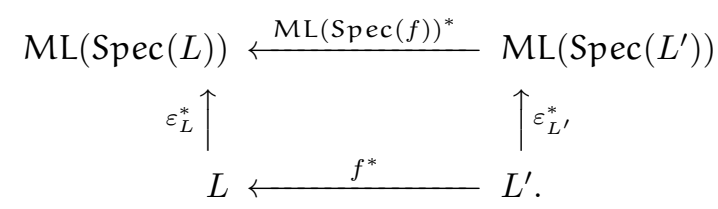

Pick an arbitrary element $m^{\prime} \in L^{\prime}$. The element $\varepsilon_{L}^{*}\left(f^{*}\left(m^{\prime}\right)\right)$ is the equivalence class of the clopen subset of $\operatorname{Spec}(L)$ corresponding to $f^{*} m^{\prime} \in L$. The element $\operatorname{ML}(\operatorname{Spec}(f))^{*}\left(\varepsilon_{L^{\prime}}^{*}\left(m^{\prime}\right)\right)$ is the equivalence class of the preimage under $\operatorname{Spec}(f)$ of the clopen subset of $\operatorname{Spec}\left(L^{\prime}\right)$ corresponding to $m^{\prime}$. Using the equality Sped $=\mathrm{TM} \circ \mathrm{Sp} \circ$ Ideal and the adjoint equivalence $\Omega-\mathrm{Sp}$ of Proposition 2.72, we can equivalently describe this element as the equivalence class of the clopen subset of $\operatorname{Spec}(L)$ corresponding to the ideal $\operatorname{Id}\left(f^{*}\right)\left(m^{\prime}\right)$, i.e., the ideal of $L$ generated by the image of the principal ideal of $m^{\prime}$ under $f^{*}$. The latter ideal is precisely the principal ideal of $f^{*} m^{\prime}$. Thus, the diagram commutes.

The following Proposition 5.16 is the technical heart of the paper. It makes essential use of both strict localizability and compactness.

The map $\eta$ in Proposition 5.16 is supplied by the von Neumann-Maharam lifting theorem. See Maharam [1958] for the $\sigma$-finite case and Fremlin [2002.d, Corollary 341Q] for the strictly localizable case. The strict localizability of $(X, M, N)$ is crucial at this point, since Fremlin [2002.d, Proposition 341M] shows that the existence of the map $\eta$ implies strict localizability.

The construction of the inverse map $\psi$ in Proposition 5.16 is due to von Neumann 1932, C. Ionescu Tulcea [1965], Vesterstrøm-Wils 1969], Edgar [1976, Proposition 3.4], and Graf [1980, Theorem 1]. For an exposition, see Fremlin [2002.d, Theorem 343B(iv)]. The compactness of $(X, M, N)$ is crucial for these results to be applicable, since Fremlin [1999, §3, Theorem] and Rinkewitz [2002.a, Theorem 2.4] show that the existence of the map $\psi$ implies compactness.

Proposition 5.16. For any $(X, M, N) \in$ CSLEMS there is an isomorphism

$$
\left.\eta_{(X, M, N)}:(X, M, N) \rightarrow \operatorname{Sped} M \square(X, M, N)\right),
$$

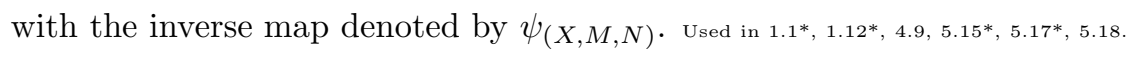

Proof. Given $E \in M$, denote by $E^{\star}$ the clopen subset of $\operatorname{Spec}(\operatorname{ML}(X, M, N))$ corresponding to the element $[E] \in \mathrm{ML}(X, M, N)=M / N$. Since $(X, M, N)$ is strictly localizable, by Fremlin 2002.d, Corollary 341Q], if $X \notin N$, there is a map of sets

$$
\eta: X \rightarrow \operatorname{Spec}(\operatorname{ML}(X, M, N))
$$

such that $E \oplus \eta^{*}\left(E^{\star}\right) \in N$ for all $E \in M$ and $\eta^{*} F \in N$ for all negligible subsets $F \subset \operatorname{Spec}(M \square(X, M, N))$. (Choose an arbitrary positive faithful semifinite measure $\mu$ on $(X, M, N)$ to satisfy the conditions of the cited result.) We recall the construction of $\eta$ : if $X \notin N$, then by the von Neumann-Maharam lifting theorem (Fremlin [2002.d, Theorem 341K]) the quotient homomorphism $M \rightarrow M / N$ has a section $\theta: M / N \rightarrow M$, which is a homomorphism of Boolean algebras. Then the point $\eta(x) \in \operatorname{Spec} M \square(X, M, N))$ is defined as the homomorphism of Boolean algebras $M / N \rightarrow \mathbf{Z} / 2$ that sends $m \in M / N$ to 1 if $x \in \theta(m)$ and 0 otherwise. (Recall that points of $\operatorname{Spec}(A)$ are precisely homomorphisms $A \rightarrow \mathbf{Z} / 2$.) By construction, $\theta([E])=\eta^{*} E^{\star}$ for all $E \in M$, in particular, $\eta^{*} E^{\star} \in M$ for all $E \in M$. 
Any measurable subset of $\operatorname{Spec}(\mathrm{ML}(X, M, N))$ is by definition the symmetric difference of $E^{\star}$ for some $E \in M$ and a meager subset, and preimages of meager subsets under $\eta$ belong to $N$ (Fremlin 2002.d, Proposition $341 \mathrm{P}(\mathrm{b})])$, so this implies that $\eta:(X, M, N) \rightarrow \operatorname{Spec} \mathrm{ML}(X, M, N))$ is a map of enhanced measurable spaces.

If $X \in N$, then $M / N=\{0\}$, and hence $\operatorname{Spec}(\operatorname{ML}(X, M, N))=\emptyset$, so we take the (unique) map of sets $\eta: \emptyset \rightarrow \operatorname{Spec}(\mathrm{ML}(X, M, N))$, which yields a map of enhanced measurable spaces $\eta:(X, M, N) \rightarrow$ $\operatorname{Sped}(\mathbb{M L}(X, M, N))$ with pdom $\eta=\emptyset \subset X$. It is precisely at this point that it is crucial to allow the underlying maps of sets of morphisms of enhanced measurable spaces to have a point-set domain different from $X$.

Since $(X, M, N)$ is compact, by Fremlin [2002.d, Theorem 343B(iv)], we have a map of sets

$$
\left.\psi_{(X, M, N)}: \operatorname{Sped} \operatorname{ML}(X, M, N)\right) \rightarrow X
$$

such that $\psi^{*} E \oplus E^{\star}$ is negligible in the enhanced measurable space $\operatorname{sped}(M \square(X, M, N))$ for all $E \in M$. The map $\psi$ is constructed by setting $\psi(z) \in X$ to any element in the (nonempty) intersection of all sets $K \in K_{X}$ such that $z \in K^{\star}$. Here $K_{X}$ is the compact class that witnesses the compactness of $(X, M, N)$. If there are no such $K$, then $\psi(z)$ can be set to any point in $X$. The properties of $\psi$ cited above imply that

$$
\psi: \operatorname{Sped} M(X, M, N)) \rightarrow(X, M, N)
$$

is a map of enhanced measurable spaces because $\psi^{*} n \oplus n^{\star}=\psi^{*} n \oplus \emptyset=\psi^{*} n \in N \operatorname{spec}(\overline{\mathrm{ML}}(X, M, N))$ for any $n \in N$ and $\psi^{*} m \oplus m \in M$ Spec $\left.\mid M L(X, M, N)\right)$ for any $m \in M$, so $\psi^{*} m$ is the symmetric difference of $m$ and some negligible subset of $\operatorname{Spec}(M \mathrm{ML}(X, M, N))$ and therefore is measurable.

For any $m \in M$ we have

$$
\eta^{*} \psi^{*} m \oplus m=\eta^{*}\left(m^{\star} \oplus n\right) \oplus m=\eta^{*} m^{\star} \oplus m \oplus \eta^{*} n=n^{\prime} \oplus \eta^{*} n \in N,
$$

for some negligible subset $n \subset \operatorname{Sped} \mathrm{ML}(X, M, N))$ and some $n^{\prime} \in N$. Thus, $\psi \circ \eta \approx \operatorname{id}_{(X, M, N)}$. Likewise, for any measurable subset of $\operatorname{Spec}(M \mathrm{ML}(X, M, N))$, which can be assumed to be a clopen subset of the form $E^{\star}$, the subset $\psi^{*} \eta^{*} E^{\star}=\psi^{*}\left(E \oplus n^{\prime}\right)=\psi^{*} E \oplus \psi^{*} n^{\prime}=E^{\star} \oplus n \oplus \psi^{*} n^{\prime}$ is a negligible subset of $\operatorname{Spec}(\mathbb{M} \mathrm{L}(X, M, N))$, for some $n^{\prime} \in N$ and some negligible subset $n \subset \operatorname{Spec}(M L(X, M, N))$. Thus, $\eta \circ \psi \approx \mathrm{id} \operatorname{Sped}(M L(X, M, N))$. Hence, $\eta$ and $\psi$ are mutually inverse and both are isomorphisms.

Proposition 5.17. There is a natural isomorphism

$$
\eta: \text { id CSLEMS } \rightarrow \text { SpeC } \mathrm{ML}
$$

of functors CSLEMS $\rightarrow$ CSLEMS.

Proof. It remains to show that the maps $\eta$ constructed in Proposition 5.16 are natural. The square

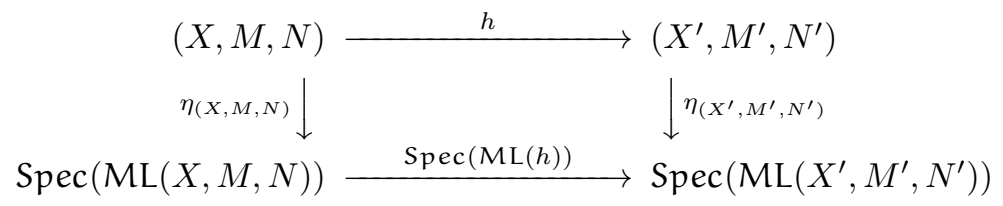

commutes if and only if its image under ML (depicted by the upper square below) does:

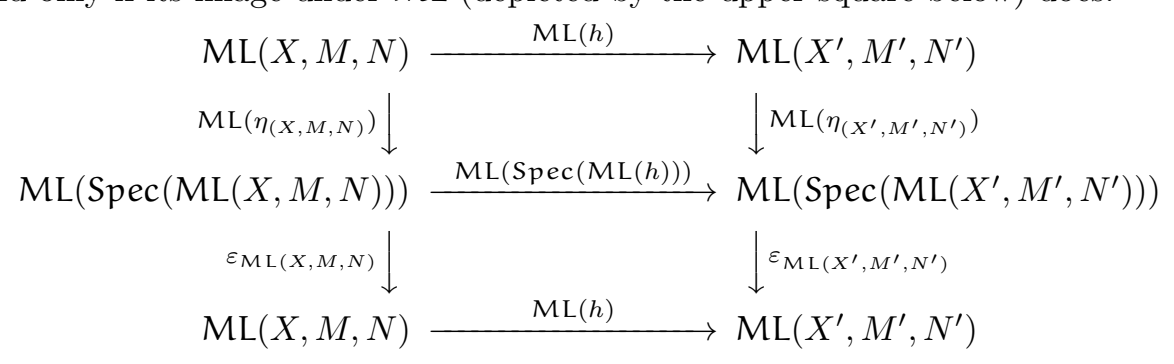

By construction, we have $M \mathcal{M L}\left(\eta_{(X, M, N)}\right)=\varepsilon \sqrt{M L}(X, M, N)$ and likewise for the bottom right map. Thus, the vertical compositions are identities, which completes the proof since the top and bottom maps must be the same. 
Remark 5.18. The functor Sped lands inside CSLEMS and by Lemma 4.63 the full subcategory CSLEMS $\subset$ LDEMS is closed under isomorphisms. Thus, $(X, M, N)$ must be compact and strictly localizable for Proposition 5.16 to hold, assuming $(X, M, N)$ is locally determined (Definition 4.50). By Fremlin [2001, §216E], there are localizable enhanced measurable spaces that are not strictly localizable, so the category LEMS is not equivalent to MLod By Remark 5.12, there is a morphism in LEMS whose image under WeakML does not preserve suprema. If we discard such morphisms and pass to the nonfull subcategory LEMSc with the same objects and morphisms whose image under WeakML preserves suprema, the functor WeakML restricts to a full functor ML: LEMS $\rightarrow$ MLog. If we now further perform a Gabriel-Zisman localization of LEMS with respect to the morphisms $f$ such that ML $(f)$ is an isomorphism, we obtain a new category LEMSd together with the induced localization functor LEMSd $\rightarrow$ MLod. The category CSLEMS is a full subcategory of LEMSd. The functor LEMSd $\rightarrow$ MLod is essentially surjective and full because its restriction to CSLEMS is essentially surjective and full. It is faithful by definition of weak equality almost everywhere. Thus, LEMSd and MLod are equivalent categories. However, morphisms in CSLEMS (or in EMS) admit a point-set description as equivalence classes of certain maps of sets, whereas morphisms in LEMSd' do not

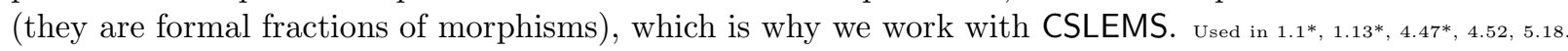

Theorem 5.19. The functors

$$
\text { ML: CSLEMS } \rightarrow \text { MLOC }
$$

and

$$
\text { Spec: MLod } \rightarrow \text { CSLEMS }
$$

together with natural isomorphisms

$$
\eta: \text { id } \rightarrow \text { Spec } \circ M L
$$

and

$$
\varepsilon: M L \circ \text { Spec } \rightarrow \text { id }
$$

form an adjoint equivalence of categories. Used in 1.1.

Proof. It remains to show that the exhibited equivalence is an adjoint equivalence. The two triangle identities imply one another, so it suffices to establish just one of them. To show that the composition

$$
\left.\left.\mathrm{ML}(X, M, N) \stackrel{\mathrm{ML}\left(\eta_{(X, M, N)}\right)}{\longrightarrow} \mathrm{ML} \operatorname{Spec} M \mathrm{ML}(X, M, N)\right)\right) \stackrel{\mathrm{ML}(X, M, N)}{\longrightarrow} \mathrm{ML}(X, M, N)
$$

equals identity it suffices to observe that the map $\varepsilon \overline{M L}(X, M, N)$ sends any $m \in \mathbb{M L}(X, M, N)=M / N$ to the equivalence class of the clopen subset $m^{\star}$ of $\left.\operatorname{Spec} M L(X, M, N)\right)$. Then the map $\operatorname{ML}\left(\eta_{(X, M, N)}\right)$ sends this class to the equivalence class of $\eta_{(X, M, N)}^{*} m^{\star}$, which differs from $m$ by an element of $N$, and hence yields the same element of $M / N$.

\section{References}

All bibliographic entries are equipped with hyperlinked back references. An asterisk $\left(^{*}\right)$ after a back reference indicates that the corresponding entry is referenced after the given numbered statement.

[1932] John von Neumann. Einige Sätze über messbare Abbildungen. Annals of Mathematics 33:3 (1932), 574-586. doi:10.2307/1968536. 1.0*, 5.15*.

[1936] Marshall H. Stone. The theory of representations for Boolean algebras. Transactions of the American Mathematical Society 40:1 (1936), 37-111. doi:10.1090/s0002-9947-1936-1501865-8. $2.0^{*}, 2.33^{*}$.

[1937.a] Marshall H. Stone. Applications of the theory of Boolean rings to general topology. Transactions of the American Mathematical Society 41:3 (1937), 375-481. doi:10.1090/s0002-9947-1937-1501905-7 1.0*, 2.0*, 2.33*.

[1937.b] Marshall H. Stone. Algebraic characterizations of special Boolean rings. Fundamenta Mathematicae 29:1 (1937), 223-303. https://eudml.org/doc/212943 2.0* 2.42*. 
[1938] Marshall H. Stone. Topological representation of distributive lattices and Brouwerian logics. Časopis pro pěstování matematiky a fysiky 67:1 (1938), 1-25.

https://hdl.handle.net/10338.dmlcz/124080 2.0*

[1941] Israel Gelfand. Normierte Ringe. Recueil Mathématique 9(51):1 (1941), 3-24.

http://mi.mathnet.ru/msb6046. 1.0*.

[1942] Paul R. Halmos, John von Neumann. Operator methods in classical mechanics, II. Annals of Mathematics 43:2 (1942), 332-350. doi:10.2307/1968872 1.2*.

[1947] Lynn H. Loomis. On the representation of $\sigma$-complete Boolean algebras. Bulletin of the American Mathematical Society 53:8 (1947), 757-760. doi:10.1090/s0002-9904-1947-08866-2 $1.0^{*}, 5.1$.

[1948] Roman Sikorski. On the representation of Boolean algebras as fields of sets. Fundamenta Mathematicae 35 (1948), 247-258. doi:10.4064/fm-35-1-247-258, 1.0*, 5.1*.

[1951.a] Jacques Dixmier. Sur certains espaces considérés par M. H. Stone. Summa Brasiliensis Mathematicae 2 (1951), 151-182. https://dmitripavlov.org/scans/dixmier.pdf. 1.0*, 2.51*, 2.64*, $2.65^{*}, 2.67^{*}, 2.70^{*}, 4.36$.

[1951.b] Irving E. Segal. Equivalences of measure spaces. American Journal of Mathematics 73:2 (1951), 275-313. doi:10.2307/2372178 4.33*, 4.34*, 4.36.

[1951.c] Irving E. Segal. Decompositions of operator algebras. II: Multiplicity theory. Memoirs of the American Mathematical Society 9 (1951). doi:10.1090/memo/0009, 1.3*, 3.0*, 3.19*.

[1953] Edward Marczewski. On compact measures. Fundamenta Mathematicae 40:1 (1953), 113-124. doi:10.4064/fm-40-1-113-124, 4.56*.

[1956] Shôichirô Sakai. A characterization of $W^{*}$-algebras. Pacific Journal of Mathematics 6:4 (1956), 763-773. doi:10.2140/pjm.1956.6.763. 3.4.

[1958] Dorothy Maharam. On a theorem of von Neumann. Proceedings of the American Mathematical Society 9:6 (1958), 987-987. doi:10.1090/s0002-9939-1958-0105479-6 1.0*, 5.15*.

[1965] Cassius Ionescu Tulcea. Sur certains endomorphismes de $\mathrm{L}_{\mathrm{C}}^{\infty}(Z, \mu)$. Comptes Rendus Hebdomadaires des Séances de l'Académie des Sciences 261 (1965), 4961-4963. https://gallica.bnf.fr/ark:/12148/bpt6k4027h/ 1.0*, 5.15*.

[1966] Johann Pfanzagl, W. Pierlo. Compact Systems of Sets. Lecture Notes in Mathematics 16 (1966). doi:10.1007/BFb0078990 4.56*.

[1969] Jørgen Vesterstrøm, Wilbert Wils. On point realizations of $L^{\infty}$-endomorphisms. Mathematica Scandinavica 25 (1969), 178-180. doi:10.7146/math.scand.a-10954 1.0*, 5.15*.

[1971.a] Joan W. Negrepontis. Duality in analysis from the point of view of triples. Journal of Algebra 19:2 (1971), 228-253. doi:10.1016/0021-8693(71)90105-0. 1.0*.

[1971.b] Shôichirô Sakai. $C^{*}$-Algebras and $W^{*}$-Algebras. Ergebnisse der Mathematik und ihrer Grenzgebiete 60 (1971). doi:10.1007/978-3-642-61993-9. 1.4*, 3.1*, 3.4, 3.7, 3.9*.

[1976] Gerald A. Edgar. Measurable weak sections. Illinois Journal of Mathematics 20:4 (1976), 630-646. doi:10.1215/ijm/1256049654 1.0* 5.15*.

[1980] Siegfried Graf. Induced $\sigma$-homomorphisms and a parametrization of measurable sections via extremal preimage measures. Mathematische Annalen 247:1 (1980), 67-80. doi:10.1007/bf01359867 1.0*, 5.15*.

[1982] Peter T. Johnstone. Stone spaces. Cambridge Studies in Advanced Mathematics 3 (1982). https://b-ok.cc/md5/CFB5295DDC01BCE9CAE1256930A62B5A 1.4*, 2.23*, 2.26*, 2.29, 2.37, $2.48^{*}$.

[1983] Peter T. Johnstone. The point of pointless topology. Bulletin of the American Mathematical Society 8:1 (1983), 41-53. doi:10.1090/s0273-0979-1983-15080-2. 2.9*.

[1991] Peter T. Johnstone. The art of pointless thinking: a student's guide to the category of locales. Category theory at work. Research and Exposition in Mathematics 18 (1991), 85-107. http://www.heldermann.de/R\&E/RAE18/ctw06.pdf 2.9*.

[1992] Michael Albert Wendt. On measurably indexed families of Hilbert spaces. Ph.D. dissertation, Dalhousie University, 1992. https://hdl.handle.net/10222/55327. 4.6*. 
[1993] David H. Fremlin. Real-valued-measurable cardinals. Set theory of the reals. Israel Mathematical Conference Proceedings 6 (1993), 151-304.

https://www1.essex.ac.uk/maths/people/fremlin/rvmc.pdf 5.12.

[1994.a] Francis Borceux. Handbook of Categorical Algebra 3. Categories of Sheaves. Encyclopedia of Mathematics and its Applications 52 (1994). doi:10.1017/CBO9780511525872 2.1*.

[1994.b] Michael A. Wendt. The category of disintegration. Cahiers de Topologie et Géométrie Différentielle Catégoriques 35:4 (1994), 291-308. https://eudml.org/doc/91550. 4.6*.

[1996] Michael A. Wendt. Measurable Hilbert sheaves. Journal of the Australian Mathematical Society (Series A) 61:2 (1996), 189-215. doi:10.1017/s1446788700000197, 4.6*.

[1998] Michael A. Wendt. Change of base for measure spaces. Journal of Pure and Applied Algebra 128 (1998), 185-212. doi:10.1016/s0022-4049(97)00048-0. 4.6*.

[1999] David H. Fremlin. Compact measure spaces. Mathematika 46:2 (1999), 331-336. doi:10.1112/S0025579300007798. 4.62*, 5.15*.

[2001] David H. Fremlin. Measure theory. Volume 2. Torres Fremlin, Colchester, 2001. https://www1.essex.ac.uk/maths/people/fremlin/mt.htm 2.59*, 4.42*, 4.43, 4.49, 4.51, 4.52, $4.53 *, 5.18$.

[2002.a] Werner Rinkewitz. Compact measures and measurable weak sections. Mathematica Scandinavica 91:1 (2002), 150-160. doi:10.7146/math.scand.a-14383 5.15*.

[2002.b] David A. Edwards. On function lattices and commutative von Neumann algebras. The Quarterly Journal of Mathematics 53:1 (2002), 19-29. doi:10.1093/qjmath/53.1.19. 2.46*.

[2002.c] Mauricio Alvarez-Manilla. Extension of valuations on locally compact sober spaces. Topology and its Applications 124 (2002), 397-433. doi:10.1016/s0166-8641(01)00249-8, 2.70.

[2002.d] David H. Fremlin. Measure theory. Volume 3. Torres Fremlin, Colchester, 2002. https://www1.essex.ac.uk/maths/people/fremlin/mt.htm 1.2, 1.4 *, 4.23, 4.44, 4.58, 4.62*, $5.15^{*}, 5.16^{*}$.

[2003] David H. Fremlin. Measure theory. Volume 4. Torres Fremlin, Colchester, 2003. https://www1.essex.ac.uk/maths/people/fremlin/mt.htm 1.4*, 1.13*, 2.64, 4.60, 4.65*.

[2005] Hans F. de Groote. Observables I: Stone spectra. arXiv:math-ph/0509020v1. 3.12.

[2006] Matthew Jackson. A sheaf theoretic approach to measure theory. Ph.D. dissertation, University of Pittsburgh, 2006. ISBN: 978-0542-74794-6.

https://d-scholarship.pitt.edu/7348/1/Matthew_Jackson_Thesis_2006.pd丹 1.2 .

[2009] Dmitri Pavlov. Answer 3279 (revision 1) on MathOverflow. October 29, 2009. https://mathoverflow.net/revisions/3279/1. 1.13*.

[2010.a] Dmitri Pavlov. Answer 20820 (revision 1) on MathOverflow. April 9, 2010. https://mathoverflow.net/revisions/20820/1, 1.13*.

[2010.b] Dmitri Pavlov. Question 23480 (revision 1) on MathOverflow. May 4, 2010. https://mathoverflow.net/revisions/23408/1. 1.13*.

[2010.c] Dmitri Pavlov. Answer 49542 (revision 1) on MathOverflow. December 15, 2010. https://mathoverflow.net/revisions/49542/1, 1.2*, 1.13*.

[2011] Hans F. de Groote. Classical and Quantum Observables. Deep Beauty, Cambridge University Press (2011), 239-269. doi:10.1017/cbo9780511976971. 3.12.

[2012] Jorge Picado, Aleš Pultr. Frames and Locales. Topology without points. Frontiers in Mathematics (2012), Birkhäuser. doi:10.1007/978-3-0348-0154-6. 2.9*.

[2014] Simon Henry. Localic metric spaces and the localic Gelfand duality. Advances in Mathematics 294 (2016), 634-688. arXiv:1411.0898v1. doi:10.1016/j.aim.2016.03.006 1.12*. 